\title{
THE PLURIPOTENTIAL CAUCHY-DIRICHLET PROBLEM FOR COMPLEX MONGE-AMPĖRE FLOWS
}

\author{
VINCENT GUEDJ, CHINH H. LU, AND AHMED ZERIAHI
}

\begin{abstract}
We develop the first steps of a parabolic pluripotential theory in bounded strongly pseudo-convex domains of $\mathbb{C}^{n}$. We study certain degenerate parabolic complex Monge-Ampère equations, modelled on the Kähler-Ricci flow evolving on complex algebraic varieties with Kawamata log-terminal singularities.

Under natural assumptions on the Cauchy-Dirichlet boundary data, we show that the envelope of pluripotential subsolutions is semi-concave in time and continuous in space, and provides the unique pluripotential solution with such regularity.
\end{abstract}

\section{CONTEnTs}

Introduction $\quad 1$

1. Families of plurisubharmonic functions $\quad 7$

2. Parabolic Monge-Ampère operators 16

3. Boundary behavior of parabolic envelopes 22

4. Time regularity of parabolic envelopes 30

5. Space regularity of parabolic envelopes 38

6. Pluripotential solutions 43

References $\quad 54$

\section{INTRODUCTION}

The Ricci flow, first introduced by Hamilton [Ham82] is the equation

$$
\frac{\partial}{\partial t} g_{i j}=-2 R_{i j}
$$

evolving a Riemannian metric by its Ricci curvature. If the Ricci flow starts from a Kähler metric, the evolving metrics remain Kähler and the resulting PDE is called the Kähler-Ricci flow.

Date: October 5, 2018.

2010 Mathematics Subject Classification. 53C44, 32W20, 58J35.

Key words and phrases. Complex Monge-Ampère flow, pluripotential solution, Perron envelope, comparison principle.

The authors are partially supported by the ANR project GRACK. 
It is expected that the Kähler-Ricci flow can be used to give a geometric classification of complex algebraic and Kähler manifolds, and produce canonical metrics at the same time. Solving the Kähler-Ricci flow boils down to solving a parabolic scalar equation modeled on

$$
\operatorname{det}\left(\frac{\partial^{2} u_{t}}{\partial z_{j} \partial \bar{z}_{k}}(t, z)\right)=e^{\partial_{t} u_{t}(z)+H(t, z)+\lambda u_{t}(z)}
$$

where $t \mapsto u_{t}(z)=u(t, z)$ is a smooth family of strictly plurisubharmonic functions in $\mathbb{C}^{n}, \lambda \in \mathbb{R}$ and $g=e^{H}$ is a smooth and positive density.

It is important for geometric applications to study degenerate versions of these complex Monge-Ampère flows, where the functions $u_{t}$ are no longer smooth nor strictly plurisubharmonic, and the densities may vanish or blow up (see [SW13, CT15, ST17, EGZ18] and the references therein).

A viscosity approach has been developed recently in [EGZ15], following its elliptic counterpart [EGZ11, HL11, HL13]. While the viscosity theory is very robust, it requires the data to be continuous hence has a limited scope of applications. Several geometric situations encountered in the Minimal Model program (MMP) necessitate one to deal with Kawamata log-terminal (klt) singularities. The viscosity approach breaks down in these cases and a more flexible method is necessary.

There is a well established pluripotential theory of weak solutions to degenerate elliptic complex Monge-Ampère equations, following the pioneering work of Bedford-Taylor [BT76, BT82]. This theory allows to deal with $L^{p}$ densities as established in a corner stone result of Kołodziej [Kol98], which provides a great generalization of [Yau78].

No similar theory has ever been developed on the parabolic side. The purpose of this article, the first of a series on this subject, is to develop a pluripotential theory for degenerate complex Monge-Ampère flows. This article settles the foundational material for this theory and focuses on solving the Cauchy-Dirichlet problem in domains of $\mathbb{C}^{n}$.

We consider the following family of Monge-Ampère flows

$$
d t \wedge\left(d d^{c} u\right)^{n}=e^{\partial_{t} u+F(t, z, u)} g(z) d t \wedge d V,
$$

in $\left.\Omega_{T}:=\right] 0, T\left[\times \Omega\right.$, where $d V$ is the euclidean volume form on $\mathbb{C}^{n}$ and

- $T>0$ and $\Omega \Subset \mathbb{C}^{n}$ is a bounded strictly pseudoconvex domain;

- $F(t, z, r)$ is continuous in $[0, T[\times \Omega \times \mathbb{R}$, increasing in $r$, bounded in $[0, T[\times \Omega \times J$, for each $J \Subset \mathbb{R}$;

- $(t, r) \mapsto F(t, \cdot, r)$ is uniformly Lipschitz and semi-convex in $(t, r)$;

- $g \in L^{p}(\Omega), p>1$, and $g>0$ almost everywhere ;

- $u:[0, T[\times \Omega \rightarrow \mathbb{R}$ is the unknown function.

Here $d=\partial+\bar{\partial}$ and $d^{c}=i(\bar{\partial}-\partial) / 2$ so that $d d^{c}=i \partial \bar{\partial}$ and $\left(d d^{c} u\right)^{n}$ represents the determinant of the complex Hessian of $u$ in space (the complex Monge-Ampère operator) whenever $u$ is $\mathcal{C}^{2}$-smooth. 
For less regular functions $u$, the equation (CMAF) should be understood in the weak sense of pluripotential theory as we explain in Section 2.

We let $\mathcal{P}\left(\Omega_{T}\right)$ denote the set of parabolic potentials, i.e. those functions $u: \Omega_{T} \rightarrow\left[-\infty,+\infty\left[\right.\right.$ defined in $\left.\Omega_{T}=\right] 0, T[\times \Omega$ and satisfying the following conditions:

- for any $t \in] 0, T[, u(t, \cdot)$ is plurisubharmonic in $\Omega$;

- the family $\{u(\cdot, z) ; z \in \Omega\}$ is locally uniformly Lipschitz in $] 0, T[$.

We study in Section 1 basic properties of parabolic potentials. We show in Lemma 1.3 that if $u \in \mathcal{P}\left(\Omega_{T}\right)$ and is bounded from above in $\Omega_{T}$ then it can be uniquely extended as an upper-semicontinuous function in $[0, T[\times \Omega$ such that $u(0, \cdot)$ is plurisubharmonic in $\Omega$. We show that parabolic potentials satisfy approximate submean value inequalities (Lemma 1.6) and enjoy good compactness properties (Proposition 1.14).

We show in Section 2 that parabolic complex Monge-Ampère operators are well defined on $\mathcal{P}\left(\Omega_{T}\right) \cap L_{\text {loc }}^{\infty}\left(\Omega_{T}\right)$ and enjoy nice continuity properties, allowing to make sense of pluripotential sub/super/solutions to (CMAF) (see Definition 3.1). A crucial convergence property is obtained in Proposition 2.9 , under a semi-concavity assumption on the family of parabolic potentials.

A Cauchy-Dirichlet boundary data is a function $h$ defined on the parabolic boundary of $\Omega_{T}$ denoted by

$$
\partial_{0} \Omega_{T}:=([0, T[\times \partial \Omega) \cup(\{0\} \times \Omega),
$$

such that

- the restriction of $h$ on $[0, T[\times \partial \Omega$ is continuous;

- the family $\{h(\cdot, z) ; z \in \partial \Omega\}$ is locally uniformly Lipschitz in $] 0, T[$;

- $h$ satisfies the following compatibility condition : $\forall \zeta \in \partial \Omega$,

$$
h_{0}:=h(0, \cdot) \in \operatorname{PSH}(\Omega) \cap L^{\infty}(\Omega) \text { and } \lim _{\Omega \ni z \rightarrow \zeta} h(0, z)=h(0, \zeta) .
$$

The Cauchy-Dirichlet problem for the parabolic equation (CMAF) with Cauchy-Dirichlet boundary data $h$ consists in finding $u \in \mathcal{P}\left(\Omega_{T}\right) \cap L^{\infty}\left(\Omega_{T}\right)$ such that (CMAF) holds in the pluripotential sense in $\Omega_{T}$ and the following Cauchy-Dirichlet boundary conditions are satisfied :

$$
\begin{gathered}
\forall(\tau, \zeta) \in\left[0, T\left[\times \partial \Omega, \quad \lim _{\Omega_{T} \ni(t, z) \rightarrow(\tau, \zeta)} u(t, z)=h(\tau, \zeta) .\right.\right. \\
\lim _{t \rightarrow 0^{+}} u_{t}=h_{0} \text { in } L^{1}(\Omega) .
\end{gathered}
$$

In this case we say that $u$ is a solution to the Cauchy-Dirichlet problem for the equation (CMAF) with boundary values $h$.

Observe that a solution $u$ to the equation (CMAF) has plurisubharmonic slices in $\Omega$ and the Cauchy condition (0.3) implies by a classical result in pluripotential theory that $\left(\lim \sup _{t \rightarrow 0} u_{t}\right)^{*}=h_{0}^{*} \in \operatorname{PSH}(\Omega)$, hence $h_{0}=$ 
$h_{0}^{*} \in \operatorname{PSH}(\Omega)$. This observation shows that the Cauchy data $h_{0}$ must be plurisubharmonic as it is required in the compatibility condition (0.1).

For a solution to the Cauchy-Dirichlet problem for the equation (CMAF), the Cauchy condition (0.3) implies that

$$
\forall z \in \Omega, \lim _{t \rightarrow 0^{+}} u_{t}(z)=h_{0}(z) .
$$

It is possible to consider less regular initial Cauchy data $h(0, \cdot)$ (see [Do17, Do16]), but we will not pursue this here.

We try and construct a solution to the Cauchy-Dirichlet problem by the Perron method, considering the upper envelope $U$ of pluripotential subsolutions.

The technical core of the paper lies in Section 3 and Section 4. In Section 3 we construct subbarriers and controls from above to ensure that $U$ has the right boundary values (see Theorem 3.12). In Section 4 we prove that the Perron envelope of subsolutions is locally uniformly Lipschitz and semiconcave in time.

Theorem A. Assume $h$ is a Cauchy-Dirichlet boundary data in $\Omega_{T}$ such that for all $0<S<T$, and for all $(t, z) \in] 0, S] \times \partial \Omega$,

$$
t\left|\partial_{t} h(t, z)\right| \leq C(S) \quad \text { and } \quad t^{2} \partial_{t}^{2} h(t, z) \leq C(S),
$$

Then the envelope $U=U_{h, g, F}$ is locally uniformly Lipschitz and locally uniformly semi-concave in $t \in] 0, T[$. Moreover, $U$ satisfies the CauchyDirichlet boundary conditions (0.2), (0.3).

Here $C(S)$ is a positive constant depending on $S$ which may blow up as $S \rightarrow T$. The proof of Theorem A, which shows in particular that $U$ satisfies $(\dagger)$, is given in Theorem 4.2, Theorem 4.6 and Theorem 4.7. The Lipschitz and semi-concave constants of $U$ depend explicitly on $C(S)$.

We prove in Theorem 5.1 that the envelope $U$ is moreover (Lipschitz) continuous in space if so are the data $\left(h_{0}, \log g, F\right)$.

Focusing for a while on the case of the unit ball with regular boundary data, we obtain the following parabolic analogue of Bedford and Taylor's celebrated result [BT76] :

Theorem B. Assume $\Omega=\mathbb{B}$ is the unit ball in $\mathbb{C}^{n}$ and

- $G:=\log g$ is $\mathcal{C}^{1,1}$ in $\overline{\mathbb{B}}$;

- $h$ is uniformly Lipschitz in $t \in\left[0, T\left[\right.\right.$, satisfies $\partial_{t}^{2} h(t, z) \leq C / t^{2}$, $z \in \partial \mathbb{B}$, and $h$ is uniformly $\mathcal{C}^{1,1}$ in $z \in \overline{\mathbb{B}}$;

- $F$ is Lipschitz and semi-convex in $[0, T[\times \overline{\mathbb{B}} \times J$, for each $J \Subset \mathbb{R}$.

Then the upper envelope $U:=U_{h, g, F}$ is locally uniformly $\mathcal{C}^{1,1}$ in $z$ and locally uniformly Lipschitz in $t \in] 0, T\left[\right.$. For almost any $(t, z) \in \mathbb{B}_{T}$, we have

$$
\operatorname{det}\left(\partial_{j} \bar{\partial}_{k} U(t, z)\right)=e^{\partial_{t} U(t, z)+F(t, z, U(t, z))} g(z) .
$$

In particular $U$ is a pluripotential solution to the Cauchy-Dirichlet problem for the parabolic equation (CMAF) with boundary values $h$. 
This result is obtained as a combination of Theorem 5.3 and Theorem 6.1. Using an approximation and balayage process we then treat the case of more general domains $\Omega$ with less regular boundary data, obtaining the following solution to our original problem :

Theorem C. Assume $h$ is a Cauchy-Dirichlet boundary data in $\Omega_{T}$ such that for all $0<S<T$, and for all $(t, z) \in] 0, S] \times \partial \Omega$,

$$
t\left|\partial_{t} h(t, z)\right| \leq C(S) \text { and } t^{2} \partial_{t}^{2} h(t, z) \leq C(S),
$$

The envelope of all subsolutions to (CMAF) with Cauchy-Dirichlet boundary data $h$ is a pluripotential solution to this Cauchy-Dirichlet problem.

The proof of this fundamental result is given in Theorem 6.4. We eventually establish a comparison principle, which shows that $U_{h, g, F}$ is unique:

Theorem D. Same assumptions as in Theorem A. Let $\Phi$ be a bounded pluripotential subsolution to (CMAF) with boundary values $h_{\Phi}$. Let $\Psi$ be a bounded pluripotential supersolution with boundary values $h_{\Psi}$, such that $\Psi$ is locally uniformly semi-concave in $t \in] 0, T\left[\right.$ and $h_{\Phi}$ satisfies $(\dagger)$. Then

$$
h_{\Psi} \geq h_{\Phi} \text { on } \partial_{0} \Omega_{T} \Longrightarrow \Phi \leq \Psi \text { in } \Omega_{T} .
$$

In particular, there is a unique pluripotential solution to the CauchyDirichlet problem for (CMAF) with boundary data $h$, which is locally uniformly semi-concave in $t$.

The proof of Theorem D is given in Section 6.3; it uses some ideas from [GLZ18, DL17]. When all the data $(h, F, g, u)$ are continuous, one can show that the solution $U$ coincides with the viscosity solution constructed in [EGZ15]. We refer the reader to [GLZ3] for a detailed comparison of viscosity and pluripotential concepts.

\section{Notations and assumptions on the data}

We finish this introduction by fixing some notations that will be used throughout the paper.

The domain. In the whole article we let $d V$ denote the euclidean volume form in $\mathbb{C}^{n}$ and $\Omega \Subset \mathbb{C}^{n}$ be a strictly pseudoconvex domain : there exists a smooth function $\rho$ in a neighborhood $V$ of $\bar{\Omega}$ such that

$$
\Omega=\{z \in V ; \rho(z)<0\},
$$

where $\partial_{z} \rho \neq 0$ on $\partial \Omega$ and $\rho$ is strictly plurisubharmonic in $V$. We set $\left.\Omega_{T}:=\right] 0, T[\times \Omega$ with $T>0$. Most of the time we will assume that $T<+\infty$.

Recall that if a function $u: \Omega \rightarrow[-\infty,+\infty[$ is plurisubharmonic, then $d d^{c} u \geq 0$ is a positive current on $\Omega$. Here $d=\partial+\bar{\partial}$ and $d^{c}=(i / 2)(\bar{\partial}-\partial)$ are both real operators so that $d d^{c}=i \partial \bar{\partial}$.

We let $\mathbb{B}$ denote the euclidean unit ball in $\mathbb{C}^{n}$ and $\lambda_{\mathbb{B}}$ denote the normalized Lebesgue measure on $\mathbb{B}$. 
The function $F$. We assume that $F:[0, T[\times \Omega \times \mathbb{R} \rightarrow \mathbb{R}$ is continuous and

- bounded in $[0, T[\times \Omega \times J$ for each $0<S<T, J \Subset \mathbb{R}$;

- increasing in $r: r \mapsto F(t, x, r)$ is increasing for all $(t, x) \in \Omega_{T}$ fixed;

- locally uniformly Lipschitz in $(t, r)$ : for each compact $J \Subset \mathbb{R}$ and each $0<S<T$ there exists a constant $\kappa=\kappa(S, J)>0$ such that for all $t, \tau \in[0, S], z \in \Omega, r, r^{\prime} \in J$,

$$
\left|F(t, z, r)-F\left(\tau, z, r^{\prime}\right)\right| \leq \kappa\left(|t-\tau|+\left|r-r^{\prime}\right|\right) ;
$$

- locally uniformly semi-convex in $(t, r)$ : for each compact subset $[0, S] \times J \Subset[0, T[\times \mathbb{R}$ there exists a constant $C=C(S, J)>0$ such that, for any $z \in \Omega$, the function

$$
(t, r) \mapsto F(t, z, r)+C\left(t^{2}+r^{2}\right) \text { is convex in }[0, S] \times J .
$$

The density $g$. We assume that

- $0 \leq g \in L^{p}(\Omega)$ for some $p>1$ that is fixed thoughout the paper ;

- the set $\{z \in \Omega ; g(z)=0\}$ has Lebesgue measure zero.

Boundary data $h$. We assume throughout the article that

- $h: \partial_{0} \Omega_{T} \rightarrow \mathbb{R}$ is bounded, upper semi-continuous on $\partial_{0} \Omega_{T}$;

- the restriction of $h$ on $[0, T[\times \partial \Omega$ is continuous;

- $t \mapsto h(t, z)$ is locally uniformly Lipschitz in $] 0, T[$ : for all $0<S<T$ there is $C(S)>0$ such that for all $(t, z) \in] 0, S] \times \partial \Omega$,

$$
t\left|\partial_{t} h(t, z)\right| \leq C(S)
$$

- $h(0, \cdot)$ is bounded, plurisubharmonic in $\Omega$, and satisfies

$$
\lim _{\Omega \ni z \rightarrow \zeta} h(0, z)=h(0, \zeta), \forall \zeta \in \partial \Omega .
$$

We eventually also assume that $t \mapsto h(t, z)$ is locally uniformly semiconcave in $] 0, T[$ : for all $0<S<T$ there is $C(S)>0$ such that

$$
t^{2} \partial_{t}^{2} h(t, z) \leq C(S), \forall(t, z) \in[0, S] \times \partial \Omega .
$$

The constants. We fix once and for all various uniform constants:

$$
M_{h}:=\sup _{\partial_{0} \Omega_{T}}|h|, M_{F}:=\sup _{\Omega_{T}} F\left(\cdot, \cdot, M_{h}\right) .
$$

We fix a plurisubharmonic function $\rho$ in $\Omega$, continuous in $\bar{\Omega}$ so that

$$
\left(d d^{c} \rho\right)^{n}=g d V, \quad \rho=0 \text { in } \partial \Omega,
$$

in the weak sense in $\Omega$. Such a function exists by [Koł95, Koł98] and there is moreover a uniform a priori bound on $\rho$,

$$
\|\rho\|_{L^{\infty}(\Omega)} \leq c_{n}\|f\|_{L^{p}(\Omega)}^{1 / n},
$$

where $c_{n}>0$ is a uniform constant depending on $n, \Omega$. 


\section{FAMILIES OF PLURISUBHARMONIC FUNCTIONS}

Parabolic potentials form the basic objects of our study. They can be seen as weakly regular family of plurisubharmonic functions. In this section we define them and establish their first properties.

\subsection{Basic properties.}

1.1.1. Parabolic potentials. We start with some basic definitions which will be used throughout all the paper.

Definition 1.1. Let $\left.u: \Omega_{T}:=\right] 0, T[\times \Omega \longrightarrow[-\infty,+\infty[$ be a given function.

We say that the family $\{u(\cdot, z) ; z \in \Omega\}$ is locally uniformly Lipschitz in ] $0, T[$ if for any subinterval $J \Subset] 0, T$ [ there exists a constant $\kappa:=\kappa_{J}(u)>0$ such that

$$
u(t, z) \leq u(s, z)+\kappa|t-s|, \text { for all } s, t \in J \text { and } z \in \Omega .
$$

Definition 1.2. The set of parabolic potentials $\mathcal{P}\left(\Omega_{T}\right)$ is the set of functions $\left.u: \Omega_{T}:=\right] 0, T[\times \Omega \longrightarrow[-\infty,+\infty[$ such that

- for all $t \in] 0, T\left[\right.$, the slice $u_{t}: z \mapsto u(t, z)$ is plurisubharmonic in $\Omega$;

- the family $\{u(\cdot, z) ; z \in \Omega\}$ is locally uniformly Lipschitz in $] 0, T[$.

$\operatorname{PSH}(\Omega)$ embeds in $\mathcal{P}\left(\Omega_{T}\right)$ as the class of time independent potentials. Basic operations on plurisubharmonic functions extend naturally to parabolic potentials:

- if $u, v \in \mathcal{P}\left(\Omega_{T}\right)$ then $u+v \in \mathcal{P}\left(\Omega_{T}\right)$ and $\max (u, v) \in \mathcal{P}\left(\Omega_{T}\right)$;

- if $u \in \mathcal{P}\left(\Omega_{T}\right)$ and $\lambda \geq 0$ then $\lambda u$ is also a parabolic potential.

It follows from the next Lemma that parabolic potentials extend naturally as upper semi-continous functions in $[0, T[\times \Omega$.

Lemma 1.3. Let $u:] 0, T[\times \Omega \longrightarrow[-\infty,+\infty[$ be a function bounded from above and satisfying the following conditions :

(i) for any $t \in] 0, T\left[\right.$ the function $u_{t}:=u(t, \cdot)$ is plurisubharmonic in $\Omega$;

(ii) for all $z \in \Omega$ the function $u(\cdot, z)$ is upper semicontinuous in $] 0, T[$.

For $z \in \Omega$ we set

$$
u_{0}(z):=\left(\limsup _{t \rightarrow 0^{+}} u_{t}\right)^{*}(z)=\limsup _{\zeta \rightarrow z}\left(\limsup _{t \rightarrow 0^{+}} u_{t}(\zeta)\right) .
$$

Then $u_{0}$ is plurisubharmonic in $\Omega$ and the extension $u:[0, T[\times \Omega \rightarrow$ $[-\infty,+\infty[$ is upper semicontinuous in $[0, T[\times \Omega$.

Proof. Since $u$ is bounded from above we can assume that $u \leq 0$. Fix $\left(t_{0}, z_{0}\right) \in \Omega_{T}$ and let $r>0$ be such that $B\left(z_{0}, 2 r\right) \Subset \Omega$. Fix $\left.\delta \in\right] 0, r[$.

Since $u_{t} \leq 0$, by the submean value inequality for psh functions, we have for $\left|z-z_{0}\right| \leq \delta$ and $\left.t \in\right] 0, T[$,

$$
u(t, z) \leq \frac{1}{\operatorname{Vol}(B(z, r+\delta))} \int_{B\left(z_{0}, r\right)} u(t, \zeta) d V(\zeta)
$$


It thus follows from Fatou's Lemma and assumption (ii) that

$$
\limsup _{(t, z) \rightarrow\left(t_{0}, z_{0}\right)} u(t, z) \leq \frac{1}{\operatorname{Vol}\left(B\left(z_{0}, r+\delta\right)\right)} \int_{B\left(z_{0}, r\right)} u\left(t_{0}, \zeta\right) d V(\zeta) .
$$

Since $u\left(t_{0}, \cdot\right)$ is plurisubharmonic in $\Omega$, letting $\delta \rightarrow 0^{+}$and $r \rightarrow 0^{+}$we obtain

$$
\limsup _{(t, z) \rightarrow\left(t_{0}, z_{0}\right)} u(t, z) \leq u\left(t_{0}, z_{0}\right),
$$

which proves that $u$ is upper semi-continuous at $\left(t_{0}, z_{0}\right)$.

Now if $t_{0}=0$, since $\left\{u_{t} ; t \in\right] 0, T[\}$ is a family of plurisubharmonic functions in $\Omega$ which is uniformly bounded from above, it follows that $u_{0}$ is plurisubharmonic in $\Omega$. Then by (1.2),

$$
\limsup _{(t, z) \rightarrow\left(0, z_{0}\right)} u(t, z) \leq \frac{1}{\operatorname{Vol}\left(B\left(z_{0}, r+\delta\right)\right)} \int_{B\left(z_{0}, r\right)} u_{0}(\zeta) d V(\zeta) .
$$

Letting $r \rightarrow 0^{+}$we obtain, by the plurisubharmonicity of $u_{0}$,

$$
\limsup _{(t, z) \rightarrow\left(0, z_{0}\right)} u(t, z) \leq u_{0}\left(z_{0}\right)=: u\left(0, z_{0}\right),
$$

which proves the semi-continuity of the extension at the point $\left(0, z_{0}\right)$.

The next result provides a parabolic analogue of a classical result of Lelong about negligible sets for plurisubharmonic functions; it will play an important role in section 3 .

Lemma 1.4. Let $\mathcal{U} \subset \mathcal{P}\left(\Omega_{T}\right)$ be a family of functions which is locally uniformly bounded from above. Assume $U:=\sup \{u ; u \in \mathcal{U}\}$ is locally uniformly Lipschitz in $t \in] 0, T[$. Then

- the upper semicontinuous regularization $U^{*}$ (in $\Omega_{T}$ ) belongs to $\mathcal{P}\left(\Omega_{T}\right)$;

- for any $t \in] 0, T\left[, U^{*}(t, \cdot)=\left(U_{t}\right)^{*}\right.$ in $\Omega$ and the exceptional set

$$
E(U):=\left\{(t, z) \in \Omega_{T} ; \quad U(t, z)<U^{*}(t, z)\right\}
$$

has zero $(2 n+1)$-dimensional Lebesgue measure in $\Omega_{T} \subset \mathbb{R}^{2 n+1}$.

The smallness of the exceptional set $E(U)$ can be made more precise: all the $t$-slices of $E(U)$ have zero $2 n$-dimensional Lebesgue measure in $\Omega$.

Proof. Our assumption ensures that the function $U$ is locally bounded from above. The first statement follows immediately from (1.1). Since $U$ is locally Lipschitz in $t$, there is no need to regularize in the $t$ variable: it follows from Lemma 1.5 that for all $(t, z) \in \Omega_{T}$,

$$
U^{*}(t, z)=\left(U_{t}\right)^{*}(z),
$$

where the upper semicontinuous regularization in the LHS is in the $(t, z)$ variable, while the upper semicontinuous regularization in the RHS is in the $z$-variable only, $t$ being fixed. A classical theorem of Lelong (see [GZ, Proposition 1.40]) ensures that $E_{t}=\left\{z \in \Omega ; U_{t}(z)<\left(U_{t}\right)^{*}(z)\right\}$ has zero 
Lebesgue measure in $\mathbb{C}^{n}$. Since $E=\left\{(t, z) \in \Omega_{T} ; z \in E_{t}\right\}$ the second statement of the lemma follows from Fubini's theorem.

1.1.2. Semi-continuous regularization. Given a function $u$ on a metric space $(Z, d)$ which is locally bounded from above, we define $\operatorname{usc}_{Z} u$ to be the smallest upper semi-continuous function lying above $u$,

$$
\operatorname{usc}_{Z} u(z):=\limsup _{z^{\prime} \rightarrow z} u\left(z^{\prime}\right)=\inf _{r>0}\left(\sup _{B(z, r)} u\right) .
$$

Fix $I \subset \mathbb{R}$ an interval, $(Y, d)$ a metric space and $\phi: I \times Y \longrightarrow[-\infty, \infty[$ a function. For any $\delta>0$, we denote by $\kappa_{I}(\phi, \delta)$ the smallest constant $\kappa>0$ such that for any $s, t \in I$ with $|s-t| \leq \delta$ and any $y \in Y$,

$$
\phi(t, y) \leq \phi(s, y)+\kappa .
$$

Lemma 1.5. Assume $\phi: I \times Y \longrightarrow\left[-\infty,+\infty\right.$ [ satisfies $\lim _{\delta \rightarrow 0} \kappa_{I}(\phi, \delta)=0$. Then for all $t \in I$ and $y \in Y$,

$$
\left(\operatorname{usc}_{Y} \phi_{t}\right)(y)=\left(\operatorname{usc}_{I \times Y} \phi\right)(t, y) .
$$

In particular if $\phi\left(t_{0}, \cdot\right)$ is upper semi-continuous at a point $y_{0} \in Y$ for some $t_{0} \in I$, then $\phi$ is upper semi-continuous at the point $\left(t_{0}, y_{0}\right) \in I \times Y$.

It follows from Lemma 1.5 that parabolic potentials are upper semicontinuious in $\Omega_{T}$.

We can introduce similarly the lower semi-continuous regularization $\operatorname{lsc}_{Z} u$ of a function $u$ which is locally bounded from below on $Z$. A similar conclusion holds.

Proof. Observe that $\operatorname{usc}_{I \times Y} \phi(t, y) \geq \operatorname{usc}_{Y}\left(\phi_{t}\right)(y)$ for any $(t, y) \in I \times Y$. To prove the reverse inequality, fix $t \in I, y \in Y, A \geq \operatorname{usc}_{Y}\left(\phi_{t}\right)(y)$ and $\varepsilon>0$. Then there exists a neighborhood $V$ of $y$ in $Y$ such that for $z \in V$ we have

$$
\phi(t, z) \leq A+\varepsilon .
$$

Since $\lim _{\delta \rightarrow 0} \kappa_{I}(\phi, \delta)=0$, there exists $\delta>0$ small enough such that $\kappa_{I}(\phi, \delta) \leq$ $\varepsilon$. For $(s, z) \in I \times V$ with $|s-t| \leq \delta$ we have

$$
\phi(s, z) \leq \phi(t, z)+\kappa_{I}(\phi, \delta) \leq A+\varepsilon+\kappa_{I}(\phi, \delta) \leq A+2 \varepsilon .
$$

This proves the inequality $\operatorname{usc}_{I \times Y} \phi(t, y) \leq \operatorname{usc}_{Y}\left(\phi_{t}\right)(y)$.

1.1.3. Approximate submean value inequalities. Parabolic potentials satisfy approximate sub-mean value inequalities:

Lemma 1.6. Let $\Omega \subset \mathbb{C}^{n}$ be a domain and $u \in \mathcal{P}\left(\Omega_{T}\right)$. Fix $\left(t_{0}, x_{0}\right) \in \Omega_{T}$ and $\varepsilon_{0}, r_{0}>0$ so that $\left[t_{0}-\varepsilon_{0}, t_{0}+\varepsilon_{0}\right] \times \bar{B}\left(x_{0}, r_{0}\right) \Subset \Omega_{T}$. Then for any $0<\varepsilon \leq \varepsilon_{0}, 0<r \leq r_{0}$,

$$
u\left(t_{0}, x_{0}\right) \leq \int_{-1}^{1} \int_{\mathbb{B}} u\left(t_{0}+\varepsilon s, x_{0}+r \xi\right) d \lambda_{\mathbb{B}}(\xi) d s / 2+\kappa_{0} \varepsilon,
$$

where $\kappa_{0}>0$ is the uniform Lipschitz constant of $u$ in $\left[t_{0}-\varepsilon_{0}, t_{0}+\varepsilon_{0}\right] \times$ $B\left(x_{0}, r\right)$. 
Proof. Since $u\left(t_{0}, \cdot\right)$ is psh in $\Omega$, the submean-value inequality yields, for all $0<r \leq r_{0}$,

$$
u\left(t_{0}, z_{0}\right) \leq \int_{\mathbb{B}} u\left(t_{0}, z_{0}+r \xi\right) d \lambda_{\mathbb{B}}(\xi) .
$$

The Lipschitz condition ensures that for $0<r \leq r_{0}, 0<\varepsilon \leq \varepsilon_{0}$, and $-1 \leq s \leq 1$,

$$
\int_{\mathbb{B}} u\left(t_{0}, z_{0}+r \xi\right) d \lambda_{\mathbb{B}}(\xi) \leq \int_{\mathbb{B}} u\left(t_{0}+\varepsilon s, z_{0}+r \xi\right) d \lambda_{\mathbb{B}}(\xi)+\kappa_{0} \varepsilon|s| .
$$

Integrating in $s$ we obtain the required inequality.

Parabolic potentials therefore enjoy interesting integrability properties:

Corollary 1.7. We have $\mathcal{P}\left(\Omega_{T}\right) \subset L_{\mathrm{loc}}^{q}\left(\Omega_{T}\right)$ for any $q \geq 1$. Moreover if $u \in \mathcal{P}\left(\Omega_{T}\right)$ then for all $(t, z) \in \Omega_{T}$,

$$
u(t, z)=\lim _{\varepsilon, r \rightarrow 0} \int_{-1}^{1} \int_{\mathbb{B}} u(t+\varepsilon s, z+r \xi) d \lambda_{\mathbb{B}}(\xi) d s / 2 .
$$

In particular if $u, v \in \mathcal{P}\left(\Omega_{T}\right)$ and $u \leq v$ a.e. in $\Omega_{T}$, then $u \leq v$ everywhere.

Proof. Let $u \in \mathcal{P}\left(\Omega_{T}\right)$ and fix $K \Subset \Omega_{T}$ a compact subset. Then there exists a compact interval $J \Subset] 0, T$ [ and a compact subset $D \Subset \Omega$ such that $K \subset J \times D$.

Fix $t_{0} \in J$. Since $u\left(t_{0}, \cdot\right)$ is plurisubharmonic in $\Omega$ we have that $u\left(t_{0}, \cdot\right) \in$ $L^{q}(D)$. Since $u(\cdot, z)$ is uniformly Lipschitz in $J$ we infer $\left|u(t, z)-u\left(t_{0}, z\right)\right| \leq$ $\kappa_{J}\left|t-t_{0}\right|$ for all $t \in J$ and $z \in D$. It thus follows from Fubini's theorem that

$$
\begin{array}{rl}
\int_{J \times D}|u(t, z)|^{q} & d V(z) d t=\int_{J \times D}|u(t, z)|^{q} d V(z) d t \\
& \leq 2^{q-1}\left(\int_{J \times D}\left(\left|u\left(t_{0}, z\right)\right|^{q}+\left|u(t, z)-u\left(t_{0}, z\right)\right|^{q}\right) d V(z) d t\right) \\
& \leq 2^{q-1} \int_{J \times D}\left|u\left(t_{0}, z\right)\right|^{q}+2^{q-1} \kappa_{J}^{q} \operatorname{Vol}(D) \int_{J}\left|t-t_{0}\right|^{q} d t .
\end{array}
$$

This proves that $u \in L^{q}(K)$, hence $u \in L_{\mathrm{loc}}^{q}\left(\Omega_{T}\right)$.

Fix $\left(t_{0}, z_{0}\right) \in \Omega_{T}$ and $\delta>0$. Since $u\left(t_{0}, \cdot\right)$ is psh in $\Omega$ we have

$$
u\left(t_{0}, z_{0}\right)=\lim _{r \rightarrow 0^{+}} \int_{\mathbb{B}} u\left(t_{0}, z_{0}+r \xi\right) d \lambda_{\mathbb{B}}(\xi) .
$$

For small enough $r>0$ we infer

$$
u\left(t_{0}, z_{0}\right) \geq \int_{\mathbb{B}} u\left(t_{0}, z_{0}+r \xi\right) d \lambda_{\mathbb{B}}(\xi)-\delta .
$$

Fix $\varepsilon_{0}>0$ such that $\left.\left[t_{0}-\varepsilon_{0}, t_{0}+\varepsilon_{0}\right] \Subset\right] 0, T\left[\right.$. Let $\kappa_{0}$ be the uniform Lipschitz constant of $u$ in $\left[t_{0}-\varepsilon_{0}, t_{0}+\varepsilon_{0}\right] \times \Omega$. Then for $\left.\varepsilon \in\right] 0, \varepsilon_{0}[$,

$$
u\left(t_{0}, z_{0}\right) \geq \int_{\mathbb{B}} u\left(t_{0}+\varepsilon s, z_{0}+r \xi\right) d \lambda_{\mathbb{B}}(\xi)-\kappa_{0} \varepsilon|s|-\delta .
$$


Integrating in $s \in[-1,1]$ we obtain

$$
u\left(t_{0}, z_{0}\right) \geq \int_{-1}^{1} \int_{\mathbb{B}} u\left(t_{0}+\varepsilon s, z_{0}+r \xi\right) d \lambda_{\mathbb{B}}(\xi) d s / 2-\kappa_{0} \varepsilon-\delta .
$$

Letting $r \rightarrow 0, \varepsilon \rightarrow 0$ and then $\delta \rightarrow 0$ we get one inequality. The reverse inequality was already obtained in Lemma 1.6.

1.2. Behaviour on slices. We now estimate the $L^{1}$-norm on slices in terms of the global $L^{1}$-norm.

Lemma 1.8. Fix $u, v \in \mathcal{P}\left(\Omega_{T}\right)$ and $0<T_{0}<T_{1}<S<T$. Then for all $T_{0} \leq t \leq T_{1}$,

$$
\|u(t, \cdot)-v(t, \cdot)\|_{L^{1}(\Omega)} \leq 2 M \max \left\{\|u-v\|_{L^{1}\left(\Omega_{T_{1}}\right)}^{1 / 2},\|u-v\|_{L^{1}\left(\Omega_{T_{1}}\right)}\right\},
$$

where $M:=\max \left\{\sqrt{\kappa \operatorname{Vol}(\Omega)},\left(S-T_{1}\right)^{-1}\right\}$, and

$$
\kappa:=\sup _{z \in \Omega} \sup _{t, s \in\left[T_{0}, S\right]} \frac{|(u-v)(t, z)-(u-v)(s, z)|}{|t-s|}
$$

is the uniform Lipschitz constant of $u-v$ in $\left[T_{0}, S\right]$.

Here sup* is the essential sup with respect to Lebesgue measure. This lemma quantifies the following facts : for functions in $\mathcal{P}\left(\Omega_{T}\right)$,

- convergence in $L^{1}\left(\Omega_{T}\right)$ implies convergence of their slices in $L^{1}(\Omega)$;

- boundedness in $L^{1}\left(\Omega_{T}\right)$ implies compactness of their slices in $L^{1}(\Omega)$.

Proof. Since $u, v$ are uniformly Lipschitz in $\left[T_{0}, S\right] \times \Omega$, we deduce that for any $T_{0} \leq t \leq S$, and $T_{0} \leq s \leq S$,

$$
|u(t, x)-v(t, x)| \leq \kappa|s-t|+|u(s, x)-v(s, x)|,
$$

where $\kappa>0$ is the uniform Lipschitz constant of $u-v$ on $\left[T_{0}, S\right]$. We infer

$$
\int_{\Omega}|u(t, z)-v(t, z)| d V(z) \leq \kappa|s-t| \operatorname{Vol}(\Omega)+\int_{\Omega}|u(s, z)-v(s, z)| d V(z) .
$$

Thus the function

$$
t \mapsto \theta(t):=\int_{\Omega}|u(t, z)-v(t, z)| d V(z)
$$

is a Lipschitz function in $\left[T_{0}, S\right]$ with Lipschitz constant $\kappa \operatorname{Vol}(\Omega)$. The conclusion follows from the next lemma, an elementary result in one real variable.

We have used the following inequality :

Lemma 1.9. Fix $0<S_{0}<S_{1}<S$ and let $\theta:\left[S_{0}, S\right] \longrightarrow \mathbb{R}$ be such that for all $s, \sigma \in\left[S_{0}, S\right]$ with $s \leq \sigma, \theta(s) \leq \theta(\sigma)+\kappa(\sigma-s)$. Then

$$
\max _{S_{0} \leq s \leq S_{1}} \theta(s) \leq 2 M \max \{\sqrt{\|\theta\|},\|\theta\|\}
$$

where $M:=\max \left\{\sqrt{\kappa},\left(S-S_{1}\right)^{-1}\right\}$ and $\|\theta\|:=\|\theta\|_{L^{1}\left(\left[S_{0}, S\right]\right)}$. 
Proof. Fix $0<\delta \leq S-S_{1}$. Then for $\sigma, s \in\left[S_{0}, S_{1}\right]$ with $s \leq \sigma$,

$$
\theta(s) \leq \theta(\sigma)+\kappa(\sigma-s) .
$$

Fix $S_{0} \leq s \leq S_{1}$. Integrating in $\sigma$ in $[s, s+\delta] \subset\left[S_{0}, S\right]$, we get

$$
\theta(s) \leq \frac{\kappa \delta}{2}+\int_{s}^{s+\delta} \theta(\sigma) \frac{d \sigma}{\delta} \leq \frac{\delta \kappa}{2}+\delta^{-1}\|\theta\|
$$

The minimum of $\tau \longmapsto \kappa \tau / 2+\tau^{-1}\|\theta\|$ is achieved at $\tau_{0}:=\sqrt{2}\|\theta\|^{1 / 2} / \sqrt{\kappa}$. If $2\|\theta\| \leq \kappa\left(S-S_{1}\right)^{2}$ i.e. $\tau_{0} \leq S-S_{1}$, then $\theta(t) \leq 2 \sqrt{\kappa\|\theta\|}$, for $t \in\left[S_{0}, S_{1}\right]$. If $2\|\theta\| \geq \kappa\left(S-S_{1}\right)^{2}$, applying (1.3) with $\delta=S-S_{1}$ yields

$$
\max _{S_{0} \leq t \leq S_{1}} \theta(t) \leq \kappa\left(S-S_{1}\right) / 2+\|\theta\|\left(S-S_{1}\right)^{-1} \leq 2\|\theta\|\left(S-S_{1}\right)^{-1} .
$$

Altogether we obtain

$$
\max _{S_{0} \leq t \leq S_{1}} \theta(t) \leq 2 \max \left\{\sqrt{\kappa\|\theta\|},\|\theta\|\left(S-S_{1}\right)^{-1}\right\} .
$$

1.3. Time derivatives and semi-concavity. In this section we observe that a parabolic potential $\varphi$ has well defined time derivatives $\partial_{t} \varphi$ almost everywhere. Let $\ell$ denote the Lebesgue measure on $\mathbb{R}$, and fix a positive Borel measure $\mu$ on $\Omega$.

Lemma 1.10. Fix $\varphi \in \mathcal{P}\left(\Omega_{T}\right)$. Then there exists a Borel set $E \subset \Omega_{T}$ $\ell \otimes \mu$-negligible such that $\partial_{t} \varphi(t, z)$ exists for all $(t, z) \notin E$.

In particular $\partial_{t} \varphi \in L_{\mathrm{loc}}^{\infty}\left(\Omega_{T}\right)$ and for any continuous function $\gamma \in \mathcal{C}^{0}(\mathbb{R}, \mathbb{R})$, $\gamma\left(\partial_{t} \varphi\right) \ell \otimes \mu$ is a well defined Borel measure in $\Omega_{T}$.

Proof. We set, for $(t, z) \in \Omega_{T}$,

$$
\partial_{t}^{u} \varphi(t, z):=\limsup _{s \rightarrow 0} \frac{\varphi(t+s, z)-\varphi(t, z)}{s}=\limsup _{\mathbb{Q}^{*} \ni s \rightarrow 0} \frac{\varphi(t+s, z)-\varphi(t, z)}{s},
$$

and

$$
\partial_{t}^{l} \varphi(t, z):=\liminf _{s \rightarrow 0} \frac{\varphi(t+s, z)-\varphi(t, z)}{s}=\liminf _{\mathbb{Q}^{*} \ni s \rightarrow 0} \frac{\varphi(t+s, z)-\varphi(t, z)}{s} .
$$

The equalities above follow from the Lipschitz property of $\varphi$. These two functions are measurable in $\left(\Omega_{T}, \ell \otimes \mu\right)$, hence the set

$$
E:=\left\{(t, x) \in \Omega_{T} ; \partial^{l} \varphi(t, z)<\partial^{u} \varphi(t, z)\right\}
$$

is $\ell \otimes \mu$-measurable. For each $z \in \Omega$ fixed, $t \mapsto \varphi(t, z)$ is locally Lipschitz hence differentiable almost everywhere in $] 0, T$. Hence, for all $z \in \Omega$,

$$
E_{z}:=\{t \in] 0, T[;(t, z) \in E\}
$$

has zero $\ell$-measure. Fubini's theorem thus ensures that $\ell \otimes \mu(E)=0$. 
The previous lemma shows that $\partial_{t}^{u} \varphi=\partial_{t}^{l} \varphi, \ell \otimes \mu$-almost everywhere in $\Omega_{T}$. These thus define a function which we denote by $\partial_{t} \varphi \in L_{\mathrm{loc}}^{\infty}\left(\Omega_{T}\right)$.

When $\varphi$ is semi-concave (or semi-convex) in $t$, we can improve the previous result.

Definition 1.11. We say that $\varphi: \Omega_{T} \longrightarrow \mathbb{R}$ is uniformly semi-concave in ] $0, T$ if for any compact $J \Subset] 0, T$, there exists $\kappa=\kappa(J, \varphi)>0$ such that for all $z \in \Omega$, the function $t \longmapsto \varphi(t, z)-\kappa t^{2}$ is concave in $J$.

The definition of uniformly semi-convex functions is analogous. Note that such functions are automatically locally uniformly Lipschitz.

Lemma 1.12. Let $\varphi: \Omega_{T} \longrightarrow \mathbb{R}$ be a continuous function which is uniformly semi-concave in $] 0, T\left[\right.$. Then $(t, z) \mapsto \partial_{t}^{-} \varphi(t, z)$ is upper semi-continuous while $(t, z) \mapsto \partial_{t}^{+} \varphi(t, z)$ is lower semi-continuous in $\Omega_{T}$. In particular, there exists a Borel set $E \subset \Omega_{T}$ which is $\ell \otimes \mu$-negligible, such that $\partial_{t}^{+} \varphi$ and $\partial_{t}^{-} \varphi$ coincide and are continous at each point in $\Omega_{T} \backslash E$.

By replacing $\varphi$ with $-\varphi$ one obtains similar conclusions for uniformly semi-convex functions.

For a bounded parabolic potential $\varphi$ which is locally semi-concave in $t$ the left and right derivatives

$$
\partial_{t}^{+} \varphi(t, z)=\lim _{s \rightarrow 0^{+}} \frac{\varphi(t+s, z)-\varphi(t, z)}{s},
$$

and

$$
\partial_{t}^{-} \varphi(t, z):=\lim _{s \rightarrow 0^{-}} \frac{\varphi(t+s, z)-\varphi(t, z)}{s}
$$

exist for all $t \in] 0, T\left[\right.$, and $\partial_{t} \varphi(t, z)$ exists if $\partial_{t}^{+} \varphi(t, z)=\partial_{t}^{-} \varphi(t, z)$.

Proof. For simplicity we only treat the semi-convex case. It suffices to consider the case when $t \mapsto \varphi(t, z)$ is convex in $] 0, T$, for all $z \in \Omega$. In this case for all $(t, z) \in \Omega_{T}$, the slope function

$$
s \longmapsto p_{s}(t, z):=\frac{\varphi(t+s, z)-\varphi(t, z)}{s},
$$

is monotone increasing on each interval not containing 0 . It is moreover continuous in $(t, z)$. In particular,

$$
\partial_{t}^{+} \varphi(t, z)=\lim _{s \rightarrow 0^{+}} p_{s}(t, z)=\inf _{s>0} p_{s}(t, z),
$$

is upper semi-continuous in $\Omega_{T}$ and

$$
\partial_{t}^{-} \varphi(t, z)=\lim _{s \rightarrow 0^{-}} p_{s}(t, z)=\sup _{s<0} p_{s}(t, z),
$$

is lower semi-continuous in $\Omega_{T}$. This proves the first part of the lemma.

The second part follows from the fact that convex functions are locally Lipschitz in their domain and Lemma 1.10. 
1.4. Compactness properties. We introduce a natural complete metrizable topology on the convex set $\mathcal{P}\left(\Omega_{T}\right)$.

We recall the definition of the Sobolev space $W_{\infty, \text { loc }}^{1,0}\left(\Omega_{T}\right)$ : this is the set of functions $u \in L_{\text {loc }}^{1}\left(\Omega_{T}\right)$ whose partial time derivative (in the sense of distributions) satisfies $\partial_{t} u \in L_{\mathrm{loc}}^{\infty}\left(\Omega_{T}\right)$. It follows from Lemma 1.10 that

$$
\mathcal{P}\left(\Omega_{T}\right) \subset W_{\infty, \mathrm{loc}}^{1,0}\left(\Omega_{T}\right) .
$$

Let $K \subset \Omega$ be a compact subset. The local uniform Lipschitz constant of $\varphi \in \mathcal{P}\left(\Omega_{T}\right)$ on a compact subinterval $\left.J \Subset\right] 0, T$ [is given by

$$
\sup _{t, s \in J, s \neq t} \sup _{z \in K} \frac{|\varphi(s, z)-\varphi(t, z)|}{|s-t|}=\left\|\partial_{t} \varphi\right\|_{L^{\infty}(J \times \Omega)} .
$$

Definition 1.13. We endow $\mathcal{P}\left(\Omega_{T}\right)$ with the semi-norms associated to $W_{\infty, \text { loc }}^{1,0}\left(\Omega_{T}\right)$ : given a compact subset $\left.J \Subset\right] 0, T\left[\right.$ and $u \in W_{\infty, \text { loc }}^{1,0}(\Omega)$, we set

$$
\rho_{J, K}(u):=\left\|\partial_{t} u\right\|_{L^{\infty}(J \times K)}+\int_{J} \int_{K}|u(t, z)| d V(z) d t .
$$

The spaces $L^{q}\left(\Omega_{T}\right)$ are defined with respect to the $(2 n+1)$-dimensional Lebesgue measure in $\Omega_{T}$. For $k, \ell \in \mathbb{N}$ and $q \geq 1$, we denote by $W_{q, l o c}^{k, \ell}\left(\Omega_{T}\right)$ the Sobolev space of Lebesgue measurable functions whose partial derivatives with respect to $t$ up to order $k$ and partial derivatives with respect to $z$ up to order $\ell$ in the sense of distributions are in $L_{\text {loc }}^{q}\left(\Omega_{T}\right)$.

Parabolic potentials enjoy useful compactness properties :

Proposition 1.14. Let $\left(\varphi_{j}\right) \subset \mathcal{P}\left(\Omega_{T}\right)$ be a sequence which

- is locally uniformly bounded from above in $\Omega_{T}$;

- is locally uniformly Lipschitz in ]0,T[;

- does not converge locally uniformly to $-\infty$ in $\Omega_{T}$.

Then $\left(\varphi_{j}\right)$ is bounded in $L_{\mathrm{loc}}^{1}\left(\Omega_{T}\right)$ and there exists a subsequence which converges to some function $\varphi \in \mathcal{P}\left(\Omega_{T}\right)$ in $L_{\text {loc }}^{1}\left(\Omega_{T}\right)$-topology.

If $\left(\varphi_{j}\right)$ converges weakly to $\varphi$ in the sense of distributions in $\Omega_{T}$, then it converges in $L_{\mathrm{loc}}^{q}\left(\Omega_{T}\right)$ for all $q \geq 1$.

The proof is an extension of Hartog's lemma for sequences of plurisubharmonic functions (see e.g. [GZ, Theorem 1.46]).

Proof. We first prove that $\left(\varphi_{j}\right)$ is bounded in $L_{\text {loc }}^{1}\left(\Omega_{T}\right)$. Fix $\left.J \Subset\right] 0, T$ [ and $K \Subset \Omega$. From the assumptions it follows that, for each $t \in J$ fixed, $\varphi_{j}(t, \cdot)$ does not converge locally uniformly in $\Omega$ to $-\infty$. Hence $\varphi_{j}(t, \cdot)$ is bounded in $L_{\text {loc }}^{1}(\Omega, d V)$. The second condition thus ensures that $\left\{\varphi_{j}\right\}$ is uniformly bounded in $L^{1}(J \times K)$.

For each $r \in \mathbb{Q} \cap] 0, T$, there exists a subsequence of $\varphi_{j}(r, \cdot)$ which converges in $L_{\text {loc }}^{1}(\Omega)$ to some plurisubharmonic function $\varphi(r, \cdot)$ in $\Omega$. After a Cantor process we can extract a subsequence from $\left\{\varphi_{j}\right\}$, still denoted by $\left\{\varphi_{j}\right\}$, such that for each $\left.r \in \mathbb{Q} \cap\right] 0, T\left[\right.$, the sequence $\left\{\varphi_{j}(r, \cdot)\right\}$ converges in 
$L_{\text {loc }}^{1}(\Omega)$ to $\varphi(r, \cdot)$. Since the sequence $\left\{\varphi_{j}\right\}$ is locally uniformly Lipschitz in $t$, it follows that the function $(r, z) \mapsto \varphi(r, z)$ is also locally uniformly Lipschitz in $r$. The function $\varphi$ therefore uniquely extends to $] 0, T[\times \Omega$ by

$$
\varphi(t, z):=\lim _{\mathbb{Q} \ni r \rightarrow t} \varphi(r, z) .
$$

Since $\left\{\varphi_{j}\right\}$ is uniformly Lipschitz in $t$ it follows that $\left\{\varphi_{j}(t, \cdot)\right\}$ converges in $L_{\text {loc }}^{1}(\Omega)$ to $\varphi(t, \cdot)$, for all $\left.t \in\right] 0, T[$ and $\varphi$ is locally uniformly Lipschitz in $t \in] 0, T$. The latter then implies that $\varphi \in \mathcal{P}\left(\Omega_{T}\right)$. By Fubini's theorem and dominated convergence it follows that $\left\{\varphi_{j}\right\}$ converges in $L_{\text {loc }}^{1}\left(\Omega_{T}\right)$ to $\varphi$.

We now prove the last statement, assuming that $\varphi \in \mathcal{P}\left(\Omega_{T}\right)$ and that the sequence $\left\{\varphi_{j}\right\}$ converges in the weak sense of distributions to $\varphi$. We claim that for each $t \in] 0, T\left[,\left\{\varphi_{j}(t, \cdot)\right\}\right.$ converges in the sense of distributions in $\Omega$ to $\varphi(t, \cdot)$. Indeed, fix $\left.t_{0} \in\right] 0, T[$ and let $\chi: \Omega \rightarrow \mathbb{R}$ be a smooth test function in $\Omega$. Let $\varepsilon>0$ be a small constant and let $\eta_{\varepsilon}: \mathbb{R} \rightarrow \mathbb{R}^{+}$be a smooth test function which is supported in $\left[t_{0}-\varepsilon, t_{0}+\varepsilon\right]$ and such that $\int_{\mathbb{R}} \eta_{\varepsilon}(t) d t=1$. By assumption,

$$
\lim _{j \rightarrow+\infty} \int_{\Omega_{T}} \varphi_{j}(t, z) \chi(z) \eta_{\varepsilon}(t) d t d V(z)=\int_{\Omega_{T}} \varphi(t, z) \chi(z) \eta_{\varepsilon}(t) d t d V(z) .
$$

Since the sequence $\left\{\varphi_{j}\right\}$ is locally uniformly Lipschitz in $t$, there exists a constant $\kappa_{0}$ depending on $\varepsilon_{0}:=\min \left(t_{0}, T-t_{0}\right) / 2$ such that

$$
\left|\varphi_{j}(t, z)-\varphi_{j}\left(t_{0}, z\right)\right|+\left|\varphi(t, z)-\varphi\left(t_{0}, z\right)\right| \leq \kappa_{0}\left|t-t_{0}\right|,
$$

for all $t \in\left[t_{0}-\varepsilon_{0}, t_{0}+\varepsilon_{0}\right]$ and $z \in \Omega$. We infer

$$
\begin{gathered}
\left|\int_{\Omega_{T}} \varphi_{j}(t, z) \chi(z) \eta_{\varepsilon}(t) d t d V(z)-\int_{\Omega_{T}} \varphi_{j}\left(t_{0}, z\right) \chi(z) \eta_{\varepsilon}(t) d t d V(z)\right| \\
\leq \kappa_{0} \varepsilon \int_{\Omega}|\chi(z)| d V(z) .
\end{gathered}
$$

The same estimate holds for $\varphi$. Combining (1.4) and (1.5) yields

$$
\lim _{j \rightarrow+\infty} \int_{\Omega} \varphi_{j}\left(t_{0}, z\right) \chi(z) d V(z)=\int_{\Omega} \varphi\left(t_{0}, z\right) \chi(z) d V(z)+O(\varepsilon) .
$$

We finally let $\varepsilon \rightarrow 0$ to conclude the proof of the claim.

Classical properties of plurisubharmonic functions now ensure that $\left\{\varphi_{j}\left(t_{0}, \cdot\right)\right\}$ converges in $L_{\text {loc }}^{q}(\Omega)$ to $\varphi\left(t_{0}, \cdot\right)$. Since $\left\{\varphi_{j}\right\}$ is locally uniformly Lipschitz in $t$, we conclude as above that $\left\{\varphi_{j}\right\}$ converges in $L_{\text {loc }}^{q}\left(\Omega_{T}\right)$ to $\varphi$.

Corollary 1.15. The class $\mathcal{P}\left(\Omega_{T}\right)$ is a subset of $L_{\mathrm{loc}}^{q}\left(\Omega_{T}\right)$ for all $q \geq 1$, and the inclusions $\mathcal{P}\left(\Omega_{T}\right) \hookrightarrow L_{\mathrm{loc}}^{q}\left(\Omega_{T}\right)$ are continuous.

The weak topology and the $L_{\text {loc }}^{q}$-topologies are thus all equivalent when restricted to the class $\mathcal{P}\left(\Omega_{T}\right)$. The set $\mathcal{P}\left(\Omega_{T}\right)$ is thus a complete metric space when endowed with any of these topologies.

Lemma 1.16. We have $\mathcal{P}\left(\Omega_{T}\right) \subset W_{\text {loc }}^{1,1}\left(\Omega_{T}\right)$. 
Proof. Fix $u \in \mathcal{P}\left(\Omega_{T}\right)$. The goal is to prove that $u$ has partial derivative (in $t$ and $z)$ in $L_{\text {loc }}^{1}\left(\Omega_{T}\right)$.

We first recall a basic estimate for the gradient of a plurisubharmonic function. Fix $z_{0} \in \Omega$ and $r>0$ such that the polydisc $D\left(z_{0}, 2 r\right)$ is contained in $\Omega$. It follows from [Hörm, Theorem 4.1.8] (see also [GZ, Theorem 1.48] and its proof at page 32-33) that the derivative of any plurisubharmonic function $z \mapsto \varphi(z)$ exists in $L_{\mathrm{loc}}^{p}(\Omega)$ for any $p<2$ and the uniform estimate

$$
\left(\int_{D\left(z_{0}, r\right)}\left|\nabla_{z} \varphi\right|^{p} d V\right)^{1 / p} \leq C(p, r) \int_{D\left(z_{0}, 2 r\right)}|\varphi| d V
$$

holds for a positive constant $C(p, r)$ depending only on $r$ and $p$.

Fix $J \times K$ a compact subset of $\Omega_{T}$. Then by our previous analysis and the compactness of $K$ there exists a constant $C>0$ depending on $K$ and $\operatorname{dist}(K, \partial \Omega)$ and a compact subset $K \Subset L \Subset \Omega$ such that

$$
\left(\int_{K}\left|\nabla_{z} \varphi\right|^{p} d V\right)^{1 / p} \leq C \int_{L}|\varphi| d V
$$

for every $\varphi \in \operatorname{PSH}(\Omega)$.

Now, for each $t \in] 0, T$ [ the derivative of $u$ in $z$ exists and belongs to $L_{\text {loc }}^{p}(\Omega)$ for any $p<2$ (with uniform bound). Since $u$ is locally uniformly Lipschitz in $t$ it follows that $\partial_{t} u(t, z)$ is bounded in $J \times K$ and $u \in L^{1}(J \times L, d t d V)$. Altogether we obtain $u \in W_{\text {loc }}^{1,1}\left(\Omega_{T}\right)$ as desired.

\section{Parabolic Monge-Ampère operators}

2.1. Parabolic Chern-Levine-Nirenberg inequalities. We assume here that $\varphi \in \mathcal{P}\left(\Omega_{T}\right) \cap L_{\mathrm{loc}}^{\infty}\left(\Omega_{T}\right)$. For all $\left.t \in\right] 0, T[$, the function

$$
\Omega \ni z \mapsto \varphi_{t}(z)=\varphi(t, z) \in \mathbb{R}
$$

is psh and locally bounded, hence the Monge-Ampère measures $\left(d d^{c} \varphi_{t}\right)^{n}$ are well defined Borel measures in the sense of Bedford and Taylor [BT76].

We now show that this family depends continuously on $t$ :

Lemma 2.1. Fix $\varphi \in \mathcal{P}\left(\Omega_{T}\right) \cap L_{\mathrm{loc}}^{\infty}\left(\Omega_{T}\right)$ and $\chi$ a continuous test function in $\Omega_{T}$. Then the function

$$
\Gamma_{\chi}: t \longmapsto \int_{\Omega} \chi(t, \cdot)\left(d d^{c} \varphi_{t}\right)^{n}
$$

is continuous in $] 0, T\left[\right.$. Moreover if $\operatorname{Supp}(\chi) \Subset E_{1} \Subset E_{2} \Subset \Omega_{T}$, then

$$
\sup _{0 \leq t<T}\left|\int_{\Omega} \chi(t, \cdot)\left(d d^{c} \varphi_{t}\right)^{n}\right| \leq C \max _{\Omega_{T}}|\chi|\left(\max _{E}|\varphi|\right)^{n},
$$

where $C>0$ is a constant depending only on $\left(E_{1}, E_{2}, \Omega_{T}\right)$.

In particular, $t \longmapsto\left(d d^{c} \varphi_{t}\right)^{n}$ is continuous, as a map from $] 0, T$ [ to the space of positive Radon measures in $\Omega$ endowed with the weak*-topology. 
Proof. We can reduce to the case when the support of $\chi$ is contained in a product of compact subsets $\left.J \times K \subset E^{\circ} \subset\right] 0, T[\times X$.

We first prove (2.1). For any fixed $t \in] 0, T[$,

$$
\left|\int_{\Omega} \chi(t, \cdot)\left(d d^{c} \varphi_{t}\right)^{n}\right| \leq \max _{\Omega_{T}}|\chi| \int_{K}\left(d d^{c} \varphi_{t}\right)^{n} .
$$

Let $L \Subset \Omega$ be a compact subset such that $K \Subset L^{\circ}$ and $J \times L \subset E$. The classical Chern-Levine-Nirenberg inequalities (see [GZ, Theorem 3.9]) ensure that there exists a constant $C=C(K, L)>0$ such that

$$
\int_{\Omega} \chi_{t}\left(d d^{c} \varphi_{t}\right)^{n} \leq C \max _{\Omega}|\chi|\left(\max _{L}\left|\varphi_{t}\right|\right)^{n} \leq C \max _{\Omega_{T}}|\chi|\left(\max _{E}|\varphi|\right)^{n},
$$

where $C>$ depends only on $\left(K, E, \Omega_{T}\right)$. This yields $(2.1)$.

We now prove that $\Gamma_{\chi}$ is continous in $] 0, T[$. Fix compact sets $J \Subset$ ] $0, T\left[, K \Subset \Omega\right.$ such that $\operatorname{Supp}(\chi) \subset J \times K$. The continuity of $\Gamma_{\chi}$ on $] 0, T[\backslash J$ is clear. Fix now $t_{0} \in J$. By the CLN inequality (see [GZ, Theorem 3.9]),

$$
\lim _{t \rightarrow 0} \int_{\Omega}\left|\chi(t, \cdot)-\chi\left(t_{0}, \cdot\right)\right|\left(d d^{c} \varphi_{t}\right)^{n}=0 .
$$

Since $\chi$ is a continuous test function we also have

$$
\lim _{t \rightarrow t_{0}} \int_{\Omega} \chi\left(t_{0}, \cdot\right)\left(d d^{c} \varphi_{t}\right)^{n}=\int_{\Omega} \chi\left(t_{0}, \cdot\right)\left(d d^{c} \varphi_{t_{0}}\right)^{n} .
$$

This proves the continuity of $\Gamma_{\chi}$ at $t_{0}$, finishing the proof.

Definition 2.2. Fix $\varphi \in \mathcal{P}(\Omega) \cap L_{\mathrm{loc}}^{\infty}\left(\Omega_{T}\right)$. The map

$$
\chi \mapsto \int_{\Omega_{T}} \chi d t \wedge\left(d d^{c} \varphi\right)^{n}:=\int_{0}^{T} d t\left(\int_{\Omega} \chi(t, \cdot)\left(d d^{c} \varphi_{t}\right)^{n}\right) .
$$

defines a positive distribution in $\Omega_{T}$ denoted by $d t \wedge\left(d d^{c} \varphi\right)^{n}$, which can be identified with a positive Radon measure in $\Omega_{T}$.

Proposition 2.3. Fix $\varphi \in \mathcal{P}\left(\Omega_{T}\right) \cap L_{\mathrm{loc}}^{\infty}\left(\Omega_{T}\right)$ and let $\left(\varphi^{j}\right)$ be a monotone sequence of functions in $\mathcal{P}\left(\Omega_{T}\right) \cap L_{\text {loc }}^{\infty}\left(\Omega_{T}\right)$ converging to $\varphi$ almost everywhere in $\Omega_{T}$. Then

$$
d t \wedge\left(d d^{c} \varphi^{j}\right)^{n} \rightarrow d t \wedge\left(d d^{c} \varphi\right)^{n}
$$

in the weak sense of measures in $\Omega_{T}$.

Proof. Let $\chi$ be a continuous test function in $\Omega_{T}$. By definition,

$$
\int_{\Omega_{T}} \chi d t \wedge\left(d d^{c} \varphi^{j}\right)^{n}=\int_{0}^{T} d t\left(\int_{\Omega} \chi(t, \cdot)\left(d d^{c} \varphi^{j}(t, \cdot)\right)^{n}\right)=: \int_{0}^{T} F_{j}(t) d t .
$$

It follows from [BT82, Theorem 2.1 and Proposition 5.2] that $F_{j}$ converges to $F$ pointwise in ]0,T [. Lemma 2.1 ensures that $F_{j}$ is uniformly bounded hence the conclusion follows from Lebesgue convergence theorem.

Remark 2.4. The conclusion of Proposition 2.3 also holds if the sequence $\left(\varphi^{j}\right)$ uniformly converges to $\varphi \in \mathcal{P}\left(\Omega_{T}\right)$. 
2.2. Semi-continuity properties. It is difficult to pass to the limit in the parabolic equation, due to the time derivative. We have the following general semi-continuity property :

Lemma 2.5. Let $\left(\nu_{j}\right)$ be positive Borel measures on a topological manifold $Y$ which converge weakly to $\nu$ in the sense of Radon measures on $Y$. Let $v_{j}: Y \longrightarrow \mathbb{R}$ be a locally uniformly bounded sequence of measurable functions which weakly converge to a measurable function $v$ in $L^{2}(Y, \nu)$.

(1) If $\left\|\nu_{j}-\nu\right\| \rightarrow 0$ (total variation) then $\lim _{j} \int_{Y} v_{j} \nu_{j}=\int_{Y} v \nu$ and

$$
\liminf _{j \rightarrow+\infty} e^{v_{j}} \nu_{j} \geq e^{v} \nu
$$

in the weak sense of Radon measures in $Y$.

(2) If $v_{j} \rightarrow v \nu$-a.e. in $Y$ and $\mathcal{M}:=\left\{\nu_{j} ; j \in \mathbb{N}\right\} \cup\{\nu\}$ is uniformly absolutely continuous with respect to a fixed positive Borel measure $\tilde{\nu}$ on $Y$, then for any continuous function $\theta: \mathbb{R} \rightarrow \mathbb{R}$,

$$
\theta\left(v_{j}\right) \nu_{j} \longrightarrow \theta(v) \nu
$$

as $j \rightarrow+\infty$, in the weak sense of Radon measures in $Y$.

Recall that a set $\mathcal{M}$ of positive Borel measures is uniformly absolutely continuous with respect to a positive Borel measure $\tilde{\nu}$ on $Y$ if for any $\delta>0$ there exists $\alpha>0$ such that $\sup _{\sigma \in \mathcal{M}} \sigma(B) \leq \delta$ whenever $B \subset Y$ is a Borel subset with $\tilde{\nu}(B) \leq \alpha$

A typical example is when $\sigma=f_{\sigma} \tilde{\nu}$, where $\sup _{\sigma \in \mathcal{M}} f_{\sigma}$ is $\tilde{\nu}$-integrable. When $\left\|\nu_{j}-\nu\right\| \rightarrow 0$ in the sense of total variation, then the set $\mathcal{M}:=$ $\left\{\nu_{j} ; j \in \mathbb{N}\right\} \cup\{\nu\}$ is uniformly absolutely continuous with respect to $\nu=\tilde{\nu}$.

Proof. We first prove (1). Recall Young's formula which states that

$$
e^{t}=\sup _{s>0}\{s t-s \log s+s\}
$$

for all $t \in \mathbb{R}$. It therefore suffices to prove that for all $s>0$,

$$
\liminf _{j \rightarrow+\infty} e^{v_{j}} \nu_{j} \geq(s v-s \log s+s) \nu
$$

in the weak sense of Radon measures on $Y$. Now for all $s>0$

$$
e^{v_{j}} \nu_{j}=\sup _{s>0}\left\{\left(s v_{j}-s \log s+s\right) \nu_{j}\right\}
$$

so it suffices to prove that $\liminf _{j} v_{j} \nu_{j} \geq v \nu$ in the sense of Radon measures.

Let $\chi$ be a test function on $Y$. Observe that

$$
\int_{Y} \chi v_{j} d \nu_{j}-\int_{Y} \chi v d \nu=\int_{Y} \chi\left(v_{j}-v\right) d \nu+\int_{Y} \chi v_{j} d\left(\nu_{j}-\nu\right) .
$$

The first term converges to zero by weak convergence. Since $\chi v_{j}$ is uniformly bounded by a constant $M$ the absolute value of the second term is less than $M\left\|\nu_{j}-\nu\right\|_{\operatorname{Supp}(\chi)}$, which converges to 0 . 
We now prove $(2)$. Set $f_{j}:=\theta\left(v_{j}\right)$ and $f:=\theta(v)$ and write

$$
\int_{Y} \chi f_{j} d \nu_{j}-\int_{Y} \chi f d \nu=\int_{Y} \chi\left(f_{j}-f\right) d \nu_{j}+\int_{Y} \chi f d\left(\nu_{j}-\nu\right) .
$$

Observe that $g_{j}:=\chi\left(f-f_{j}\right) \rightarrow 0 \nu$ a.e. in $Y$ since $v_{j} \rightarrow v \nu$-a.e. in $Y$. It follows from Egorov's theorem that the sequence $\left(f_{j}\right)$ converges $\tilde{\nu}$-quasi uniformly to $f$. Since the sequence $\left(\nu_{j}\right)$ is uniformly absolutely continuous with respect to $\tilde{\nu}$ it follows that the first term above converges to 0 as $j \rightarrow+\infty$. By Lusin's theorem, the function $f$ is $\tilde{\nu}$-quasi continuous in $Y$, hence the second term also converges to 0 as $j \rightarrow+\infty$, completing the proof of the lemma.

Proposition 2.6. Let $J \subset \mathbb{R}$ be a bounded open interval, $D$ be a bounded open set in $\mathbb{R}^{m}, m \in \mathbb{N}^{*}$, and $0 \leq g \in L^{p}(D)$ with $p>1$. Let $\left(\psi_{j}\right)$ be a sequence of Borel functions in $J \times D$ such that $\left(e^{\psi_{j}} g\right)$ is uniformly bounded in $L^{1}(J \times D, d t d V)$. Assume that there exists $E \subset D$ with zero Lebesgue measure such that for all $z \in D \backslash E, \psi_{j}(\cdot, z)$ converge to a bounded Borel function $\psi(\cdot, z)$ in the sense of distributions on $J$ and

$$
\sup _{j \in \mathbb{N}, z \in D \backslash E}\left|\int_{J} \chi(t, z) \psi_{j}(t, z) d t\right|<+\infty, \text { for all } \chi \in \mathcal{C}_{0}^{\infty}(J \times D) .
$$

Then for any positive smooth test function $\chi \in \mathcal{C}_{0}^{\infty}(J \times D)$,

$$
\int_{J \times D} \chi(t, z) e^{\psi(t, z)} g(z) d t d V \leq \liminf _{j \rightarrow+\infty} \int_{J \times D} \chi(t, z) e^{\psi_{j}(t, z)} g(z) d t d V .
$$

Proof. Fix $C>0$ such that $|\psi| \leq C$ in $X$. Set $\varphi_{j}:=\max \left(\psi_{j},-C\right), j \in \mathbb{N}$. Then $e^{\varphi_{j}}$ is uniformly bounded in $L^{1}(J \times D, g d t d V)$. It follows that $\left(\varphi_{j}\right)$ is bounded in $L^{2}(J \times D, g d t d V)$. Up to extracting and relabelling, it follows from Banach-Saks theorem that the arithmetic mean sequence

$$
\Psi_{N}:=\frac{1}{N} \sum_{j=0}^{N} \varphi_{j}
$$

converges almost everywhere and in $L^{2}(J \times D, g d t d V)$ towards a function $\Psi \in L^{2}(J \times D, g d t d V)$.

Condition (2.2) and Lebesgue's theorem ensure that $\psi_{j} g$ converges in the sense of distributions on $J \times D$ to $\psi g$. This together with the convergence of $\Psi_{N}$ towards $\Psi$ ensure that for any positive smooth test function $\chi$ in $J \times D$,

$$
\begin{aligned}
\int_{J \times D} \chi \Psi g d t d V & =\lim _{N \rightarrow+\infty} \int_{J \times D} \chi \Psi_{N} g d t d V \\
& =\lim _{N \rightarrow+\infty} \frac{1}{N} \sum_{j=1}^{N} \int_{J \times D} \chi \max \left(\psi_{j},-C\right) g d t d V \\
& \geq \lim _{N \rightarrow+\infty} \frac{1}{N} \sum_{j=1}^{N} \int_{J \times D} \chi \psi_{j} g d t d V=\int_{J \times D} \chi \psi g d t d V .
\end{aligned}
$$


This implies that $\Psi g \geq \psi g$ in $L^{1}(J \times D)$, hence $e^{\Psi} g \geq e^{\psi} g$ in $L^{1}(J \times D)$.

It thus follows from Fatou's lemma that

$$
\liminf _{N \rightarrow+\infty} \int_{J \times D} e^{\Psi_{N}} \chi g d t d V \geq \int_{J \times D} e^{\Psi} \chi g d t d V \geq \int_{J \times D} e^{\psi} \chi g d t d V .
$$

It follows now from the convexity of the exponential that

$$
\begin{aligned}
\int_{X} e^{\Psi_{N}} \chi g d t d V & \leq \frac{1}{N} \sum_{j=1}^{N} \int_{X} e^{\varphi_{j}} \chi g d \mu \\
& \leq \frac{1}{N} \sum_{j=1}^{N} \int_{X} e^{\psi_{j}} \chi g d \mu+\int_{X} e^{-C} \chi g d t d V
\end{aligned}
$$

hence letting $N \rightarrow+\infty$ we get

$$
\int e^{\Psi} \chi g d t d V \leq \liminf _{j \rightarrow+\infty} \int_{X} \chi e^{\psi_{j}} g d t d V+e^{-C} \int_{X} \chi g d d t d V .
$$

Letting $C \rightarrow+\infty$ we obtain (2.3).

2.3. Semi-concavity and convergence. In the sequel we need more precise convergence results which require stronger assumptions :

Definition 2.7. A function $\gamma: I \rightarrow \mathbb{R}$ is $\kappa$-concave if $t \mapsto \gamma(t)-\kappa t^{2}$ is concave. It is called locally semi-concave in $I$ if for any subinterval $J \subset I$, there exists $\kappa=\kappa(J, \gamma)>0$ such that $\gamma$ is $\kappa$-concave in $J$.

A family $\mathcal{A}$ of semi-concave functions in some interval $I \subset \mathbb{R}$ is called locally uniformly semi-concave if for any compact subinterval $J \Subset I$, there exists a constant $\kappa=\kappa(J, \mathcal{A})>0$ such that any $\gamma \in \mathcal{A}$ is $\kappa$-concave in $J$.

The following elementary lemma is useful :

Lemma 2.8. Let $\left(\gamma_{j}\right)$ be a sequence of uniformly semi-concave functions in an interval $I \subset \mathbb{R}$ which converges pointwise to a function $\gamma$. Then there exists a countable subset $S \subset I$ such that for all $t \in I \backslash S$, the derivatives $\dot{\gamma}_{j}(t), \dot{\gamma}(t)$ exist and $\lim _{j \rightarrow+\infty} \dot{\gamma}_{j}(t)=\dot{\gamma}(t)$. Moreover if $\dot{\gamma}\left(t_{0}\right)$ exists then

$$
\lim _{j \rightarrow+\infty} \partial_{t}^{-} \gamma_{j}\left(t_{0}\right)=\lim _{j \rightarrow+\infty} \partial_{t}^{+} \gamma_{j}\left(t_{0}\right)=\dot{\gamma}\left(t_{0}\right)
$$

We include a proof for the reader's convenience.

Proof. We can assume that $\gamma_{j}$ is concave in $I$ for all $j$ and $t_{0}=0$. Thus for all $j \in \mathbb{N}$ and $t<0$,

$$
t \partial_{t}^{-} \gamma_{j}(0) \geq \gamma_{j}(t)-\gamma_{j}(0)
$$

Dividing by $t<0$ and taking limits (first $j \rightarrow+\infty$, then $t \rightarrow 0^{-}$), we obtain $\partial_{t}^{-} \gamma(0) \geq \limsup _{j \rightarrow+\infty} \partial_{t}^{-} \gamma_{j}(0)$. Similarly $\liminf _{j \rightarrow+\infty} \partial_{t}^{+} \gamma_{j}(0) \geq$ $\partial_{t}^{+} \gamma(0)$. Since $\partial_{t}^{-} \gamma_{j}(0) \geq \partial_{t}^{+} \gamma_{j}(0)$ we conclude that

$$
\partial_{t}^{-} \gamma(0) \geq \limsup _{j \rightarrow+\infty} \partial_{t}^{-} \gamma_{j}(0) \geq \liminf _{j \rightarrow+\infty} \partial_{t}^{+} \gamma_{j}(0) \geq \partial_{t}^{+} \gamma(0)
$$


If $\dot{\gamma}(0)$ exists, $\partial_{t}^{-} \gamma(0)=\dot{\gamma}(0)=\partial_{t}^{+} \gamma(0)$, hence

$$
\lim _{j \rightarrow+\infty} \partial_{t}^{-} \gamma_{j}(0)=\lim _{j \rightarrow+\infty} \partial_{t}^{+} \gamma_{j}(0)=\dot{\gamma}(0) .
$$

Observe now that the derivatives of a concave function $\partial_{t}^{ \pm} \gamma(t)$ are monotone decreasing, hence continuous outside a countable subset of $I$. Since $\partial_{t}^{+} \gamma(t)=\partial_{t}^{-} \gamma(t)$ almost everywhere by Lemma 1.12, it follows that they are equal outside a countable set in $I$.

We now prove a convergence result that will play a key role in the sequel. We fix $\mu$ a positive Borel measure on $\Omega$ and let $\ell$ denote the Lebesgue measure on $\mathbb{R}$.

Proposition 2.9. Let $\left(f_{j}\right)$ be a sequence of positive functions converging to $f$ in $L^{1}\left(\Omega_{T}, \ell \otimes \mu\right)$. Let $\left(\varphi^{j}\right)$ be a sequence of functions in $\mathcal{P}\left(\Omega_{T}\right)$ which

- converges $\ell \otimes \mu$-almost everywhere in $\Omega_{T}$ to a function $\varphi \in \mathcal{P}\left(\Omega_{T}\right)$;

- is locally uniformly semi-concave in $] 0, T[$.

Then $\lim _{j \rightarrow+\infty} \dot{\varphi}^{j}(t, x)=\dot{\varphi}(t, x)$ for $\ell \otimes \mu$-almost any $(t, x) \in \Omega_{T}$, and

$$
\theta\left(\dot{\varphi}^{j}\right) f_{j} \ell \otimes \mu \rightarrow \theta(\dot{\varphi}) f \ell \otimes \mu,
$$

in the weak sense of Radon measures in $\Omega_{T}$, for all $\theta \in \mathcal{C}^{0}(\mathbb{R}, \mathbb{R})$.

Proof. Fix a compact subinterval $J \Subset] 0, T[$. By definition there exists a constant $\kappa>0$ such that all the functions $t \longmapsto u^{j}(t, x):=\varphi^{j}(t, x)-\kappa t^{2}$ are concave in $J$. By our hypothesis there exists a $\mu$-negligible subset $E_{1} \subset \Omega_{T}$ such that for any $(t, x) \notin E_{1}$, the sequence $u^{j}(t, x)$ converges to $u(t, x):=$ $\varphi(t, x)-\kappa t^{2}$. It follows from Lemma 1.12 and Lemma 2.8 that there exists a $\ell \otimes \mu$-negligeable subset $E_{2} \subset \Omega_{T}$ containing $E_{1}$ such that $\dot{\varphi}^{j}(t, x)$ and $\dot{\varphi}(t, x)$ are well defined for all $j$ and all $(t, x) \notin E_{2}$, with

$$
\lim _{j \rightarrow+\infty} \dot{\varphi}^{j}(t, x)=\dot{\varphi}(t, x) .
$$

Since $f_{j} \rightarrow f$ in $L^{1}\left(\Omega_{T}, \ell \otimes \mu\right)$ we can find $g \in L^{1}\left(\Omega_{T}, \ell \otimes \mu\right)$ such that $0 \leq f_{j} \leq g$ in $\Omega_{T}$. The measures $\left(f_{j} \ell \otimes \mu\right)$ are thus uniformly absolutely continuous with respect to the positive Borel measure $g \ell \times \mu$. The conclusion of the theorem follows therefore from Lebesgue's theorem.

\subsection{Elliptic tools.}

Lemma 2.10. Let $u, v$ be bounded psh functions in $\Omega$ such that

$$
\left(d d^{c} u\right)^{n} \geq e^{f_{1}} \mu \text { and }\left(d d^{c} v\right)^{n} \geq e^{f_{2}} \mu,
$$

where $f_{1}, f_{2}$ are bounded Borel functions in $\Omega$ and $\mu$ is a positive Radon measure with $L^{1}$ density with respect to Lebesgue measure. Then

$$
\left(d d^{c}(\lambda u+(1-\lambda) v)\right)^{n} \geq e^{\lambda f_{1}+(1-\lambda) f_{2}} \mu, \text { for all }, \lambda \in[0,1] .
$$


Proof. Observe first that

$$
\left(d d^{c}(\lambda u+(1-\lambda) v)\right)^{n}=\sum_{k=0}^{n} a_{k}\left(d d^{c} u\right)^{k} \wedge\left(d d^{c} v\right)^{n-k},
$$

where $a_{k} \in(0,1)$, for all $k$ and $\sum_{k=0}^{n} a_{k}=1$. It follows from the mixed Monge-Ampère inequalities [Koł03] (see also [Din09]) that for all $k=0, \cdots n$,

$$
\left(d d^{c} u\right)^{k} \wedge\left(d d^{c} v\right)^{n-k} \geq e^{\left(k f_{1}+(n-k) f_{2}\right) / n} \mu .
$$

Summing up the above inequalities and using the convexity of the exponential yields the desired inequality.

Lemma 2.11. Let $u$ be a psh function in $\Omega$ such that $\lim _{z \rightarrow \zeta} u(z)=\phi(\zeta)$ where $\phi$ is a continuous function on $\partial \Omega$. There exists a decreasing sequence $\left(u_{j}\right)$ of plurisubharmonic functions which are continuous on $\bar{\Omega}$ and such that $u_{j}=\phi$ on $\partial \Omega$ and $u_{j} \searrow u$ in $\Omega$.

This result is classical but we include a proof for the reader's convenience.

Proof. It follows from the strictly pseudoconvex assumption on $\Omega$ that there exists a harmonic function $\Phi$ in $\Omega$ with boundary value $\phi$. We first take a sequence of continuous functions $\left\{f_{j}\right\} \subset \mathcal{C}(\bar{\Omega})$ which decreases pointwise to $u$ in $\bar{\Omega}$. By considering $\min \left(f_{j}, \Phi\right)$ we can assume that $f_{j}=\phi$ on $\partial \Omega$. For each $j$, consider the psh envelope

$$
u_{j}:=P\left(f_{j}\right):=\sup \left\{v \in \operatorname{PSH}(\Omega) ; v^{*} \leq f_{j} \text { in } \bar{\Omega}\right\} .
$$

Then $u \leq u_{j} \leq f_{j}$ and $u_{j} \downarrow u$. Hence $\left(u_{j}\right)_{*}=\left(u_{j}\right)^{*}=\phi$ on $\partial \Omega$. It thus follows from [Wal69, Lemma 1] (see also [Bło05, Proposition 3.2]) that $u_{j}$ is continuous in $\bar{\Omega}$.

\section{BoundARY BEHAVIOR OF PARABOLIC ENVELOPES}

Our aim is to solve the Cauchy-Dirichlet problem for (CMAF) with compatible boundary data $h$ using the Perron method of upper envelopes. In this section we prove that, under natural assumptions, the parabolic Perron envelope has the right boundary values. We assume $T<+\infty$.

3.1. Parabolic pluripotential subsolutions. Recall that for $u \in \mathcal{P}\left(\Omega_{T}\right)$ the time derivative $\partial_{t} u$ exists a.e. in $\Omega_{T}$ and satisfies the local uniform bound $\left|\partial_{t} u\right| \leq \kappa_{J}(u)$ in $J \times \Omega$, for each $\left.J \Subset\right] 0, T[$ (see Lemma 1.10).

Definition 3.1. Fix $u \in \mathcal{P}\left(\Omega_{T}\right) \cap L^{\infty}\left(\Omega_{T}\right)$. The function $u$ is called a pluripotential subsolution to (CMAF) if it satisfies the inequality

$$
d t \wedge\left(d d^{c} u\right)^{n} \geq e^{\dot{u}+F(t, x, u)} g d t \wedge d V
$$

in the sense of measures in $\Omega_{T}$. It is called a pluripotential supersolution to (CMAF) if the reverse inequality holds in the sense of measures in $\Omega_{T}$. 
If moreover $u^{*} \leq h$ in $\partial_{0} \Omega_{T}$, we say that $u$ is a pluripotential subsolution to the Cauchy-Dirichlet problem for the parabolic complex Monge-Ampère equation (CMAF) with boundary data $h$. Here

$$
u^{*}(\tau, \zeta):=\limsup _{\Omega_{T} \ni(t, z) \rightarrow(\tau, \zeta)} u(t, z), \quad(\tau, \zeta) \in \partial_{0} \Omega_{T} .
$$

Proposition 3.2. Fix $u \in \mathcal{P}\left(\Omega_{T}\right) \cap L_{\mathrm{loc}}^{\infty}\left(\Omega_{T}\right)$.

1) $u$ is a pluripotential subsolution to (CMAF) if and only if for a.e. $t$,

$$
\left(d d^{c} u_{t}\right)^{n} \geq e^{\partial_{t} u(t, \cdot)+F\left(t, \cdot, u_{t}\right)} g d V
$$

in the sense of measures in $\Omega$.

2) If $u$ is moreover locally semi-concave in $t$, it is a pluripotential subsolution to (CMAF) if and only if for all $t$,

$$
\left(d d^{c} u_{t}\right)^{n} \geq e^{\partial_{t}^{+} u(t, \cdot)+F\left(t, \cdot, u_{t}\right)} g d V
$$

in the sense of measures in $\Omega$.

Proof. Recall that $\partial_{t} u$ makes sense almost everywhere and, in case $u$ is semi-concave, coincides with $\partial_{t}^{+} u$ which is well defined at every point.

Assume first that (3.1) holds for a.e. $t$. Let $\chi \in \mathcal{C}_{0}^{\infty}\left(\Omega_{T}\right)$ be a nonnegative test function. Multiplying (3.1) by $\chi$ and integrating in $x$ we obtain

$$
\int_{\Omega} \chi(t, x)\left(d d^{c} u_{t}\right)^{n} \geq \int_{\Omega} \chi(t, x) e^{\partial_{t} u+F\left(t, x, u_{t}\right)} g(x) d V(x) .
$$

Integrating with respect to $t$, we infer

$$
\int_{\Omega_{T}} \chi(t, x)\left(d d^{c} u_{t}\right)^{n} \wedge d t \geq \int_{\Omega_{T}} \chi(t, x) e^{\partial_{t} u+F\left(t, x, u_{t}\right)} g(x) d V(x) \wedge d t,
$$

i.e. $u$ is a pluripotential subsolution to (CMAF).

Assume now that $u$ is a pluripotential subsolution to (CMAF). We consider product of nonnegative test functions

$$
\chi(t, x)=\alpha(t) \theta_{j}(x)
$$

where $\left(\theta_{j}\right)$ is a sequence of test functions in $\Omega$ which generates a dense subspace of the space of test functions (for the $\mathcal{C}^{0}$-topology). It follows from Fubini theorem that

$$
\int_{0}^{T}\left\{\int_{\Omega} \theta_{j}(x)\left(d d^{c} u_{t}\right)^{n}\right\} \alpha(t) d t \geq\left\{\int_{\Omega} \theta_{j}(x) e^{\partial_{t} u+F\left(t, x, u_{t}\right)} g(x) d V(x)\right\} \alpha(t) d t .
$$

We infer that for all $t \in B_{j} \subset[0, T[$,

$$
\int_{\Omega} \theta_{j}(x)\left(d d^{c} u_{t}\right)^{n} \geq \int_{\Omega} \theta_{j}(x) e^{\partial_{t} u+F\left(t, x, u_{t}\right)} g(x) d V(x)
$$

where $B_{j}$ has full measure in $\left[0, T\left[\right.\right.$. The set $B=\cap_{j} B_{j} \subset[0, T[$ has full measure and the previous inequality holds for all $t \in B$ and for all $j \in \mathbb{N}$. 
Approximating an arbitrary nonnegative test function $\theta \in \mathcal{C}^{0}(\Omega)$ by convex combinations of the $\theta_{j}$ 's, we infer that for almost every $t$,

$$
\left(d d^{c} u_{t}\right)^{n} \geq e^{\partial_{t} u(t, \cdot)+F\left(t, \cdot, u_{t}\right)} g d V .
$$

When $u$ is moreover locally semi-concave in $t$ the function $\partial_{t}^{+} u$ is lower semi-continuous (see Lemma 1.12), hence

$$
t \mapsto \int_{\Omega} \chi(x) e^{\partial_{t}^{+} u(t, x)+F\left(t, x, u_{t}(x)\right)} g(x) d V(x)
$$

is lower semi-continuous by Fatou's lemma. Since $t \mapsto \int_{\Omega} \chi\left(d d^{c} u_{t}\right)^{n}$ is continuous (by Lemma 2.1), we infer that (3.1) holds for almost every $t$ if and only if it holds for every $t$.

Remark 3.3. A similar result holds in this case, using the partial derivative $\partial_{t}^{-} u$ which is upper semi-continuous when $u$ is locally semi-concave (by Lemma 1.12 again). As a consequence, if $u \in \mathcal{P}\left(\Omega_{T}\right) \cap L_{\text {loc }}^{\infty}\left(\Omega_{T}\right)$ solves (CMAF) and $u$ is locally uniformly semi-concave in $t \in] 0, T$ [ then for almost all $t \in] 0, T[$,

$$
\left(d d^{c} u_{t}\right)^{n}=e^{\partial_{t} u_{t}+F\left(t, \cdot, u_{t}\right)} g d V .
$$

Lemma 3.4. For any $u, v \in \mathcal{P}\left(\Omega_{T}\right) \cap L_{\text {loc }}^{\infty}\left(\Omega_{T}\right)$, we have

$$
\mathbf{1}_{\{u \geq v\}} \partial_{t} \max (u, v)=\mathbf{1}_{\{u \geq v\}} \partial_{t} u \text { and } \mathbf{1}_{\{u>v\}} \partial_{t} \max (u, v)=\mathbf{1}_{\{u>v\}} \partial_{t} u
$$

almost everywhere in $\Omega_{T}$ and

$$
\left(d d^{c} \max (u, v)\right)^{n} \wedge d t \geq \mathbf{1}_{\{u>v\}}\left(d d^{c} u\right)^{n} \wedge d t+\mathbf{1}_{\{u \leq v\}}\left(d d^{c} v\right)^{n} \wedge d t .
$$

In particular the maximum of two subsolutions is again a subsolution.

Proof. It follows from Lemma 1.16 that $\mathcal{P}\left(\Omega_{T}\right) \subset W_{\text {loc }}^{1,1}\left(\Omega_{T}\right)$. The first identity is then a classical result in the theory of Sobolev spaces (see e.g. [GT01, Lemma 7.6 page 152]). The second inequality is a consequence of the elliptic maximum principle for psh functions (see e.g. [GZ, Corollary 3.28]).

It is therefore natural to consider the Perron envelope of subsolutions :

Definition 3.5. We let $\mathcal{S}_{h, g, F}\left(\Omega_{T}\right)$ denote the set of $u \in \mathcal{P}\left(\Omega_{T}\right)$ such that

(1) $u$ is a pluripotential subsolution to $(\mathrm{CMAF})$ in $\Omega_{T}$;

(2) $u^{*} \leq h$ on $\partial_{0} \Omega_{T}$, i.e. for all $(s, \zeta) \in \partial_{0} \Omega_{T}$,

$$
\limsup _{\Omega_{T} \ni(t, z) \rightarrow(s, \zeta)} u(t, z) \leq h(s, \zeta) .
$$

We let

$$
U=U_{h, g, F, \Omega_{T}}:=\sup \left\{u ; u \in \mathcal{S}_{h, g, F}\left(\Omega_{T}\right)\right\}
$$

denote the upper envelope of all subsolutions.

Lemma 3.6. The set $\mathcal{S}_{h, g, F}\left(\Omega_{T}\right)$ is not empty, uniformly bounded in $\Omega_{T}$, stable under finite maxima. The envelope $U:=U_{h, g, F, \Omega_{T}}$ and its upper semi-continuous regularization $U^{*}$ satisfy for all $(t, z) \in \Omega_{T}$,

$$
B \rho(z)-M_{h} \leq U(t, z) \leq U^{*}(t, z) \leq M_{h},
$$


where $B=e^{M_{F} / n}$. In particular

$$
\|U\|_{L^{\infty}\left(\Omega_{T}\right)} \leq M_{U}:=M_{h}+c_{n} e^{M_{F}}\|g\|_{L^{p}(\Omega)}^{1 / n} .
$$

Recall that

$$
M_{h}:=\sup _{\partial_{0} \Omega_{T}}|h|, M_{F}:=\sup _{\Omega_{T}} F\left(\cdot, \cdot, M_{h}\right) .
$$

Proof. Fix $B=e^{M_{F} / n}$. Since $g d V=\left(d d^{c} \rho\right)^{n}$ we obtain

$$
e^{M_{F}} g d V \leq B^{n}\left(d d^{c} \rho\right)^{n} .
$$

Set, for $(t, z) \in \Omega_{T}$,

$$
\underline{u}(t, z):=B \rho(z)-M_{h} .
$$

Then $\underline{u} \in \mathcal{S}_{h, g, F}\left(\Omega_{T}\right)$, hence $\underline{u} \leq U_{h, g, F, \Omega_{T}}$.

Fix $u \in \mathcal{S}_{h, g, F}\left(\Omega_{T}\right)$ and fix $\left.t \in\right] 0, T\left[\right.$. Then $\lim \sup _{z \rightarrow \zeta} u(t, z) \leq h(t, \zeta)$, for every $\zeta \in \partial \Omega$. It thus follows from the classical maximum principle for plurisubharmonic functions that $u(t, z) \leq M_{h}$ for every $z \in \Omega$. Thus $U(t, \cdot) \leq M_{h}$ for any $\left.t \in\right] 0, T[$.

Therefore, the upper envelope $U$ is well defined and satisfies the uniform estimates $\underline{u} \leq U \leq M_{h}$, in $\Omega_{T}$, hence

$$
U(t, z):=\sup \left\{u(t, z) ; u \in \mathcal{S}_{h, g, F}\left(\Omega_{T}\right), \underline{u} \leq u \leq M_{h}\right\} .
$$

The stability under finite maxima follows from Lemma 3.4.

3.2. Construction of sub-barriers. The family $t \longmapsto h(t, z)(z \in \partial \Omega)$ is uniformly Lipschitz in $] 0, T$ [ if there exists a constant $\kappa(h)>0$ such that

$$
|h(t, z)-h(s, z)| \leq \kappa(h)|t-s|, \forall(t, s) \in\left[0, T\left[^{2}, \forall z \in \partial \Omega .\right.\right.
$$

The parabolic boundary of $\Omega_{T}$ consists in two different types of points. We provide barriers for each type.

3.2.1. Sub-barriers at boundary points of Dirichlet type. We first construct subbarriers at Dirichlet boundary points in $[0, T[\times \partial \Omega$.

Lemma 3.7. Assume $h$ satisfies (3.3). Then there exists $u \in \mathcal{S}_{h, g, F}\left(\Omega_{T}\right)$ such that $u(\cdot, z)(z \in \Omega)$ is uniformly Lipschitz in $[0, T[$ and satisfies : for any $(s, \zeta) \in[0, T[\times \partial \Omega$,

$$
\lim _{(t, z) \rightarrow(s, \zeta)} u(t, z)=h(s, \zeta) .
$$

If $h_{0}$ is continuous on $\bar{\Omega}$ then $u$ can be chosen to be continuous in $[0, T[\times \bar{\Omega}$.

Proof. Fix $t \in\left[0, T\left[\right.\right.$ and set $h_{t}:=h(t, \cdot) \in \mathcal{C}(\partial \Omega)$. Let $\phi_{t}$ be the unique continuous plurisubharmonic function in $\Omega$ such that

$$
\left\{\begin{array}{l}
\left(d d^{c} \phi_{t}\right)^{n}=0 \text { in } \Omega, \\
\lim _{z \rightarrow \zeta} \phi_{t}(z)=h_{t}(\zeta)-h_{0}(\zeta), \forall \zeta \in \partial \Omega .
\end{array}\right.
$$

The existence and continuity of $\phi_{t}$ on $\bar{\Omega}$ follows from classical results in pluripotential theory (see [BT76, BT82], [GZ, Theorem 5.12]). Moreover, 
$\phi_{t}$ can be characterized as the supremum of all subsolutions to (3.4). Since $t \longmapsto h(t, z)(z \in \partial \Omega)$ is uniformly Lipschitz in [0,T[, the tautological maximum principle reveals that the family of functions $t \longmapsto \phi(t, z):=\phi_{t}(z)$ $(z \in \Omega)$ is uniformly Lipschitz in $[0, T$ [ with a Lipschitz constant $\kappa(\phi) \leq$ $\kappa(h)$. By Lemma 1.5, $(t, z) \longmapsto \phi_{t}(z)$ is continuous in $[0, T[\times \bar{\Omega}$. Consider now, for $(t, z) \in \Omega_{T}$,

$$
u(t, z):=\phi_{t}(z)+h_{0}(z)+A \rho(z),
$$

where $A>0$ is a large constant to be chosen later, and $\rho$ is defined in (0.7). Observe that $u \in \mathcal{P}\left(\Omega_{T}\right)$ and $u^{*} \leq h$ in $\partial_{0} \Omega_{T}$. It is clear that $t \longmapsto u(t, z)$ $(z \in \Omega)$ is uniformly Lipschitz in $[0, T[$ with $\kappa(u) \leq \kappa(h)$. Moreover

$$
d t \wedge\left(d d^{c} u\right)^{n} \geq A^{n} d t \wedge\left(d d^{c} \rho\right)^{n} \geq A^{n} d t \wedge g d V
$$

in the weak sense of measures in $\Omega_{T}$. We choose $A>0$ so that $n \log A \geq$ $\kappa(h)+M_{F}$. It is then clear that $u \in \mathcal{S}_{h, g, F}\left(\Omega_{T}\right)$. By definition, $u$ is continuous in $\left[0, T\left[\times \Omega\right.\right.$ provided that $h_{0}$ is continuous on $\bar{\Omega}$.

3.2.2. Sub-barriers at boundary points of Cauchy type. We now construct sub-barriers at boundary points in $\{0\} \times \Omega$.

Lemma 3.8. Assume $h$ satisfies (3.3). Then there exists $v \in \mathcal{S}_{h, g, F}\left(\Omega_{T}\right)$ such that for all $\zeta \in \bar{\Omega}$,

$$
\limsup _{\Omega_{T} \ni(t, z) \rightarrow(0, \zeta)} v(t, z)=h_{0}(\zeta) \text {, and } \lim _{t \rightarrow 0^{+}} v(t, \zeta)=h_{0}(\zeta) .
$$

If $h_{0}$ is continuous on $\bar{\Omega}$ then $v$ can be chosen to be continuous on $[0, T[\times \bar{\Omega}$.

Proof. By assumption on $h$ we have, for all $(t, z) \in[0, T[\times \partial \Omega$,

$$
h(0, z) \leq h(t, z)+\kappa t .
$$

Set, for $(t, z) \in \Omega_{T}$,

$$
\left.v(t, z):=h_{0}(z)+t(\rho(z)-C)+n[(t / T) \log (t / T)-t / T)\right],
$$

where $C:=\kappa_{h}+M_{F}-\min (n \log T, 0)$. Then $v \in \mathcal{S}_{h, g, F}\left(\Omega_{T}\right)$ and $v$ is continuous on $\left[0, T\left[\times \bar{\Omega}\right.\right.$ if $h_{0}$ is continuous on $\bar{\Omega}$.

\subsection{Super-barriers.}

3.3.1. Super-barriers at boundary points of Dirichlet type. For each $t \in$ $\left[0, T\right.$, we let $H_{t}$ be the unique harmonic function in $\Omega$ with boundary value $h_{t}$ on $\partial \Omega$ and set $H(t, z):=H_{t}(z)$ (the existence of $H_{t}$ is a classical fact; see e.g. [GT01, Theorem 2.14]). Recall that $H_{t}$ can be defined as the upper envelope of all subharmonic functions in $\Omega$ with boundary values $\leq h_{t}$. Observe that $h_{0} \leq H(0, \cdot)$ in $\Omega$, with equality at the boundary.

Lemma 3.9. For all $(t, z) \in\left[0, T\left[\times \Omega\right.\right.$ we have $U^{*}(t, z) \leq H(t, z)$. In particular, for all $(s, \zeta) \in[0, T[\times \partial \Omega$,

$$
\limsup _{(t, z) \rightarrow(s, \zeta)} U^{*}(t, z) \leq h(s, \zeta) .
$$


Proof. It follows directly from the maximum principle for subharmonic functions that $U_{t} \leq H_{t}$, for all $t \in[0, T[$. Fix $S \in] 0, T$. Since the family $\{h(\cdot, z) ; z \in \partial \Omega\}$ is equicontinuous in $[0, S]$, it follows by definition that the family $\{H(\cdot, z) ; z \in \bar{\Omega}\}$ is equicontinuous in $[0, S]$, hence the function $H$ is continuous in $\left[0, T\left[\times \bar{\Omega}\right.\right.$, by Lemma 1.5. Then $U^{*}(t, z) \leq H(t, z)$ for any $(t, z) \in \Omega_{T}$. From the continuity of $H$, it follows that $U^{*} \leq H$ in $[0, T[\times \bar{\Omega}$.

3.3.2. Boundary behaviour at Cauchy boundary points. So far we have constructed enough barriers to ensure that the envelope of subsolutions either matches the boundary data (at Dirichlet points), or stays below it. The following average argument will allow us to conclude that it also coincides with the boundary data at Cauchy points :

Lemma 3.10. Let $\varphi \in \mathcal{P}\left(\Omega_{T}\right) \cap L^{\infty}\left(\Omega_{T}\right)$ be a subsolution to (CMAF) such that $\int_{D}\left(d d^{c} \varphi_{t}\right)^{n} \leq C$, for every $t \in[0, T[$, for some $C>0$, where $D$ is an open set in $\Omega$. Then, for each positive continuous test function $\chi$ in $D$, there exists $A>0$ such that

$$
t \mapsto \int_{D} \chi \varphi_{t} g d V-A t
$$

is decreasing in $] 0, T[$.

Proof. Since $\varphi$ is a subsolution to (CMAF) we obtain for a.e. $t \in] 0, T[$,

$$
\int_{D} \chi e^{\dot{\varphi}_{t}+m_{F}} g d V \leq \int_{D} \chi e^{\dot{\varphi}_{t}+F} g d V \leq \int_{D} \chi\left(d d^{c} \varphi_{t}\right)^{n} \leq C,
$$

where $m_{F}:=\inf _{\left[0, T\left[\times \bar{\Omega} \times\left[-M_{U}, M_{U}\right]\right.\right.} F$. It follows from Jensen's inequality that

$$
\int_{D} \chi \dot{\varphi}_{t} g d V \leq C_{2}
$$

for a.e. $t \in] 0, T\left[\right.$, where $C_{2}>0$ is a uniform constant. We then infer that the function $t \mapsto \int_{D} \chi \varphi_{t} g d V-C_{2} t$ is decreasing in $] 0, T[$.

Corollary 3.11. Assume $\left\{u^{j}\right\} \subset \mathcal{S}_{h, g, F}\left(\Omega_{T}\right)$ is a bounded sequence which is locally uniformly Lipschitz in $] 0, T$ [ (with Lipschitz constant independing of $j)$. If $\left\{u^{j}\right\}$ converges in $L_{\text {loc }}^{1}\left(\Omega_{T}\right)$ to $u \in \mathcal{P}\left(\Omega_{T}\right)$ then

$$
\limsup _{(t, z) \rightarrow(s, \zeta)} u(t, z) \leq h(s, \zeta), \forall(s, \zeta) \in \partial_{0} \Omega_{T} .
$$

Proof. For $(s, \zeta) \in] 0, T[\times \partial \Omega$ the desired inequality holds thanks to Lemma 3.9. Fix $D \Subset \Omega$ and let $\chi$ be a positive continuous test function in $\Omega$. It follows from the Chern-Levine-Nirenberg inequality [GZ, Theorem 3.9] that $\int_{D}\left(d d^{c} u_{t}^{j}\right)^{n}$ is uniformly bounded. Lemma 3.10 therefore provides us with a uniform constant $A>0$ such that

$$
\left.\int_{D} \chi u_{t}^{j} g d V \leq \int_{D} \chi h_{0} g d V+A t, \forall t \in\right] 0, T[, \forall j .
$$


Letting $j \rightarrow+\infty$, Lemma 1.8 ensures that

$$
\left.\int_{D} \chi u_{t} g d V \leq \int_{D} \chi h_{0} g d V+A t, \forall t \in\right] 0, T[.
$$

If $v$ is a cluster point of $u_{t}$ as $t \rightarrow 0$ then the above estimate yields $v \leq h_{0}$ on $D$. Since $D$ was chosen arbitrarily, $v \leq h_{0}$ on $\Omega$. The conclusion thus follows from Lemma 1.3.

\subsection{Boundary behavior of the Perron envelope.}

Theorem 3.12. Assume $h$ satisfies (3.3). Then the upper semi-continuous regularization of the envelope $U=U_{h, g, F, \Omega_{T}}$ satisfies

(i) for any $(s, \zeta) \in\left[0, T\left[\times \partial \Omega, \lim _{\Omega_{T} \ni(t, z) \rightarrow(s, \zeta)} U^{*}(t, z)=h(s, \zeta)\right.\right.$.

(ii) for any $z_{0} \in \Omega$,

$$
\lim _{t \rightarrow 0^{+}} U^{*}\left(t, z_{0}\right)=h\left(0, z_{0}\right), \text { and } \limsup _{\Omega_{T} \ni(t, z) \rightarrow\left(0, z_{0}\right)} U^{*}(t, z)=h\left(0, z_{0}\right) .
$$

Here $U^{*}$ denotes the u.s.c. regularization of $U$ in the variable $(t, z)$ in $\Omega_{T}$.

Proof. Fix $(s, \zeta) \in[0, T[\times \partial \Omega$. Lemma 3.7 and Lemma 3.9 yield $(i)$.

In view of Lemma 3.8 it remains to prove that for all $z_{0} \in \Omega$,

$$
\limsup _{\Omega_{T} \ni(t, z) \rightarrow\left(0, z_{0}\right)} U^{*}(t, z) \leq h_{0}\left(z_{0}\right) .
$$

The envelope $U$ is locally uniformly Lipschitz in $] 0, T[$, as follows from Theorem 4.2. We can thus apply Lemma 1.4 to conclude that $U^{*}(t, \cdot)=U_{t}^{*}$ in $\Omega$ for any $t \in] 0, T$, where $U_{t}^{*}=\left(U_{t}\right)^{*}$ is the u.s.c. regularization of the function $U_{t}$ ( $t$ fixed) in $\Omega$. Using Lemma 1.3 it is then enough to show that

$$
\limsup _{t \rightarrow 0} U_{t}^{*}\left(z_{0}\right) \leq h_{0}\left(z_{0}\right), \forall z_{0} \in \Omega .
$$

Observe that $U$ can be seen as the upper envelope of all $\varphi \in \mathcal{S}_{h, g, F}\left(\Omega_{T}\right)$ such that $\sup _{\Omega_{T}}|\varphi| \leq M_{U}$, where $M_{U}$ is given in Lemma 3.6.

Fix $\chi$ a continuous positive test function in $\Omega$. We claim that there exists a constant $C>0$ such that for all $t \in] 0, T[$,

$$
\int_{\Omega_{1}} \chi U_{t}^{*} g d V \leq \int_{\Omega_{1}} \chi h_{0} g d V+C t .
$$

Indeed, fix $\left.t_{0} \in\right] 0, T$. Since the set of subsolutions is stable under maximum, by Choquet's lemma, $U_{t_{0}}^{*}=\left(\lim _{j \rightarrow+\infty} \varphi_{t_{0}}^{j}\right)^{*}$ in $\Omega$, where $\left\{\varphi^{j}\right\}$ is an increasing sequence in $\mathcal{S}_{h, g, F}\left(\Omega_{T}\right)$ with $\left|\varphi^{j}\right| \leq M_{U}$. The sequence $\left\{\varphi^{j}\right\}$ depends on $t_{0}$ but, as will be shown later, the constant $C$ does not depend on $t_{0}$. Now fix $j \in \mathbb{N}, \Omega_{1} \Subset \Omega_{2} \Subset \Omega$ compact subsets of $\Omega$. It follows from the Chern-Levine-Nirenberg inequality [GZ, Theorem 3.9] that

$$
\left.\int_{\Omega_{1}} \chi\left(d d^{c} \varphi_{t}^{j}\right)^{n} \leq C_{1}, \text { for all } t \in\right] 0, T[
$$


where $C_{1}$ depends only on $\Omega_{1}, \Omega_{2}, \chi$ and $M_{U}$. It thus follows from Lemma 3.10 that

$$
\left.\int_{\Omega_{1}} \chi \varphi_{t}^{j} g d V \leq \int_{\Omega_{1}} \chi h_{0} g d V+C_{2} t, \text { for all } t \in\right] 0, T[,
$$

for a uniform constant $C_{2}>0$. A classical theorem of Lelong (see [GZ, Proposition1.40]) ensures that

$$
\left\{z \in \Omega ; \lim _{j \rightarrow+\infty} \varphi_{t_{0}}^{j}(z)<\left(U_{t_{0}}\right)^{*}(z)\right\}
$$

has volume zero in $\Omega$. Therefore taking the limit as $j \rightarrow+\infty$ in the previous inequality for $t=t_{0}$, we deduce that

$$
\int_{\Omega_{1}} \chi U_{t_{0}}^{*} g d V \leq \int_{\Omega_{1}} \chi h_{0} g d V+C_{2} t_{0}
$$

Since $C_{2}$ does not depend on $t_{0}$, the claim is proved.

Let $w_{0} \in \operatorname{PSH}(\Omega)$ be any cluster point of $U_{t}^{*}$ as $t \rightarrow 0^{+}$. We can assume that $U_{t}^{*}$ converge to $w_{0}$ in $L^{q}(\Omega)$ for any $q>1$. Then $U_{t}^{*} g$ converge to $w_{0} g$ in $L^{1}(\Omega)$. Thus, by (3.5), $\int_{\Omega_{1}} \chi w_{0} g d V \leq \int_{\Omega_{1}} \chi h_{0} g d V$. Since $\chi \geq 0$ was chosen arbitrarily, we infer that $w_{0} \leq h_{0}$ almost everywhere in $\Omega_{1}$ with respect to $g d V$. The assumption on $g$ finally yields $w_{0} \leq h_{0}$ on $\Omega_{1}$. By letting $\Omega_{1} \rightarrow \Omega$ we can then conclude that $\limsup _{t \rightarrow 0} U_{t}^{*} \leq h_{0}$ in $\Omega$.

Lemma 3.13. If $h_{0}$ is continuous on $\bar{\Omega}$ then $U^{*}(t, \cdot)$ uniformly converges to $h_{0}$ as $t \rightarrow 0^{+}$.

Note that in Lemma 3.13 we merely assume that $h$ is locally uniformly Lipschitz in $t \in] 0, T[$.

Proof. We first assume that $h$ satisfies (3.3). It follows from Lemma 3.8 that there exists a continuous subsolution $u \in \mathcal{S}_{h, g, F}\left(\Omega_{T}\right)$ :

$$
u(t, z):=h_{0}(z)+t(\rho(z)-C)+\eta(t),
$$

where $C$ is a uniform constant, $\eta(t) \rightarrow 0$ as $t \rightarrow 0$ and $\rho$ is defined by (0.7).

For each $t \in\left[0, T\left[\right.\right.$, let $H_{t}$ be the unique continuous harmonic function in $\Omega$ with boundary value $h_{t}$. Then

$$
u \leq U^{*} \leq H .
$$

It follows moreover from Theorem 3.12 that $U^{*}(t, \cdot)$ converges in $L^{1}(\Omega)$ to $h_{0}$ as $t \rightarrow 0$. Hartog's lemma thus yields

$$
\limsup _{t \rightarrow 0} \max _{z \in K}\left(U^{*}(t, z)-h_{0}(z)\right) \leq 0,
$$

for any compact $K \Subset \Omega$. Since $u_{t}$ uniformly converges to $h_{0}$ as $t \rightarrow 0^{+}$we infer, for any compact $K \Subset \Omega$,

$$
\lim _{t \rightarrow 0^{+}} \sup _{z \in K}\left|U^{*}(t, z)-h_{0}(z)\right|=0 .
$$


Fix $\varepsilon>0$. Since $H_{0}$ and $h_{0}$ are continuous on $\bar{\Omega}$ with $h_{0}=H_{0}$ on $\partial \Omega$, there exists $\delta>0$ small enough such that

$$
\sup _{z \in \Omega_{\delta}}\left|H_{0}(z)-h_{0}(z)\right| \leq \varepsilon
$$

where $\Omega_{\delta}:=\{z \in \bar{\Omega} ; \operatorname{dist}(z, \partial \Omega)<\delta\}$. We also have, for $(t, z) \in[0, T[\times \bar{\Omega}$,

$$
U^{*}(t, z)-h_{0}(z) \leq H_{t}(z)-h_{0}(z) \leq H_{0}(z)-h_{0}(z)+\kappa_{h} t
$$

Using this and the uniform convergence of $u_{t}$ to $h_{0}$ as $t \rightarrow 0$ we obtain

$$
\lim _{t \rightarrow 0} \sup _{z \in \Omega_{\delta}}\left|U^{*}(t, z)-h_{0}(z)\right| \leq \varepsilon .
$$

Using (3.6) we infer

$$
\lim _{t \rightarrow 0} \sup _{z \in \bar{\Omega}}\left|U^{*}(t, z)-h_{0}(z)\right| \leq \varepsilon .
$$

Letting $\varepsilon \rightarrow 0^{+}$yields the conclusion.

For the general case (i.e. $h$ is locally uniformly Lipschitz in $] 0, T[$ with $h_{0}$ continuous on $\bar{\Omega}$ ), we proceed by approximation. Fix $\left.S \in\right] T / 2, T[, \varepsilon>0$ small enough. Proposition 4.1 ensures that $U_{h, g, F, \Omega_{S}}=U_{h, g, F, \Omega_{T}}$ in $\Omega_{S}$. Set

$$
\begin{cases}h^{\epsilon}(t, \zeta):=h(t+\epsilon, \zeta) & \text { if }(t, \zeta) \in[0, S] \times \partial \Omega \\ h^{\epsilon}(0, z)=h_{0}(z)+\psi^{\epsilon}(z) & \text { if } z \in \Omega\end{cases}
$$

where $\psi^{\epsilon}$ is the maximal plurisubharmonic function in $\Omega$ such that $\psi^{\epsilon}(\zeta)=$ $h(\epsilon, \zeta)-h(0, \zeta)$ in $\partial \Omega$. Recall that $\psi^{\epsilon}$ is the upper envelope of all psh functions $\psi$ in $\Omega$ whose boundary values satisfy $\psi^{*} \leq h(\epsilon, \zeta)-h(0, \zeta)$ on $\partial \Omega$.

Since $h^{\epsilon}(0, \cdot)=h(\varepsilon, \cdot) \rightarrow h(0, \cdot)$ uniformly on $\partial \Omega$ as $\epsilon \rightarrow 0$, it follows that $\psi^{\epsilon} \rightarrow 0$ uniformly in $\bar{\Omega}$ as $\epsilon \rightarrow 0$. Therefore $\left\{h^{\epsilon}\right\}$ uniformly converges on $\partial_{0} \Omega_{S}$ to $h$ as $\epsilon \rightarrow 0$. Set $U^{\varepsilon}:=U_{h^{\varepsilon}, g, F, \Omega_{S}}$. Then $\left(U^{\varepsilon}\right)^{*}$ uniformly converges to $U^{*}$ in $\Omega_{S}$. Since $h^{\epsilon}$ is uniformly Lipschitz in $t \in[0, S]$, the previous step (using Theorem 3.12) guarantees that $\left(U^{\varepsilon}\right)^{*}(t, \cdot)$ uniformly converges to $h_{0}$ as $t \rightarrow 0$, hence $U_{t}^{*}$ uniformly converges to $h_{0}$ as $t \rightarrow 0$.

\section{Time Regularity of PARABOLIC ENVElopes}

We establish in this section time regularity of the envelope $U:=U_{h, g, F, \Omega_{T}}$ by using and adapting some classical ideas of pluripotential theory.

We work in $\Omega_{S}$ for each $0<S<T$ and eventually let $S \rightarrow T$. We thus assume $T<+\infty$, the family $\{F(\cdot, z, \cdot) ; z \in \Omega\}$ is uniformly Lipschitz and semi-convex in $[0, T] \times J$ for each $J \Subset \mathbb{R}$, and $h$ satisfies

$$
\left.t\left|\partial_{t} h(t, z)\right| \leq \kappa_{h}, \text { for all }(t, z) \in\right] 0, T[\times \partial \Omega,
$$

for some positive constant $\kappa$. The condition (4.1) is equivalent to the fact that for all $(t, z) \in \Omega_{T}$ and $s>0$ with $s t<T$, we have

$$
|h(t, z)-h(s t, z)| \leq \kappa_{h} \frac{|s-1|}{\min (s, 1)} .
$$


If $h$ is uniformly Lipschitz in $t \in[0, T[$ (as in (3.3)) then the above condition is automatically satisfied. On the other hand the condition above implies that $h(\cdot, z), z \in \Omega$ is locally uniformly Lipschitz in $] 0, T[$.

4.1. Lipschitz control in the time variable. The following identity principle plays a crucial role in the sequel. For simplicity we will denote the restriction of $h$ on $\partial_{0} \Omega_{S}$, for $0<S<T$, by $h$.

Proposition 4.1. For all $S \in] 0, T\left[\right.$ we have $U_{h, g, F, \Omega_{T}}=U_{h, g, F, \Omega_{S}}$ in $\Omega_{S}$.

Proof. Set $V:=U_{h, g, F, \Omega_{S}}$ and $U:=U_{h, g, F, \Omega_{T}}$. Fix $u \in \mathcal{S}_{h, g, F}\left(\Omega_{S}\right)$ and $\left.t_{0} \in\right] 0, S[$ such that

$$
\left(d d^{c} u\left(t_{0}, \cdot\right)\right)^{n} \geq e^{\partial_{t} u\left(t_{0}, \cdot\right)+F\left(t_{0}, \cdot, u\left(t_{0}, \cdot\right)\right.} g d V .
$$

Set $M_{1}:=\sup _{\Omega}\left|\partial_{t} u\left(t_{0}, \cdot\right)\right|<+\infty$. If $A \geq M_{1}$ the function

$$
\Omega_{T} \ni(t, z) \mapsto v(t, z):=\left\{\begin{array}{l}
u(t, z), \text { if } t \in\left[0, t_{0}\right], \\
u\left(t_{0}, z\right)-A\left(t-t_{0}\right) \text { if } t \in\left[t_{0}, T[\right.
\end{array}\right.
$$

is again a subsolution to (CMAF) in $\Omega_{T}$. Applying (3.3) on the interval $J:=\left[t_{0}, T\left[\right.\right.$, we obtain that $v^{*} \leq h$ on $\partial_{0} \Omega_{T}$ if $A \geq \kappa_{J}(h)$.

We therefore choose $A \geq \max \left\{M_{1}, \kappa_{J}(h)\right\}$. Then $v \in \mathcal{S}_{h, g, F}\left(\Omega_{T}\right)$ hence $v \leq U$ in $\Omega_{T}$. In particular $u \leq U$ on $\Omega_{t_{0}}$. Taking supremum over all candidates $u$ we obtain $V \leq U$ in $\Omega_{t_{0}}$. Using Proposition 3.2 we can let $t_{0} \rightarrow S$ to obtain $V \leq U$ in $\Omega_{S}$. The reverse inequality is clear.

Theorem 4.2. If $h$ satisfies (4.1), then the envelope $U:=U_{h, g, F, \Omega_{T}}$ satisfies

$$
t\left|\partial_{t} U(t, z)\right| \leq \kappa_{U}, \forall(t, z) \in \Omega_{T},
$$

where $\kappa_{U}>0$ is a uniform constant.

We will show that the constant $\kappa_{U}$ is actually explicit,

$$
\kappa_{U}=(T+1)\left(3 M_{U}+2 \kappa_{h}+2 n+\kappa_{F}\left(T+M_{U}\right)\right) .
$$

This quantitative information will be crucial in perturbation arguments, to obtain uniform Lipschitz constants of the approximants.

The proof of this theorem follows and adapt ideas developed by Bedford and Taylor in their study of Dirichlet problems for elliptic complex MongeAmpère equations (see [BT76, Theorem 6.7], [Dem91]).

Proof. By the assumption on $F$, there exists a constant $\kappa_{F}$ such that, for all $z \in \Omega$ and $\left(t_{j}, r_{j}\right) \in\left[0, T\left[\times\left[-2 M_{U}, 2 M_{U}\right], j=1,2\right.\right.$,

$$
\left|F\left(t_{1}, z, r_{1}\right)-F\left(t_{2}, z, r_{2}\right)\right| \leq \kappa_{F}\left(\left|t_{1}-t_{2}\right|+\left|r_{1}-r_{2}\right|\right) .
$$

Fix $u \in \mathcal{S}_{h, g, F}\left(\Omega_{T}\right)$ such that $\sup _{\Omega_{T}}|u| \leq M_{U}$, where $M_{U}$ is defined in Lemma 3.6. Fix $0<S<T$ and $s \geq 1 / 2$ close to 1 enough such that $s S<T$. Set, for $(t, z) \in \Omega_{S}$,

$$
v^{s}(t, z):=s^{-1} u(s t, z)-C|s-1|(t+1),
$$


where

$$
C:=2 M_{U}+2 \kappa_{h}+2 n+\kappa_{F}\left(T+M_{U}\right) .
$$

We are going to prove that $v^{s} \in \mathcal{S}_{h, g, F}\left(\Omega_{S}\right)$. Since $u$ is a subsolution to (CMAF), for a.e. $t \in] 0, S[$ we have

$$
\begin{aligned}
\left(d d^{c} v^{s}(t, \cdot)\right)^{n} & =s^{-n}\left(d d^{c} u(s t, \cdot)^{n}\right. \\
& \geq e^{-n \log s+\partial_{\tau} u(s t, \cdot)+F(s t, \cdot, u(s t, \cdot))} g d V \\
& \geq e^{\partial_{t} v^{s}(t, \cdot)+C|s-1|+F\left(t, \cdot, s^{-1} u(s t, \cdot)\right)-n \log s-\kappa_{F}\left(T|s-1|+\left|s^{-1}-1\right| M_{U}\right)} g d V \\
& \geq e^{\partial_{t} v^{s}(t, \cdot)+F\left(t, \cdot, v^{s}(t, \cdot)\right)} g d V,
\end{aligned}
$$

where in the last line we use (4.5) and the fact that $F$ is increasing in $r$.

We now take care of the boundary values. For $t \in[0, S], z \in \partial \Omega$ we have

$$
\begin{aligned}
v^{s}(t, z) & \leq-C|s-1|+\left|s^{-1}-1\right| M_{U}+h(s t, z) \\
& \leq-C|s-1|+2|s-1| M_{U}+h(t, z)+2 \kappa_{h}|s-1| \\
& \leq h(t, z),
\end{aligned}
$$

where in the second line we use (4.2), and in the last line we use again (4.5). For $z \in \Omega$ we similarly get $\left(v^{s}\right)^{*}(0, z) \leq h_{0}(z)$.

The computations above show that $v^{s} \in \mathcal{S}_{h, g, F}\left(\Omega_{S}\right)$. Proposition 4.1 thus yields $v^{s} \leq U$ in $\Omega_{S}$. Taking supremum over $u$ we arrive at

$$
s^{-1} U(s t, z)-C|s-1|(t+1) \leq U(t, z) \text {, for all }(t, z) \in \Omega_{S} .
$$

Letting $s \rightarrow 1$ we infer, for all $(t, z) \in \Omega_{S}$,

$$
\left|\partial_{t} U(t, z)\right| \leq M_{U}+C(T+1) .
$$

Letting $S \rightarrow T$ yields the conclusion.

Definition 4.3. Given a constant $\kappa>0$ we let $\mathcal{S}^{\kappa}:=\mathcal{S}_{h, g, F}^{\kappa}\left(\Omega_{T}\right)$ denote the set of all $u \in \mathcal{S}_{h, g, F}\left(\Omega_{T}\right)$ such that, for all $\left.t \in\right] 0, T[$,

$$
\sup _{\Omega}\left|\partial_{t} u(t, z)\right| \leq \kappa / \min (t, b),
$$

where $b=\min (1, T / 2)$, and we set

$$
U^{\kappa}:=U_{h, g, F, \Omega_{T}}^{\kappa}:=\sup \left\{u ; u \in \mathcal{S}_{h, g, F}^{\kappa}\left(\Omega_{T}\right)\right\} .
$$

We will need the following identity principle :

Proposition 4.4. For all $S \in] T / 2, T\left[\right.$ and $\kappa \geq 2 T \kappa_{h}$ we have

$$
U_{h, g, F, \Omega_{T}}^{\kappa}=U_{h, g, F, \Omega_{S}}^{\kappa} \text { in } \Omega_{S} .
$$

Proof. The proof is similar to that of Proposition 4.1. Fix $S \in] T / 2, T[$ and set $V:=U_{h, g, F, \Omega_{S}}^{\kappa}, W:=U_{h, g, F, \Omega_{T}}^{\kappa}$. Fix $u \in \mathcal{S}_{h, g, F}^{\kappa}\left(\Omega_{S}\right)$. Using Proposition 3.2 we fix $\left.t_{0} \in\right] T / 2, S[$ such that

$$
\left(d d^{c} u\left(t_{0}, \cdot\right)\right)^{n} \geq e^{\partial_{t} u\left(t_{0}, \cdot\right)+F\left(t_{0}, \cdot, u\left(t_{0}, \cdot\right)\right.} g d V .
$$


Since $\sup _{\Omega}\left|\partial_{t} u\left(t_{0}, \cdot\right)\right| \leq \kappa / b$, the function

$$
\Omega_{T} \ni(t, z) \mapsto v(t, z):=\left\{\begin{array}{l}
u(t, z), \text { if } t \in\left[0, t_{0}\right], \\
u\left(t_{0}, z\right)-\kappa b^{-1}\left(t-t_{0}\right) \text { if } t \in\left[t_{0}, T[,\right.
\end{array}\right.
$$

is still a subsolution to (CMAF) in $\Omega_{T}$. It follows from (4.2) that

$$
\left|h(t, z)-h\left(t_{0}, z\right)\right| \leq \frac{2 \kappa_{h}}{T}\left|t-t_{0}\right|, \text { for all } t \in\left[t_{0}, T[.\right.
$$

Using that $\kappa \geq 2 T \kappa_{h}$ and $b<1$, we thus obtain $v^{*} \leq h$ on $\partial_{0} \Omega_{T}$. By construction, $v$ satisfies (4.6). Therefore $v \in \mathcal{S}_{h, q, F}^{\kappa}\left(\Omega_{T}\right)$, hence $v \leq W$ in $\Omega_{T}$. We infer in particular $u \leq W$ on $\Omega_{t_{0}}$. Taking supremum over all candidates $u$ we obtain $V \leq W$ in $\Omega_{t_{0}}$. Using Proposition 3.2 we can let $t_{0} \rightarrow S$ to obtain $V \leq W$ in $\Omega_{S}$. The reverse inequality is obvious.

Theorem 4.5. There exists an explicit $\kappa_{0}>0$ such that, for all $\kappa>\kappa_{0}$,

$$
\sup _{\Omega_{T}} t\left|\partial_{t} U^{\kappa}\right| \leq \kappa_{0}
$$

Proof. We use the same notations as in the proof of Theorem 4.2. Define

$$
C:=\kappa_{F} T+2 \kappa_{F} M_{U}+2 M_{F}+2 \kappa_{h}+2 M_{h}+2 n,
$$

and

$$
\kappa_{0}:=2 M_{U}+3 C(T+1)+2 \sup _{\Omega}|\rho| .
$$

Fix $\kappa>\kappa_{0}$. By definition of $\kappa_{0}$ we have, for all $\left.t \in\right] 0, T\left[, 2 \kappa_{h} \leq \kappa_{0} / t\right.$. Proposition 4.4 thus ensures that, for all $T / 2<S<T$,

$$
U_{h, g, F, \Omega_{T}}^{\kappa}=U_{h, g, F, \Omega_{S}}^{\kappa} \text { in } \Omega_{S} .
$$

Fix $u \in \mathcal{S}^{\kappa}, T / 2<S<T, s>0$ close enough to 1 and set, for $(t, z) \in \Omega_{S}$,

$$
w(t, z):=a s^{-1} u(s t, z)+(1-a) \rho-C(1-a)(t+1),
$$

where $a=1-2|s-1|>0, \rho$ is defined in (0.7).

Since $u$ is a subsolution to (CMAF) we have, for almost all $t \in] 0, T$,

$$
\left(d d^{c} s^{-1} u(s t, \cdot)\right)^{n} \geq \exp \left\{-n \log s+\partial_{\tau} u(s t, z)+F(s t, z, u(s t, z))\right\} g d V .
$$

It thus follows from Lemma 2.10 that

$$
\begin{aligned}
\left(d d^{c} w\right)^{n} & \geq \exp \left\{a \partial_{t} u(s t, z)+a F(s t, z, u(s t, z))-a n \log s\right\} g d V \\
& =\exp \left\{\partial_{t} w(t, z)+C(1-a)-a n \log s+a F(s t, z, u(s t, z))\right\} g d V .
\end{aligned}
$$

From (4.4) and the assumption that $F$ is increasing in $r$ we obtain

$$
\begin{aligned}
a F(s t, z, u(s t, z)) & =F(s t, z, u(s t, z))+(1-a) F(s t, z, u(s t, z)) \\
& \geq F\left(t, z, a s^{-1} u(s t, z)\right)-|s-1|\left(\kappa_{F} T+2 \kappa_{F} M_{U}+2 M_{F}\right) \\
& \geq F(t, z, w(t, z))-|s-1|\left(\kappa_{F} T+2 \kappa_{F} M_{U}+2 M_{F}\right) .
\end{aligned}
$$


For $(\tau, \zeta) \in \partial_{0} \Omega_{S}$ we have

$$
\begin{aligned}
w(\tau, \zeta) & \leq a s^{-1} h(s t, \zeta)-2 C|s-1| \\
& \leq h(s t, \zeta)+\left|a s^{-1}-1\right| M_{h}-2 C|s-1| \\
& \leq h(t, \zeta)+2|s-1| \kappa_{h}+2 M_{h}|s-1|-2 C|s-1| .
\end{aligned}
$$

The choice of $C$ in (4.7) and the previous computations ensure that $w \in$ $\mathcal{S}_{h, g, F}\left(\Omega_{S}\right)$. Moreover, for $\left.s \in[1 / 2,3 / 2], t \in\right] 0, S[$,

$$
\sup _{\Omega}\left|\partial_{t} w(t, z)\right| \leq \frac{(1-2|s-1|) \kappa}{\min (s t, b)}+2 C|s-1| .
$$

Since $\kappa / t>\kappa_{0} / t>3 C$, it follows that for $\left.s \in[1,3 / 2], t \in\right] 0, S[$,

$$
\sup _{\Omega}\left|\partial_{t} w(t, z)\right| \leq \frac{(3-2 s) \kappa}{\min (t, b)}+\frac{2(s-1) \kappa}{3 t} \leq \frac{\kappa}{\min (t, b)} .
$$

Hence $w \in \mathcal{S}_{h, g, F}^{\kappa}\left(\Omega_{S}\right)$. By definition of $U^{\kappa}$ and (4.9) we have $w \leq U^{\kappa}$ on $\Omega_{S}$. Taking supremum over $u \in \mathcal{S}_{h, g, F}^{\kappa}\left(\Omega_{T}\right)$ we obtain, for all $(t, z) \in \Omega_{S}$,

$$
a s^{-1} U^{\kappa}(s t, z)-2 C|s-1|(t+1)+2|s-1| \rho(z) \leq U^{\kappa}(t, z) .
$$

Letting $s \rightarrow 1$ yields

$$
t\left|\partial_{t} U^{\kappa}(t, z)\right| \leq 2 M_{U}+2 C(T+1)+2 \sup _{\Omega}|\rho| \leq \kappa_{0},
$$

where in the last inequality we use (4.8). This concludes the proof.

4.2. The maximal subsolution. We now prove that $U \in \mathcal{S}_{h, g, F}\left(\Omega_{T}\right)$.

Theorem 4.6. Assume $h$ satisfies (4.1) and set $U:=U_{h, g, F, \Omega_{T}}$. Then $U \in \mathcal{S}_{h, g, F}\left(\Omega_{T}\right)$ and satisfies the following properties:

(1) $\lim _{\Omega_{T} \ni(t, z) \rightarrow(s, \zeta)} U(t, z)=h(s, \zeta)$ for all $(s, \zeta) \in[0, T[\times \partial \Omega$;

(2) $\lim \sup _{\Omega_{T} \ni(t, z) \rightarrow\left(0, z_{0}\right)} U(t, z)=h\left(0, z_{0}\right)$ for all $\left(0, z_{0}\right) \in\{0\} \times \Omega$;

(3) $\lim _{t \rightarrow 0} U_{t}(z)=h_{0}(z)$ for all $z \in \Omega$.

If $h_{0}$ is continuous then for all $(s, \zeta) \in \partial_{0} \Omega_{T}$,

$$
\lim _{\Omega_{T} \ni(t, z) \rightarrow(s, \zeta)} U(t, z)=h(s, \zeta) .
$$

Proof. We proceed in several steps.

Step 1. Assume $h$ satisfies (3.3).

Theorem 3.12 ensures that $U^{*}$ has the desired boundary values. We are going to prove that $U^{*}$ is a subsolution to (CMAF).

Step 1.1. Assume $h_{0}$ is continuous on $\bar{\Omega}$.

Fix $\kappa \geq \kappa_{0}$, where $\kappa_{0}$ is defined in Theorem 4.5.

Claim 1: $U^{\kappa}=\left(U^{\kappa}\right)^{*} \in \mathcal{S}_{h, g, F}^{\kappa}\left(\Omega_{T}\right)$.

Indeed, since $U^{\kappa} \leq U$, the boundary condition $\left.\left(U^{\kappa}\right)^{*}\right|_{\partial_{0} \Omega_{T}} \leq h$ is satisfied. We now prove that $\left(U^{\kappa}\right)^{*}$ is a subsolution to (CMAF). A classical lemma 
of Choquet ensures that there exists a sequence $\left\{u^{j}\right\}$ in $\mathcal{S}^{\kappa}\left(h, g, F, \Omega_{T}\right)$ such that

$$
\left(U^{\kappa}\right)^{*}=\left(\sup _{j \in \mathbb{N}} u^{j}\right)^{*} \text { in } \Omega_{T} .
$$

By Lemma 3.6, we can assume $\sup _{\Omega_{T}}\left|u^{j}\right| \leq M_{U}$. Since $\mathcal{S}^{\kappa}$ is stable under taking maximum we can assume that $\left\{u^{j}\right\}$ is increasing. By definition of $\mathcal{S}^{\kappa}, \lim _{j} u^{j}$ is locally uniformly Lipschitz in $\left.t \in\right] 0, T$. Hence from Lemma 1.4 it follows that $u^{j}$ increases to $\left(U^{\kappa}\right)^{*}$ almost everywhere in $\Omega_{T}$. We infer that $d t \wedge\left(d d^{c} u_{j}\right)^{n} \rightarrow d t \wedge\left(d d^{c}\left(U^{\kappa}\right)^{*}\right)^{n}$ weakly in $\Omega_{T}$. Moreover, the sequence $\left\{\psi_{j}\right\}:=\left\{\partial_{t} u^{j}+F\left(t, z, u^{j}\right)\right\}$ is bounded and converges in the sense of distributions to $\partial_{t}\left(U^{\kappa}\right)^{*}+F\left(t, z,\left(U^{\kappa}\right)^{*}\right)$. Proposition 2.6 thus yields

$$
e^{\partial_{t}\left(U^{\kappa}\right)^{*}+F\left(t, z,\left(U^{\kappa}\right)^{*}\right)} g d t \wedge d V \leq \liminf _{j} e^{\partial_{t} u^{j}+F\left(t, z, u^{j}\right)} g d t \wedge d V
$$

weakly in $\Omega_{T}$. Therefore, $\left(U^{\kappa}\right)^{*}$ is a subsolution to $(\mathrm{CMAF})$ in $\Omega_{T}$. Hence $\left(U^{\kappa}\right)^{*}=U^{\kappa}$ and Claim 1 is proved.

It now follows from Theorem 4.5 that $U^{\kappa}=U^{\kappa_{0}}$, for all $\kappa>\kappa_{0}$.

Claim 2: $U=U^{\kappa_{0}}$ in $\Omega_{T}$.

Fix $\left.v \in \mathcal{S}_{h, g, F}\left(\Omega_{T}\right), S \in\right] T / 2, T[, \varepsilon>0$ small enough. Define, for $(t, z) \in$ $[0, S] \times \Omega$,

$$
u(t, z):=v(t+\varepsilon, z)-C \varepsilon(1+t)-\theta(\varepsilon),
$$

where $C>0$ is a uniform constant and $\theta(\varepsilon):=\sup _{\bar{\Omega}}\left|U^{*}(\varepsilon, z)-h_{0}(z)\right|$ converges to 0 (by Lemma 3.13). Since $v^{*} \leq h$ on $\partial_{0} \Omega_{T}$, we obtain for all $(\tau, \zeta) \in[0, S] \times \partial \Omega$,

$$
u(\tau, \zeta) \leq h(\tau+\varepsilon, \zeta)-C \varepsilon \leq h(\tau, \zeta),
$$

if $C \geq \kappa_{h}$. By definition of $\theta(\varepsilon)$ we also have $u(0, z) \leq h_{0}(z)$ in $\Omega$.

A direct computation shows that, for $C>0$ large enough, $u \in \mathcal{S}_{h, g, F}\left(\Omega_{S}\right)$. Since $u$ is uniformly Lipchitz in $[0, S], u \in \mathcal{S}_{h, g, F}^{\kappa}\left(\Omega_{S}\right)$ for some $\kappa>0$ large enough. Hence $u \leq U^{\kappa_{0}}$ in $\Omega_{S}$. Letting $\varepsilon \rightarrow 0$ we obtain $v \leq U^{\kappa_{0}}$ in $\Omega_{S}$. Letting $S \rightarrow T$ we arrive at $v \leq U^{\kappa_{0}}$, hence $U \leq U^{\kappa_{0}}$. Therefore $U=U^{\kappa_{0}}$ is the maximal subsolution to (CMAF) with boundary value $h$.

Step 1.2. We now remove the continuity assumption on $h_{0}$.

Using Lemma 2.11 we can find a sequence $h_{0}^{j}$ of psh functions in $\Omega$ such that $h_{0}^{j}$ is continuous on $\bar{\Omega}, h_{0}^{j}=h_{0}$ on $\partial \Omega$, and $h_{0}^{j} \downarrow h_{0}$ in $\Omega$. We then define $h^{j}(t, z):=h(t, z)$ for $(t, z) \in\left[0, T\left[\times \partial \Omega\right.\right.$ and $h^{j}(0, z)=h_{0}^{j}(z)$ for $z \in \Omega$. We thus obtain a sequence of continuous Cauchy-Dirichlet boundary data for $\Omega_{T}$ such that $h^{j}=h$ on $\left[0, T\left[\times \partial \Omega\right.\right.$ and $h^{j}$ decreases pointwise to $h$. The previous step ensures that $U^{j}:=U_{h^{j}, g, F, \Omega_{T}}$ is a subsolution to (CMAF). Theorem 4.2 and Theorem 4.7 provide a uniform Lipschitz constant for $U^{j}$. Since $h^{j}$ decreases to $h, U \leq U^{j}$ decreases to some $V \in \mathcal{P}\left(\Omega_{T}\right)$. We thus have $\left.V^{*}\right|_{\partial_{0} \Omega_{T}} \leq h$, and Proposition 2.6 reveals that $V$ is a subsolution to (CMAF). It then follows that $V=U$. 
Step 2. To treat the general case we proceed by approximation as in the proof of Lemma 3.13. Fix $0<S<T$ and $0<\varepsilon<(T-S) / 2$. Define

$$
\begin{cases}h^{\epsilon}(t, \zeta):=h(t+\epsilon, \zeta) & \text { if }(t, \zeta) \in[0, S] \times \partial \Omega \\ h^{\epsilon}(0, z)=h_{0}(z)+\psi^{\epsilon}(z) & \text { if } z \in \Omega,\end{cases}
$$

where $\psi^{\epsilon}$ is the maximal psh function in $\Omega$ such that $\psi^{\epsilon}(\zeta)=h(\epsilon, \zeta)-h(0, \zeta)$ in $\partial \Omega$. Then $\left\{h^{\epsilon}\right\}$ uniformly converges on $\partial_{0} \Omega_{S}$ to $h$ as $\epsilon \rightarrow 0$. Since $h^{\epsilon}$ is uniformly Lipschitz in $t \in[0, S]$, the previous step and Theorem 3.12 ensure that $U^{\varepsilon}:=U_{h^{\varepsilon}, g, F, \Omega_{S}} \in \mathcal{S}_{h^{\varepsilon}, F, g}\left(\Omega_{S}\right)$ satisfies the boundary conditions (1), (2), (3). Moreover, it follows Proposition 4.1 that the envelopes $U^{\varepsilon}$ uniformly converge in $\Omega_{S}$ to $U$ as $\varepsilon \rightarrow 0$. Hence, Proposition 2.6 and Proposition 2.3 (together with Remark 2.4) yield that $U$ is a subsolution to (CMAF) and $U$ satisfies the boundary conditions (1), (2), (3).

If $h_{0}$ is continuous on $\bar{\Omega}$ then Lemma 3.13 and the three boundary conditions (1), (2), (3) give the last statement.

4.3. Semi-concavity in the time variable. In this section we assume that $h$ satisfies (4.1) and there exists $C_{h}>0$ such that, for all $z \in \partial \Omega$,

$$
\partial_{t}^{2} h(t, z) \leq C_{h} t^{-2}
$$

in the sense of distributions in $] 0, T$. Condition (4.10) is equivalent to the fact that $t \mapsto h(t, z)+C_{h} \log t$ is concave in $] 0, T$ [. It implies in particular that $h$ is locally uniformly semi-concave in the $t$-variable.

Theorem 4.7. Assume $h$ satisfies (4.1) and (4.10). The envelope $U:=$ $U_{h, g, F, \Omega_{T}}$ is locally uniformly semi-concave in $] 0, T[$ : for all $z \in \Omega$,

$$
\partial_{t}^{2} U(t, z) \leq C_{U} t^{-2}
$$

in the sense of distributions in $] 0, T\left[\right.$, for some uniform constant $C_{U}>0$.

We will show that the constant $C_{U}$ is actually explicit,

$$
C_{U}:=C_{h}+2 M_{h}+8 \kappa_{h}+\left(2 \kappa_{F}+3\right)\left(M_{U}+5 \kappa_{U}+1+C_{F} T^{2}+16 \kappa_{U}^{2}\right) .
$$

This quantitative information is important in perturbation arguments, to obtain uniform semi-concavity constants of the approximants.

By the assumption on $F$, there is a constant $C_{F}>0$ such that for all $z \in \Omega$, the function

$$
(t, r) \mapsto F(t, z, r)+C_{F}\left(t^{2}+r^{2}\right) \text { is convex in }[0, T] \times\left[-2 M_{U}, 2 M_{U}\right] .
$$

Proof. It follows from Theorem 4.6 that $U \in \mathcal{S}_{h, g, F}\left(\Omega_{T}\right)$. Fix $0<S<T$, and $s>1 / 2$ close to 1 enough such that $s S<T$. Set, for $(t, z) \in \Omega_{S}$,

$$
v^{s}(t, z):=\frac{s^{-1} U(s t, z)+s U\left(s^{-1} t, z\right)}{2}-C(t+1)(s-1)^{2},
$$

where $C>0$ is defined as

$$
C:=C_{h}+1+2 M_{h}+8 \kappa_{h}+2 \kappa_{F}\left(M_{U}+4 \kappa_{U}+T+C_{F} T^{2}+16 \kappa_{U}^{2}\right) .
$$

We are going to prove that $v^{s} \in \mathcal{S}_{h, g, F}\left(\Omega_{S}\right)$. 
Boundary values of $v^{s}$. It follows from (4.10) that for all $\left.z \in \partial \Omega, t \in\right] 0, S[$,

$$
\begin{aligned}
\frac{h(s t, z)+h\left(s^{-1} t, z\right)}{2} & \leq h\left(\frac{\left(s+s^{-1}\right) t}{2}, z\right)+C_{h} \log \left(\frac{s+s^{-1}}{2}\right) \\
& \leq h\left(\frac{\left(s+s^{-1}\right) t}{2}, z\right)+C_{h}(s-1)^{2} \\
& \leq h(t, z)+\left(C_{h}+1\right)(s-1)^{2},
\end{aligned}
$$

where in the last line we use (4.2). We claim that for all $(t, z) \in] 0, S[\times \partial \Omega$,

$$
s^{-1} h(s t, z)+\operatorname{sh}\left(s^{-1} t, z\right) \leq h(s t, z)+h\left(s^{-1} t, z\right)+\left(2 M_{h}+3 \kappa_{h}\right)(s-1)^{2} .
$$

Indeed, write $s=1-\sigma$ and observe that $s^{-1}=1+\sigma+O\left(\sigma^{2}\right)$, where $\left|O\left(\sigma^{2}\right)\right| \leq 2 \sigma^{2}$ for $|\sigma| \leq 1 / 2$. Thus for all $\left.(t, z) \in\right] 0, S[\times \partial \Omega$,

$$
\begin{aligned}
s^{-1} h(s t, z) & +s h\left(s^{-1} t, z\right) \leq(1+\sigma) h(s t, z)+(1-\sigma) h\left(s^{-1} t, z\right)+2 M_{h} \sigma^{2} \\
& \leq h(s t, z)+h\left(s^{-1} t, z\right)+\sigma\left(h(s t, z)-h\left(s^{-1} t, z\right)\right)+2 M_{h} \sigma^{2} .
\end{aligned}
$$

Using (4.2), we obtain

$$
s^{-1} h(s t, z)+\operatorname{sh}\left(s^{-1} t, z\right) \leq h(s t, z)+h\left(s^{-1} t, z\right)+\left(2 M_{h}+4 \kappa_{h}\right)(s-1)^{2},
$$

which proves the claim.

Since $\left.U^{*}\right|_{\partial_{0} \Omega_{T}} \leq h$, the above estimate implies that $\left(v^{s}\right)^{*} \leq h$ on $\partial_{0} \Omega_{S}$. Using similarly the estimate in Theorem 4.2 , we obtain the following estimate which will be useful later: for all $(t, z) \in] 0, S[\times \bar{\Omega}$,

$$
\begin{aligned}
\mid\left(U(s t, z)+U\left(s^{-1} t, z\right)\right) & -\left(s^{-1} U(s t, z)+s U\left(s^{-1} t, z\right)\right) \mid \\
\leq & \left(2 M_{U}+4 \kappa_{U}\right)(s-1)^{2} .
\end{aligned}
$$

Estimating the Monge-Ampère measure of $v^{s}$. It follows from Proposition 3.2 that for almost all $t \in] 0, S[$,

$$
\left(d d^{c} s^{-1} U(s t, \cdot)\right)^{n} \geq e^{n \log s^{-1}+\partial_{\tau} U(s t, \cdot)+F(s t, \cdot, U(s t, \cdot))} g d V .
$$

Using Lemma 2.10 we infer

$$
\left(d d^{c} v^{s}(t, \cdot)\right)^{n} \geq e^{a(s)+a\left(s^{-1}\right)} g d V
$$

where

$$
a(s)=\frac{1}{2}\left(\partial_{\tau} U(s t, \cdot)+F(s t, \cdot, U(s t, \cdot))\right) .
$$

By the semi-convexity assumption (4.12) on $F$, for $\lambda \in] 0,1\left[, t_{1}, t_{2} \in[0, T]\right.$, $r_{1}, r_{2} \in\left[-2 M_{U}, 2 M_{U}\right]$ we have

$$
\begin{aligned}
F\left(\lambda\left(t_{1}, r_{1}\right)+(1-\lambda)\left(t_{2}, r_{2}\right)\right) & \leq \lambda F\left(t_{1}, r_{1}\right)+(1-\lambda) F\left(t_{2}, r_{2}\right) \\
& +C_{F} \lambda(1-\lambda)\left(\left(t_{1}-t_{2}\right)^{2}+\left(r_{1}-r_{2}\right)^{2}\right) .
\end{aligned}
$$


Applying this for $(t, r) \mapsto F(t, z, r), z \in \Omega, \lambda=1 / 2, t_{1}=s t, t_{2}=s^{-1} t$, $r_{1}=U(s t, z), r_{2}=U\left(s^{-1} t, z\right)$, we obtain

$$
\begin{aligned}
& \quad \frac{1}{2} F(s t, z, U(s t, z))+\frac{1}{2} F\left(s^{-1} t, z, U\left(s^{-1} t, z\right)\right) \geq \\
& F\left(\frac{\left(s+s^{-1}\right) t}{2}, z,\left(U(s t, z)+U\left(s^{-1} t, z\right)\right) / 2\right) \\
& -\frac{C_{F}}{4}\left(t^{2}\left(s-s^{-1}\right)^{2}+\left(U(s t, \cdot)-U\left(s^{-1} t, \cdot\right)\right)^{2}\right) .
\end{aligned}
$$

Using (4.4), (4.14), and the fact that $F$ is increasing in $r$, we thus get

$$
\begin{aligned}
& \quad \frac{1}{2} F(s t, z, U(s t, z))+\frac{1}{2} F\left(s^{-1} t, z, U\left(s^{-1} t, z\right)\right) \geq \\
& F\left(t, z, v^{s}(t, z)\right)-\kappa_{F}\left(M_{U}+2 \kappa_{U}+t\right)(s-1)^{2} \\
& -\frac{C_{F}}{4}\left(t^{2}\left(s-s^{-1}\right)^{2}+\left(U(s t, \cdot)-U\left(s^{-1} t, \cdot\right)\right)^{2}\right) \\
& \geq F\left(t, z, v^{s}(t, z)\right)-\left(\kappa_{F}\left(M_{U}+2 \kappa_{U}+T\right)+2 C_{F}\left(T^{2}+2 \kappa_{U}^{2}\right)\right)(s-1)^{2} .
\end{aligned}
$$

The choice of $C$ (4.13) ensures that

$$
a(s)+a\left(s^{-1}\right) \geq \partial_{t} v^{s}(t, \cdot)+F\left(t, \cdot, v^{s}(t, \cdot)\right) .
$$

Altogether we conclude that $v^{s} \in \mathcal{S}_{h, g, F}\left(\Omega_{S}\right)$. Using Proposition 4.1 we infer $v^{s} \leq U$ in $\Omega_{S}$. From this and (4.14) we obtain that for all $(t, z) \in \Omega_{S}$,

$$
\frac{U(s t, z)+U\left(s^{-1} t, z\right)}{2}-U(t, z) \leq\left(C+2 M_{U}+8 \kappa_{U}\right)(s-1)^{2} .
$$

An elementary computation then yields (letting $s \rightarrow 1$ ) that $\forall(t, z) \in \Omega_{S}$,

$$
t^{2} \partial_{t}^{2} U(t, z) \leq\left(9 \kappa_{U}+2 M_{U}+C\right) .
$$

We finally let $S \rightarrow T$ to conclude the proof.

\section{Space Regularity of Parabolic envelopes}

We establish the first steps of a balayage process by studying solutions constructed in small balls, and establishing space regularity of $U_{h, g, F, \mathbb{B}_{T}}$ : assuming adequate regularity conditions on the data we prove that $U_{h, g, F, \mathbb{B}_{T}}$ is locally $\mathcal{C}^{1,1}$ in $z \in \mathbb{B}$.

We assume that $T<+\infty$, and $h$ satisfies (4.1) and (4.10).

5.1. Continuity in the space variable. Let $\left(Y, d_{Y}\right)$ be a metric space. The uniform partial modulus of continuity in the space variable $y \in Y$ of a function $u:[0, T[\times Y \longrightarrow \mathbb{R}$ is

$$
\eta(u, \delta):=\sup \left\{\left|u\left(t, y_{1}\right)-u\left(t, y_{2}\right)\right| ; t \in\left[0, T\left[, y_{1}, y_{2} \in Y, d_{Y}\left(y_{1}, y_{2}\right) \leq \delta\right\} .\right.\right.
$$

In particular, the uniform partial modulus of continuity of $F$ is defined as above with $Y:=\Omega \times \mathbb{R}$.

Theorem 5.1. Assume the following conditions : 
- $G:=\log g$ is continuous in $\Omega$;

- there exists $u \in \mathcal{S}_{h, g, F}\left(\Omega_{T}\right) \cap \mathcal{C}\left(\left[0, T[\times \bar{\Omega})\right.\right.$, such that $u=h$ on $\partial_{0} \Omega_{T}$.

Then $U:=U_{h, g, F, \Omega_{T}}$ is continuous on $[0, T[\times \bar{\Omega}$ and

$$
\eta(U, \delta) \leq \eta(u, \delta)+\eta(H, \delta)+(\eta(F, \delta)+\eta(G, \delta)) T .
$$

Recall that $H_{t}$ is the unique harmonic function in $\Omega$ with $H_{t}=h_{t}$ on $\partial \Omega$.

A continuous subsolution which agrees with $h$ on $\partial_{0} \Omega$ is called a subbarrier. Such a subbarrier (for the whole boundary $\partial_{0} \Omega_{T}$ as required in the Theorem) exists when $h$ is uniformly Lipschitz in [0,T[ and continuous on $\partial_{0} \Omega_{T}$ by Lemma 3.7, Lemma 3.8 and Lemma 3.9.

Proof. It follows from Theorem 3.12 that $U$ continuously extends to the boundary $\partial_{0} \Omega_{T}$ so that $U=h$ on $\partial_{0} \Omega_{T}$. We use the perturbation method of Walsh [Wal69] to extend this property to the interior and prove that $U$ is continuous on $[0, T[\times \bar{\Omega}$.

Fix $\delta>0$ small enough. Since $u=h=U$ in $[0, T[\times \partial \Omega$, we infer that for all $t \in[0, T[, z \in \Omega, \zeta \in \partial \Omega$ with $|z-\zeta| \leq \delta$,

$$
U(t, \zeta)=u(t, \zeta) \leq u(t, z)+\eta(u, \delta) \leq U(t, z)+\eta(u, \delta) .
$$

Fix $\xi \in \mathbb{C}^{n}$ such that $|\xi| \leq \delta$ and set $\Omega_{\xi}:=\Omega-\xi$ and consider

$$
W(t, z):=\left\{\begin{array}{l}
U(t, z), \text { if } t \in\left[0, T\left[, z \in \Omega \backslash \Omega_{\xi},\right.\right. \\
\max \{U(t, z), U(t, z+\xi)-\eta(u, \delta)\}, \text { if } t \in\left[0, T\left[, z \in \Omega \cap \Omega_{\xi} .\right.\right.
\end{array}\right.
$$

By (5.2) the two definitions coincide when $(t, z) \in[0, T[\times \Omega$ and $z+\xi \in \partial \Omega$. Therefore $W \in \mathcal{P}\left(\Omega_{T}\right)$. We are going to prove that $W-O(\delta)(t+1) \in$ $\mathcal{S}_{h, g, F}\left(\Omega_{T}\right)$ for some small error term $O(\delta)$.

The subsolution property. By Lemma 3.4, for a.e. $(t, z) \in\left[0, T\left[\times\left(\Omega \cap \Omega_{\xi}\right)\right.\right.$,

$$
\partial_{t} W(t, z)=\mathbb{1}_{\{U(t, z)<\tilde{U}(t, z)\}} \partial_{t} \tilde{U}(t, z)+\mathbb{1}_{\{U(t, z) \geq \tilde{U}(t, z)\}} \partial_{t} U(t, z),
$$

where

$$
\tilde{U}(t, z):=U(t, z+\xi)-\eta(u, \delta) \text { in } \Omega \cap \Omega_{\xi} .
$$

Moreover

$$
\begin{aligned}
& e^{\partial_{t} \tilde{U}(t, z)+F(t, z, \tilde{U}(t, z))+G(z)} d t \wedge d V(z) \\
& \leq e^{\partial_{t} U(t, z+\xi)+F(t, z+\xi, U(t, z+\xi))+\eta(F, \delta)+G(z+\xi)+\eta(G, \delta)} d t \wedge d V(z) \\
& \leq e^{\eta(F, \delta)+\eta(G, \delta)} d t \wedge\left(d d^{c} \tilde{U}\right)^{n},
\end{aligned}
$$

in the weak sense on $] 0, T\left[\times\left(\Omega \cap \Omega_{\xi}\right)\right.$. We thus obtain

$$
e^{\partial_{t} W(t, z)+F(t, z, W(t, z))+G(z)} d t \wedge d V(z) \leq e^{b(\delta)} d t \wedge\left(d d^{c} W\right)^{n},
$$

i.e. the function defined on $\left[0, T\left[\times \Omega\right.\right.$ by $W_{1}(t, z):=W(t, z)-b(\delta) t$, is a subsolution to (CMAF) in $] 0, T[\times \Omega$. Here $b(\delta):=\eta(F, \delta)+\eta(G, \delta)$.

Estimating boundary values. It follows from Theorem 3.12 that

$$
\lim _{\left(t, z^{\prime}\right) \rightarrow(0, z)} U\left(t, z^{\prime}\right)=h_{0}(z), z \in \Omega .
$$


By definition of $W$ and the assumption that $h_{0}=u$ on $\{0\} \times \Omega$, we obtain

$$
\lim _{\left(t, z^{\prime}\right) \rightarrow(0, z)} W\left(t, z^{\prime}\right) \leq h_{0}(z), \text { for all } z \in \Omega .
$$

Fix $(\tau, \zeta) \in[0, T[\times \partial \Omega$.

Since $U \leq H$ in $\Omega_{T}$ and $U=h$ in $[0, T[\times \partial \Omega$, we infer

$\lim _{] 0, T\left[\times\left(\Omega \cap \Omega_{\xi}\right) \ni(t, z) \rightarrow(\tau, \zeta)\right.} W(t, z) \leq \max (h(\tau, \zeta), H(\tau, \zeta+\xi)) \leq h(\tau, \zeta)+\eta(H, \delta)$,

and

$$
\lim _{] 0, T\left[\times\left(\Omega \backslash \Omega_{\xi}\right) \ni(t, z) \rightarrow(\tau, \zeta)\right.} W(t, z)=\lim _{] 0, T\left[\times\left(\Omega \backslash \Omega_{\xi}\right) \ni(t, z) \rightarrow(\tau, \zeta)\right.} U(t, \zeta)=h(\tau, \zeta) .
$$

From the computations above we conclude that $W_{1}-\eta(H, \delta) \in \mathcal{S}_{h, g, F}\left(\Omega_{T}\right)$. Thus $W_{1}-\eta(H, \delta) \leq U$ in $\Omega_{T}$, hence

$$
U(t, z+\xi)-\eta(u, \delta)-\eta(H, \delta)-(\eta(F, \delta)+\eta(G, \delta)) t \leq U(t, z),
$$

for $(t, z) \in\left[0, T\left[\times\left(\Omega \cap \Omega_{\xi}\right)\right.\right.$ and $\xi \in \mathbb{C}^{n}$ with $|\xi| \leq \delta$. This gives (5.1).

The continuity of $U$ on $[0, T[\times \bar{\Omega}$ follows from Theorem 4.6, the continuity of $h_{0}$, the continuity of each slice $U(t, \cdot)$ on $\bar{\Omega}$ and the fact that $U$ is locally uniformly Lipschitz in $t \in] 0, T[$.

Corollary 5.2. Assume that

(1) $G:=\log g$ is Lipschitz in $\Omega$;

(2) the family $\{h(\cdot, z) ; z \in \partial \Omega\}$ is uniformly Lipschitz in $[0, T[$;

(3) $h_{0}$ is Lipschitz on $\bar{\Omega}$;

(4) the family $\{h(t, \cdot) ; t \in] 0, T[\}$ is uniformly $\mathcal{C}^{1,1}$ on $\partial \Omega$;

(5) the function $F$ is Lipschitz on $[0, T[\times \Omega \times J$, for each $J \Subset \mathbb{R}$.

Then the family $\{U(t, \cdot) ; t \in[0, T[\}$ is uniformly Lipschitz on $\bar{\Omega}$.

Proof. It follows from Lemma 3.7, Lemma 3.8 and assumption (2) that there exists $u \in \mathcal{S}_{g, h, F}\left(\Omega_{T}\right) \cap \mathcal{C}\left(\left[0, T[\times \bar{\Omega})\right.\right.$ with $\left.u\right|_{\partial_{0} \Omega_{T}}=h . \quad[\mathrm{GZ}$, Theorem 5.2] and (3), (4) ensure that the family $\{u(t, \cdot) ; t \in[0, T[\}$ is uniformly Lipschitz on $\bar{\Omega}$. We now invoke Theorem 5.1 to finish the proof.

5.2. $\mathcal{C}^{1,1}$-regularity in the space variable. We prove the following regularity result:

Theorem 5.3. Assume $\Omega=\mathbb{B}$ is the unit ball, $T<+\infty$, and

(1) $G:=\log g \in C^{1,1}(\overline{\mathbb{B}})$;

(2) $h$ satisfies the assumptions of Corollary 5.2;

(3) $F$ is Lipschitz and semi-convex in $[0, T[\times \overline{\mathbb{B}} \times J$, for each $J \Subset \mathbb{R}$.

Then the envelope $U_{h, g, F, \mathbb{B}_{T}}$ is locally uniformly $\mathcal{C}^{1,1}$ in $z \in \mathbb{B}$.

By scaling and translating, the result still holds for any ball $B\left(z_{0}, r\right) \Subset \mathbb{C}^{n}$. In the proof below we use $C$ to denote various uniform constants which may be different from place to place. 
Proof. The proof is a parabolic analogue of [BT76, Theorem 6.7]. We follow closely the presentation of [GZ, Theorem 5.3.1]. Recall from Corollary 5.2 that the family $\{U(t, \cdot) ; t \in[0, T[\}$ is uniformly Lipschitz on $\overline{\mathbb{B}}$.

Automorphisms of the ball $\mathbb{B}$. For $a \in \mathbb{B}$, we set

$$
\mathcal{T}_{a}(z)=\frac{P_{a}(z)-a+\sqrt{1-|a|^{2}}\left(z-P_{a}(z)\right)}{1-\langle z, a\rangle} ; P_{a}(z)=\frac{\langle z, a\rangle}{|a|^{2}} a
$$

where $\langle\cdot, \cdot\rangle$ denote the Hermitian product in $\mathbb{C}^{n}$. It is well known (see [Klim, Lemma 4.3.1]) that $\mathcal{T}_{a}$ is a holomorphic automorphism of the unit ball such that $\mathcal{T}_{a}(a)=0$ and $\mathcal{T}_{a}(\partial \mathbb{B})=\partial \mathbb{B}$. Note that $\mathcal{T}_{0}$ is the identity. We set

$$
\xi=\xi(a, z):=a-\langle z, a\rangle z
$$

Observe that $\xi(-a, z)=-\xi(a, z)$. If $|a| \leq 1 / 2$ then

$$
\mathcal{T}_{a}(z)=z-\xi+O\left(|a|^{2}\right)
$$

where $O\left(|a|^{2}\right) \leq C_{0}|a|^{2}$, with $C_{0}$ a numerical constant independent of $z \in \mathbb{B}$ when $|a| \leq 1 / 2$. Thus $\mathcal{T}_{ \pm a}$ is the translation by $\mp \xi$ up to small second order terms, when $|a|$ is small enough.

We set, for $(t, z) \in \mathbb{B}_{T}$,

$$
V_{a}(t, z):=\frac{1}{2}\left(U\left(t, \mathcal{T}_{a}(z)\right)+U\left(t, T_{-a}(z)\right) .\right.
$$

We are going to prove that, for a uniform constant $C>0$, the function $V_{a}-C|a|^{2}(t+1)$ belongs to $\mathcal{S}_{h, g, F}\left(\mathbb{B}_{T}\right)$. We proceed in two steps.

Step 1: Boundary values of $V_{a}$. If $q$ is $\mathcal{C}^{1,1}(\overline{\mathbb{B}})$ then, as in [GZ, Page 145],

$$
\left|q\left(\mathcal{T}_{a}(z)\right)+q\left(\mathcal{T}_{-a}(z)\right)-2 q(z)\right| \leq 2 C(q)|a|^{2},
$$

where $C(q)>0$ depends on the uniform $\mathcal{C}^{1,1}$-norm of $q$ on $\overline{\mathbb{B}}$.

Since the family $\{h(t, \cdot) ; t \in[0, T[\}$ is uniformly Lipschitz in $\partial \mathbb{B}$, applying (5.3) yields

$$
h\left(t, \mathcal{T}_{a}(z)\right)+h\left(t, \mathcal{T}_{-a}(z)\right) \leq 2 h(t, z)+2 C(h)|a|^{2},
$$

for $z \in \partial \mathbb{B},|a|$ small enough, where $C(h)>0$ depends on the uniform $\mathcal{C}^{1,1}$-bound of $h(t, \cdot)$ in a neighborhood of $\partial \mathbb{B}$. We infer, for all $(t, \zeta) \in \partial_{0} \mathbb{B}_{T}$,

$$
V_{a}(t, \zeta) \leq h(t, \zeta)+C(h)|a|^{2} .
$$

Step 2: Estimating the Monge-Ampère measure of $V_{a}$. Since $U$ is a subsolution to (CMAF) a direct computation shows that

$$
\begin{aligned}
& \left(d d^{c} U \circ \mathcal{T}_{a}\right)^{n}=\left|\operatorname{det} \mathcal{T}_{a}^{\prime}\right|^{2}\left(d d^{c} U\right)^{n} \circ \mathcal{T}_{a} \\
& \quad \geq\left|\operatorname{det} \mathcal{T}_{a}^{\prime}\right|^{2} \exp \left(\partial_{t} U\left(t, \mathcal{T}_{a}(z)\right)+F\left(t, \mathcal{T}_{a}(z), U\left(t, \mathcal{T}_{a}(z)\right)+G\left(\mathcal{T}_{a}(z)\right)\right) .\right.
\end{aligned}
$$

Since the function $(a, z) \mapsto \theta(0, z):=\log \left|\operatorname{det} \mathcal{T}_{a}^{\prime}(z)\right|^{2}+\log \left|\operatorname{det} \mathcal{T}_{-a}^{\prime}(z)\right|^{2}$ is smooth in $\mathbb{B}_{1 / 2} \times \overline{\mathbb{B}}$ and $\theta(0, z)=0$, the Taylor expansion yields

$$
\theta(a, z)+\theta(-a, z)=O\left(|a|^{2}\right)
$$


The assumption (3) provides us with a uniform constant $C$ such that

$$
\begin{aligned}
& \frac{1}{2}\left\{F \left(t, \mathcal{T}_{a}(z), U\left(t, \mathcal{T}_{a}(z)\right)+F\left(t, \mathcal{T}_{-a}(z), U\left(t, \mathcal{T}_{-a}(z)\right)\right\}\right.\right. \\
& \geq F\left(t, \frac{\mathcal{T}_{a}(z)+\mathcal{T}_{-a}(z)}{2}, V_{a}(t, z)\right) \\
& -C\left(\left\|\mathcal{T}_{a}(z)-\mathcal{T}_{-a}(z)\right\|^{2}+\left(U\left(t, \mathcal{T}_{a}(z)-U\left(t, \mathcal{T}_{-a}(z)\right)^{2}\right)\right.\right. \\
& \geq F\left(t, z, V_{a}(t, z)\right)-C|a|^{2}
\end{aligned}
$$

where in the last inequality we have used $\mathcal{T}_{a}(z)+\mathcal{T}_{-a}(z)-2 z=O\left(|a|^{2}\right)$, $\mathcal{T}_{a}(z)-\mathcal{T}_{-a}(z)=O(|a|)$, and the Lipschitz regularity (in $z \in \overline{\mathbb{B}}$ ) of $U$. Using this, and applying Lemma 2.10 and the uniform estimate (5.3) to the function $G$ we obtain

$$
\left(d d^{c} V_{a}(t, \cdot)\right)^{n} \geq \exp \left\{\partial_{t} V_{a}+F\left(t, z, V_{a}(t, z)\right)+G(z)-C|a|^{2}\right\} .
$$

By the computations above we conclude that the function

$$
\mathbb{B}_{T} \ni(t, z) \mapsto W_{a}(t, z)=V_{a}(t, z)-C|a|^{2}(t+1)
$$

belongs to $\mathcal{S}_{h, g, F}\left(\mathbb{B}_{T}\right)$. Therefore, for all $(t, z) \in \mathbb{B}_{T}$,

$$
V_{a}(t, z)-(T+1) C|a|^{2} \leq U(t, z) .
$$

From this estimate, we proceed as in [GZ, page 146-147] to prove that the second order partial derivatives (in $z$ ) of $U$ are locally bounded in $\mathbb{B}$.

We now show that $U$ admits a Taylor expansion up to order $(1,2)$ :

Lemma 5.4. Assume $\left(h, g, F, \mathbb{B}_{T}\right)$ is as in Theorem 5.3. Then the envelope $U$ admits the following Taylor expansion at almost every point $\left(t_{0}, z_{0}\right) \in \mathbb{B}_{T}$,

$$
\begin{aligned}
U(t, z) & =U\left(t_{0}, z_{0}\right)+\left(t-t_{0}\right) \partial_{t} U\left(t_{0}, z_{0}\right)+\Re P\left(z-z_{0}\right)+L\left(z-z_{0}\right) \\
& +o\left(\left|t-t_{0}\right|+\left|z-z_{0}\right|^{2}\right),
\end{aligned}
$$

where $P$ is a polynomial of degree 2 and $L$ is the Levi form of $U\left(t_{0}, z\right)$ at $z_{0}$.

Proof. It follows from Theorem 4.7 that $U$ is locally uniformly semi-concave in $t \in] 0, T$. Theorem 5.3 ensures that $U$ is locally uniformly Lipschitz in $z \in \mathbb{B}$, hence, for all $t \in] 0, T[, U(t, \cdot)$ is twice differentiable at a.e. $z \in \mathbb{B}$.

Let $A_{1}$ be the set of points $\left(t_{0}, z_{0}\right) \in \Omega_{T}$ such that $U\left(\cdot, z_{0}\right)$ is not differentiable at $t_{0}$ and $A_{2}$ be the set of points $\left(t_{0}, z_{0}\right) \in \Omega_{T}$ such that $U\left(t_{0}, \cdot\right)$ is not twice differentiable at $z_{0}$. It follows from Fubini's Theorem that the set $A:=A_{1} \cup A_{2}$ is of Lebesgue measure zero in $\Omega_{T}$.

We show that the Taylor expansion holds at any point $\left(t_{0}, z_{0}\right) \notin A$. Fix $\varepsilon>0$ and $\left(t_{0}, z_{0}\right) \notin A$. We first write for $(t, z) \in \Omega_{T}$,

$$
U(t, z)-U\left(t_{0}, z_{0}\right)=U(t, z)-U\left(t_{0}, z\right)+U\left(t_{0}, z\right)-U\left(t_{0}, z_{0}\right) .
$$

Since $\left(t_{0}, z_{0}\right) \notin A_{1}$, the function $U\left(t_{0}, \cdot\right)$ is twice differentiable at $z_{0}$. Thus there exists $r>0$ such that for $\left|z-z_{0}\right|<r$,

$$
\left|U\left(t_{0}, z\right)-U\left(t_{0}, z_{0}\right)-\Re P\left(z-z_{0}\right)-L\left(z-z_{0}\right)\right| \leq \varepsilon\left|z-z_{0}\right|^{2} .
$$


On the other hand since $\left(t_{0}, z_{0}\right) \notin A_{2}, \partial_{t} U\left(t_{0}, z_{0}\right)$ exists and we have

$$
\begin{aligned}
U(t, z)-U\left(t_{0}, z\right)-\left(t-t_{0}\right) \partial_{t} U\left(t_{0}, z_{0}\right) & =\int_{t_{0}}^{t}\left(\partial_{\tau}^{+} U(\tau, z)-\partial_{t} U\left(t_{0}, z_{0}\right)\right) d \tau \\
& =\int_{t_{0}}^{t}\left(\partial_{\tau}^{-} U(\tau, z)-\partial_{t} U\left(t_{0}, z_{0}\right)\right) d \tau
\end{aligned}
$$

Since $\partial_{\tau}^{+} U$ is lsc and $\partial_{\tau}^{-} U$ is usc, we can choose $r$ so small that for $\left|t-t_{0}\right|+\left|z-z_{0}\right|<r$,

$$
\left|U(t, z)-U\left(t_{0}, z\right)-\left(t-t_{0}\right) \partial_{t} U\left(t_{0}, z_{0}\right)\right| \leq \varepsilon\left|t-t_{0}\right| .
$$

The Taylor expansion thus follows from (5.4) and (5.5).

\section{Pluripotential solutions}

We finally prove in this section that $U_{h, g, F, \Omega_{T}}$ is the unique pluripotential solution to the Cauchy-Dirichlet problem for (CMAF) which is locally uniformly semi-concave.

6.1. The case of Euclidean balls. We first treat the case when $\Omega$ is a euclidean ball in $\mathbb{C}^{n}$. By scaling and translating, it suffices to treat the case of the unit ball.

Theorem 6.1. Let $\Omega=\mathbb{B}$ be the unit ball in $\mathbb{C}^{n}, T<+\infty$, and assume that

(1) $G:=\log g$ is $\mathcal{C}^{1,1}$ in $\overline{\mathbb{B}}$;

(2) $h$ is uniformly $\mathcal{C}^{1,1}$ in $z \in \partial \mathbb{B}, h_{0}$ is $\mathcal{C}^{1,1}$ in $\overline{\mathbb{B}}$;

(3) $h$ is uniformly Lipschitz in $t \in\left[0, T\left[\right.\right.$ and $\partial_{t}^{2} h \leq C t^{-2}$ on $] 0, T[\times \partial \mathbb{B}$;

(4) $F$ is Lipschitz and semi-convex in $(t, z, r) \in[0, T[\times \overline{\mathbb{B}} \times J$ for each $J \Subset \mathbb{R}$.

Then for almost every $(t, z) \in \mathbb{B}_{T}$,

$$
\operatorname{det}\left(\frac{\partial^{2} U}{\partial z_{j} \partial \bar{z}_{k}}(t, z)\right)=e^{\dot{U}(t, z)+F(t, z, U(t, z))+G(z)} .
$$

In particular $U$ is a pluripotential solution to the Cauchy-Dirichlet problem for the parabolic equation (CMAF) with boundary data $h$.

Proof. Theorem 4.6 and the Lipschitz assumption on $h$ ensure that $U$ is a subsolution to (CMAF) with $U=h$ on $\partial_{0} \Omega_{T}$. It follows from Corollary 5.2 and Theorem 5.3 that $U$ is uniformly Lipschitz in $z \in \overline{\mathbb{B}}$ and locally $\mathcal{C}^{1,1}$ in $\mathbb{B}$. In particular $U$ is twice differentiable in $z$ almost everywhere in $\Omega_{T}$, hence

$$
\left(d d^{c} U\right)^{n}=\operatorname{det}\left(U_{j, \bar{k}}(t, z)\right) d V(z) .
$$

As $U$ is also almost everywhere differentiable in $t$ and a subsolution to the parabolic equation (CMAF), we infer by Proposition 3.2,

$$
\operatorname{det}\left(U_{j, \bar{k}}(t, z)\right) \geq e^{\partial_{t} U(t, z)+F(t, z, U(t, z))+G(z)},
$$

almost everywhere in $\mathbb{B}_{T}$. 
We want to prove that equality holds in (6.1). We use the notation of the proof of Lemma 5.4 and set $E=\mathbb{B}_{T} \backslash A$. Arguing by contradiction we assume that

$$
\operatorname{det}\left(U_{j, \bar{k}}\left(t_{0}, z_{0}\right)-\varepsilon I_{n}\right)>e^{\dot{U}\left(t_{0}, z_{0}\right)+F\left(t_{0}, z_{0}, U\left(t_{0}, z_{0}\right)\right)+G\left(z_{0}\right)+\varepsilon},
$$

at some point $\left(t_{0}, z_{0}\right) \in E$, for a small constant $\varepsilon>0$.

We use a bump construction to produce a subsolution $v \in \mathcal{S}_{h, g, F}\left(\mathbb{B}_{T}\right)$ which satisfies $v\left(t_{0}, z_{0}\right)>U\left(t_{0}, z_{0}\right)$ providing a contradiction. It follows from Lemma 5.4 that

$$
\begin{aligned}
U(t, z)-U\left(t_{0}, z_{0}\right) & =\left(t-t_{0}\right) \partial_{t} U\left(t_{0}, z_{0}\right)+\Re P\left(z-z_{0}\right)+L\left(z-z_{0}\right) \\
& +o\left(\left|t-t_{0}\right|+\left|z-z_{0}\right|^{2}\right) .
\end{aligned}
$$

Set $D_{r}:=\left\{(t, z) ;\left|t-t_{0}\right|+\left|z-z_{0}\right|^{2}<r\right\}$ and define

$$
\begin{aligned}
w(t, z) & :=U\left(t_{0}, z_{0}\right)+\partial_{t} U\left(t_{0}, z_{0}\right)\left(t-t_{0}\right)+\Re P\left(z-z_{0}\right) \\
& +L\left(z-z_{0}\right)+\delta-\gamma\left(\left|z-z_{0}\right|^{2}+\left|t-t_{0}\right|\right),
\end{aligned}
$$

where $\delta, \gamma>0$ are constants to be specified later. Note that if $\gamma$ is small enough then $w \in \mathcal{P}\left(D_{r}\right)$. For any $(t, z) \in D_{r}$, the Taylor expansion (6.2) ensures that

$$
\left.U(t, z) \geq w(t, z)+\gamma\left(\left|t-t_{0}\right|\right)+\left|z-z_{0}\right|^{2}\right)-\delta+o(r) .
$$

Hence for any $(t, z) \in D_{r} \backslash D_{r / 2}$,

$$
U(t, z) \geq w(t, z)+\gamma r / 2-\delta+o(r)>w(t, z),
$$

if $\delta=\gamma r / 4$, and $r>0$ is small enough. On the other hand for $(t, z) \in D_{r}$,

$$
\left(d d^{c} w\right)^{n}=\left(d d^{c} U-\gamma\left|z-z_{0}\right|^{2}\right)^{n}\left(t_{0}, z_{0}\right),
$$

and for $(t, z) \in D_{r}, t \neq t_{0}$,

$$
\partial_{t} w(t, z)=\partial_{t} U\left(t_{0}, z_{0}\right)-\gamma\left(t-t_{0}\right) /\left|t-t_{0}\right| .
$$

Thus if $\gamma<\varepsilon$, we obtain for any $(t, z) \in D_{r}$,

$$
\begin{aligned}
\left(d d^{c} w(t, z)\right)^{n} & \geq e^{\left.\left.\partial_{t} w(t, z)+\gamma\left(t-t_{0}\right) /\left|t-t_{0}\right|\right)\right)+F\left(t_{0}, z_{0}, U\left(t_{0}, z_{0}\right)\right)+G\left(z_{0}\right)+\varepsilon} d V \\
& \geq e^{\partial_{t} w(t, z)-\gamma+F(t, z, w(t, z))+G(z)+R(t, z)+\varepsilon} d V
\end{aligned}
$$

where

$$
R(t, z):=F\left(t_{0}, z_{0}, U\left(t_{0}, z_{0}\right)\right)-F(t, z, w(t, z))+\left(G\left(z_{0}\right)-G(z)\right) .
$$

Since $U$ and $F$ are locally Lipschitz, there exists $A>0$ such that for $r>0$ small enough and $(t, z) \in D_{r}$,

$$
R(t, z) \geq-A \sqrt{r} \geq \gamma-\varepsilon
$$

The function $w$ is therefore a subsolution to (CMAF) in $D_{r}$.

The previous estimates ensure that the function

$$
v(t, z):=\left\{\begin{array}{llr}
\max \{U(t, z), w(t, z)\} & \text { if } & (t, z) \in D_{r} \\
U(t, z) & \text { if } & (t, z) \in \mathbb{B}_{T} \backslash D_{r}
\end{array}\right.
$$


belongs to $\mathcal{S}_{h, g, F}\left(\mathbb{B}_{T}\right)$, hence $v \leq U$ in $\mathbb{B}_{T}$. In particular, $w \leq U$ in $D_{r}$ which is a contradiction since $w\left(t_{0}, z_{0}\right)=U\left(t_{0}, z_{0}\right)+\delta>U\left(t_{0}, z_{0}\right)$.

We now relax the regularity assumptions in Theorem 6.1.

Proposition 6.2. Assume $\Omega=\mathbb{B}$ is the unit ball in $\mathbb{C}^{n}, T<+\infty$, and

- $G:=\log g$ is continuous in $\overline{\mathbb{B}}$;

- $h$ is continuous on $\partial_{0} \mathbb{B}_{T}$ and satisfies (4.1) and (4.10);

- $F$ extends as a continuous function on $[0, T[\times \overline{\mathbb{B}} \times \mathbb{R}$ which is uniformly Lipschitz and uniformly semi-convex in $(t, r) \in[0, T[\times J$ for each $J \Subset \mathbb{R}$.

Then $U_{h, g, F, \mathbb{B}_{T}}$ is a continuous solution to (CMAF) with boundary values $h$.

Proof. It follows from Theorem 4.6 that $U \in \mathcal{S}_{h, g, F}\left(\mathbb{B}_{T}\right)$ satisfies the boundary conditions $(0.2),(0.3)$. It remains to prove that $U$ is continuous on $\left[0, T\left[\times \bar{\Omega}\right.\right.$ and solves $(\mathrm{CMAF})$ in $\Omega_{T}$. By Proposition 4.1 it suffices to prove these statements in $\mathbb{B}_{S}$ for each fixed $S<T$. We proceed in several steps.

Step 1. Assume that $h(\cdot, z)$ is uniformly Lipschitz in $t \in[0, T$. It follows from Theorem 5.1 that $U$ is continuous on $[0, T[\times \bar{\Omega}$. The goal is to prove that $U$ solves $(\mathrm{CMAF})$ in $\mathbb{B}_{S}$. We proceed by approximation as follows.

Let $\left(G_{j}\right)=\left(\log g_{j}\right)$ be a sequence of smooth functions uniformly converging to $G$ on $\overline{\mathbb{B}}$. Extending $F$ continuously in an open neighborhood of $[0, S] \times \overline{\mathbb{B}} \times \mathbb{R}$ and taking convolution in $(t, z, r)$ we can find a sequence $F_{j}:[0, S] \times \bar{B} \times \mathbb{R}$ of functions which are smooth in $(t, z, r)$ and

- Lipschitz and semi-convex in $[0, S] \times \overline{\mathbb{B}} \times J$ for each $J \Subset \mathbb{R}$;

- uniformly converges to $F$ on $[0, S] \times \overline{\mathbb{B}} \times J$, for each $J \Subset \mathbb{R}$.

We extend $h$ as a continuous function in $[0, T[\times\{|z| \geq 1 / 4\}$ by setting

$$
h(t, z):=h\left(t, \frac{z}{|z|}\right), z \in \mathbb{C}^{n},|z| \geq 1 / 4
$$

The extension $h$ satisfies (4.1) and (4.10) for all $|z| \geq 1 / 4$ (with the same constants $\kappa_{h}, C_{h}$ as the original function $h$ defined on $\partial_{0} \mathbb{B}_{T}$ ). Taking convolution in the $z$ variable we can find a sequence $\left(\hat{h}^{j}\right)$ of functions in $[0, T[\times\{|z|>1 / 3\}$ which are smooth in $z$ and

- are uniformly Lipschitz in $t$;

- satisfy (4.10) with the same uniform constant $C_{h}$;

- uniformly converge to $h$ on $[0, S] \times \partial \mathbb{B}$.

Fix $j \in \mathbb{N}$ and define $h^{j}$ by

$$
\left\{\begin{array}{cl}
h^{j}(t, z):=\hat{h}_{j}(t, z) & \text { if }(t, z) \in] 0, T[\times \partial \mathbb{B} \\
h^{j}(0, z)=h_{0}+H^{j} & \text { if }(t, z) \in\{0\} \times \mathbb{B}
\end{array}\right.
$$

where $H^{j}$ is the maximal plurisubharmonic function in $\mathbb{B}$ with boundary values $\hat{h}^{j}(0, \cdot)-h_{0}$. Observe that $h^{j}$ is a Cauchy-Dirichlet boundary data on $\mathbb{B}_{T}$ which satisfies the assumptions of Theorem 6.1. Note also that $h^{j}$ uniformly converges to $h$ on $\partial_{0} \mathbb{B}_{T}$, since $H^{j}$ uniformly converges to 0 . 
Set $U^{j}:=U_{h^{j}, g_{j}, F_{j}}\left(\mathbb{B}_{S}\right), j \in \mathbb{N}$. Theorem 6.1 ensures that $U^{j}$ is a pluripotential solution to the equation $(\mathrm{CMAF})$ and $U^{j}=h^{j}$ on $\partial_{0} \mathbb{B}_{S}$. It also follows from Theorem 4.2 and Theorem 4.7 that $U^{j}$ is locally uniformly semi-concave in $t \in] 0, S]$. Moreover, (4.5) and (4.13) ensure that the Lipschitz and semi-concave constants of $U^{j}$ are uniform. By definition of the envelope, $U^{j}$ uniformly converges to $U$ as $j \rightarrow+\infty$. It thus follows from Proposition 2.9, Proposition 2.3, Remark 2.4 and Lemma 2.8 that $U$ is a pluripotential solution to $(\mathrm{CMAF})$ in $\mathbb{B}_{S}$.

Step 2. To treat the general case, we approximate $h$ by a family of functions $h^{\varepsilon}$ which are Lipschitz up to zero, in such a way that they satisfy (4.1) and (4.10) with constants independent of $\varepsilon$.

We proceed as in the proof of Theorem 4.6. Fix $S>0$ and $\varepsilon>0$ such that $S+\varepsilon<T$, and define

$$
\begin{cases}h^{\varepsilon}(t, \zeta)=h(t+\varepsilon, \zeta) & \text { if }(t, \zeta) \in[0, S] \times \partial \mathbb{B} \\ h^{\varepsilon}(0, z)=h_{0}(z)+\phi_{\varepsilon}(z) & \text { if } z \in \mathbb{B}\end{cases}
$$

where $\phi_{\varepsilon}$ is the maximal plurisubharmonic function in $\mathbb{B}$ such that $\phi_{\varepsilon}(\zeta)=$ $h(\varepsilon, \zeta)-h_{0}(\zeta)$ on $\partial \mathbb{B}$. Then $h^{\varepsilon}$ uniformly converges to $h$ on $\partial_{0} \mathbb{B}_{S}$.

Observe that $h^{\varepsilon}$ is a Cauchy-Dirichlet boundary data satisfying (4.1) and (4.10) with constants independent of $\varepsilon$. By construction $h^{\varepsilon}$ is uniformly Lipschitz in $t \in[0, S]$. The previous step shows that $U^{\varepsilon}:=U_{h^{\varepsilon}, g, F, \mathbb{B}_{S}}$ is a continuous pluripotential solution to (CMAF) with boundary data $h^{\varepsilon}$. By Proposition $4.1, U^{\varepsilon}$ uniformly converges to $U$ on $\mathbb{B}_{S}$. The continuity of $U^{\varepsilon}$ ensures that $U$ is continuous in $\mathbb{B}_{S}$. Since $h_{0}$ is continuous, Theorem 3.12 ensures that $U$ is continuous in $[0, S[\times \overline{\mathbb{B}}$. It follows from Theorem 4.2 and Theorem 4.7 that the family $U^{\varepsilon}$ is locally uniformly semi-concave in $\left.t \in\right] 0, S[$ with constants independent of $\varepsilon$; see (4.3) and (4.11). Arguing as in the last part of Step 1 we conclude that $U$ solves $(\mathrm{CMAF})$ in $\mathbb{B}_{S}$.

6.2. The case of bounded strictly pseudoconvex domains. We now consider the case of a smooth bounded strictly pseudoconvex domain.

We first prove the existence result in a particular case.

Proposition 6.3. Assume $T<+\infty$, h satisfies (4.1) and (4.10). Then $U_{h, g, F}$ is a pluripotential solution to the Cauchy-Dirichlet problem for the parabolic equation (CMAF) in $\Omega_{T}$ with boundary conditions (0.2) and (0.3).

Proof. It follows from Theorem 4.2, Theorem 4.6, Theorem 4.7 that $U$ is locally uniformly semi-concave in $t \in] 0, T\left[, U \in \mathcal{S}_{h, g, F}\left(\Omega_{T}\right)\right.$ and it satisfies the boundary conditions $(0.2)$ and (0.3). It remains to verify that $U$ solves (CMAF). We proceed in several steps.

Step 1. We first assume that $h_{0}$ and $G:=\log g$ are continuous in $\bar{\Omega}$. Then $U$ is also continuous on $[0, T[\times \bar{\Omega}$ thanks to Theorem 5.1.

Let $B \Subset \Omega$ be a small ball and $h_{B}$ denote the restriction of $U$ on the parabolic boundary of $B_{T}$. The boundary data $h_{B}$ for the Cauchy-Dirichlet problem for (CMAF) satisfies the assumption of Proposition 6.2. Also, 
the restriction of $U$ on $[0, T[\times B$ is a continuous subsolution to the Cauchy Dirichlet problem (CMAF) in $B_{T}$ with boundary data $h_{B}$. It follows from Proposition 6.2 that $U_{B}:=U_{h_{B}, g, F, B_{T}}$ is a pluripotential solution to (CMAF) with boundary data $h_{B}$ and $U_{B} \geq U$ in $B_{T}$.

The function $V$, which is defined as $U_{B}$ in $B_{T}$ and $U$ in $\Omega_{T} \backslash B_{T}$, belongs to $\mathcal{S}_{h, g, F}\left(\Omega_{T}\right)$. Hence $V=U$ is a pluripotential solution to (CMAF).

Step 2. We next assume $h_{0}$ is continuous, but we merely assume $g \in L^{p}$.

Let $\left(g_{j}\right)$ be a sequence of strictly positive continuous functions in $\bar{\Omega}$ that converges to $g$ in $L^{p}(\Omega)$. Set $U^{j}:=U_{h, g_{j}, F}$ and $U:=U_{h, g, F}$. Since the $L^{p}$-norm of $g_{j}$ is uniformly bounded, Theorem 4.2 and Theorem 4.7 ensure that the functions $U^{j}$ are locally uniformly semi-concave (with constants independent of $j$ ). It thus follows from Proposition 1.14 that a subsequence of $U^{j}$, still denoted by $U^{j}$, converges almost everywhere in $\Omega_{T}$ to a function $V \in \mathcal{P}\left(\Omega_{T}\right)$. Lemma 1.8 ensures that $U_{t}^{j}$ converges in $L^{1}(\Omega)$ to $V_{t}$, for all $t \in] 0, T[$. By Proposition 2.9, for almost all $t \in] 0, T\left[, \partial_{t} U^{j}(t, \cdot)\right.$ converges pointwise to $\partial_{t} V(t, \cdot)$. Thus, for almost all $\left.t \in\right] 0, T[$,

$$
e^{\partial_{t} U^{j}(t, \cdot)+F\left(t, \cdot, U^{j}\right)} g_{j} \stackrel{L^{p}(\Omega)}{\longrightarrow} e^{\partial_{t} V(t, \cdot)+F(t, \cdot, V)} g .
$$

A result due to Kołodziej (see [Koł96, End of the proof of Theorem 3], see also [DK14, Theorem 2.8]) ensures that $U^{j}(t, \cdot)$ uniformly converges to $V(t, \cdot)$ and $\left(d d^{c} U^{j}(t, \cdot)\right)^{n}$ converges in the sense of positive measures to $\left(d d^{c} V(t, \cdot)\right)^{n}$. Thus $d t \wedge\left(d d^{c} U^{j}\right)^{n}$ weakly converges in $\Omega_{T}$ to $d t \wedge\left(d d^{c} V\right)^{n}$ (see the proof of Proposition 2.3). Hence $V$ solves (CMAF) in $\Omega_{T}$. Lemma 3.9 and Corollary 3.11 ensure that $\left.V^{*}\right|_{\partial_{0} \Omega_{T}} \leq h$. Thus $V \leq U$.

To prove that $U \leq V$ we now use a perturbation argument following an idea of Kołodziej [Kol96] (see also [GLZ18]). For each $j$ let $\theta_{j}$ be the unique continuous psh function in $\bar{\Omega}$, vanishing on $\partial \Omega$ such that $\left(d d^{c} \theta_{j}\right)^{n}=$ $\left|g_{j}-g\right| d V$. It follows from [Kol98] that

$$
\lim _{j \rightarrow+\infty} \sup _{\bar{\Omega}}\left|\theta_{j}\right|=0 .
$$

Fix $0<S<T, \varepsilon>0$ small enough and set, for $(t, z) \in \Omega_{S}$,

$$
W^{j}(t, z):=W^{j, \varepsilon}(t, z):=U(t+\varepsilon, z)-\delta(\varepsilon) t+C(\varepsilon) \theta_{j}(z),
$$

where $\delta(\varepsilon)>0, C(\varepsilon)>0$ are constants to be chosen in such a way that $\delta(\varepsilon) \rightarrow 0$ but $C(\varepsilon)$ may blow up as $\varepsilon \rightarrow 0$. The goal is to prove that $W^{j} \in \mathcal{S}_{h, g_{j}, F}\left(\Omega_{S}\right)$. It follows from Lemma 3.13 that $U_{t}$ uniformly converges on $\bar{\Omega}$ to $h_{0}$, ensuring that

$$
b(\varepsilon):=\sup _{\partial_{0} \Omega_{S}}|U(t+\varepsilon, z)-h(t, z)| \stackrel{\varepsilon \rightarrow 0}{\longrightarrow} 0 .
$$

A direct computation shows that

$$
\begin{aligned}
\left(d d^{c} W^{j}\right)^{n} & \geq\left(d d^{c} U(t+\varepsilon, \cdot)\right)^{n}+C(\varepsilon)^{n}\left(d d^{c} \theta_{j}\right)^{n} \\
& \geq e^{\partial_{t} U(t+\varepsilon, \cdot)+F(t+\varepsilon, \cdot, U(t+\varepsilon, \cdot))} g(z) d V+C(\varepsilon)^{n}\left|g-g_{j}\right| d V .
\end{aligned}
$$


By the Lipschitz condition (4.4) on $F$ we can write

$$
|F(t+\varepsilon, \cdot, U(t+\varepsilon, \cdot))-F(t, \cdot U(t+\varepsilon, \cdot))| \leq \varepsilon \kappa_{F} .
$$

Since $r \mapsto F(t, z, r)$ is increasing,

$$
F(t, \cdot, U(t+\varepsilon, \cdot)) \geq F\left(t, \cdot, W^{j}(t, \cdot)\right)-A \varepsilon,
$$

where $A>0$ depends on $\kappa_{F}, M_{U}$. We choose $\delta(\varepsilon):=b(\varepsilon)+A \varepsilon$. Then

$$
\begin{aligned}
\left(d d^{c} W^{j}\right)^{n} & \geq e^{\partial_{t} U(t+\varepsilon, \cdot)+F\left(t, \cdot, W^{j}(t, \cdot)\right)-A \varepsilon} g(z) d V+C(\varepsilon)^{n}\left|g-g_{j}\right| d V \\
& \geq e^{\partial_{t} W^{j}(t, \cdot)+F\left(t, \cdot, W^{j}(t, \cdot)\right)} g(z) d V+C(\varepsilon)^{n}\left|g-g_{j}\right| d V
\end{aligned}
$$

and $\left.W^{j}\right|_{\partial_{0} \Omega_{S}} \leq h$. We now choose

$$
C(\varepsilon):=\left(\sup _{\Omega_{S}} \exp \left\{\partial_{t} U(t+\varepsilon, z)+F(t, z, U(t+\varepsilon, z))\right\}\right)^{1 / n}<+\infty .
$$

Since $r \mapsto F(t, z, r)$ is increasing we obtain

$$
\begin{aligned}
\left(d d^{c} W^{j}\right)^{n} & \geq e^{\partial_{t} W^{j}(t, \cdot)+F\left(t, \cdot, W^{j}(t, \cdot)\right)} g d V+e^{\partial_{t} W^{j}(t, \cdot)+F\left(t, \cdot, W^{j}(t, \cdot)\right)}\left|g-g_{j}\right| d V \\
& \geq e^{\partial_{t} W^{j}(t, \cdot)+F\left(t, \cdot W^{j}(t, \cdot)\right)} g_{j} d V .
\end{aligned}
$$

Thus $W^{j} \in \mathcal{S}_{h, g_{j}, F}\left(\Omega_{S}\right)$. Together with Proposition 4.1 this yields

$$
W^{j, \varepsilon} \leq U_{h, g_{j}, F, \Omega_{T}}, \text { for all }(t, z) \in \Omega_{S} .
$$

In (6.3) we first let $j \rightarrow+\infty$ and then $\varepsilon \rightarrow 0$ to arrive at $U \leq V$. Hence $U=V$ is a pluripotential solution to the parabolic Monge-Ampère equation (CMAF) with boundary data $h$.

Step 3. We finally remove the continuity assumption on $h_{0}$. Using Lemma 2.11 we find a sequence $h^{j}$ of continuous Cauchy-Dirichlet boundary data for $\Omega_{T}$ such that $h^{j}=h$ on $\left[0, T\left[\times \partial \Omega\right.\right.$ and $h^{j}$ decreases pointwise to $h$. The previous step ensures that $U^{j}:=U\left(h^{j}, g, F\right)$ solves (CMAF). Theorem 4.2 and Theorem 4.7 provide uniform concavity constants for $U^{j}$. Since $h^{j}$ decreases to $h, U \leq U^{j}$ decreases to some $V \in \mathcal{P}\left(\Omega_{T}\right)$. We thus have $\left.V^{*}\right|_{\partial_{0} \Omega_{T}} \leq h$, and Proposition 2.9 and Proposition 2.3 reveal that $V$ solves (CMAF). Thus $V$ is a candidate defining $U$, hence $U=V$.

We are now ready to prove a general existence result. Here $T$ may take the value $+\infty$. We assume that, for each $0<S<T$, there exists a constant $C(S)>0$ such that for all $(t, z) \in] 0, S] \times \partial \Omega$,

$$
t\left|\partial_{t} h(t, z)\right| \leq C(S) ; t^{2} \partial_{t}^{2} h(t, z) \leq C(S) .
$$

Theorem 6.4. If $h$ satisfies (6.4) then $U:=U_{h, g, F}$ is a pluripotential solution to the Cauchy-Dirichlet problem for (CMAF) in $\Omega_{T}$ with boundary condition $h$. Moreover, $U$ is continuous in $] 0, T[\times \bar{\Omega}$ and locally uniformly semi-concave in $t \in] 0, T[$.

In particular, if $h_{0}$ is continuous on $\bar{\Omega}$ then $U$ is continuous on $[0, T[\times \bar{\Omega}$. 
Proof. For $S \in] 0, T\left[\right.$ we define $U^{S}:=U_{h, g, F, \Omega_{S}}$. Proposition 6.3 ensures that $U^{S}$ solves (CMAF) with $U^{S}=h$ on $\partial_{0} \Omega_{S}$. It follows from Proposition 4.1 that, for $0<S_{1}<S_{2}<T, U^{S_{1}}=U^{S_{2}}$ on $\Omega_{S_{1}}$. Letting $S \rightarrow T$ we obtain a function $V \in \mathcal{P}\left(\Omega_{T}\right)$ which solves (CMAF) and satisfies $V=h$ on $\partial_{0} \Omega_{T}$. Obviously $U \leq U^{S}$, for all $\left.S \in\right] 0, T$, hence $U \leq V$. But $V$ is also a candidate defining $U$, hence $V \leq U$. Therefore $V=U$ solves (CMAF) in $\Omega_{T}$. Moreover, by Theorem 4.2 and Theorem $4.7, U^{S}$ is locally uniformly Lipschitz and semiconcave in $t \in] 0, S$ [, hence so is $U$.

It follows from Proposition 3.2 and Remark 3.3 that

$$
\left(d d^{c} U_{t}\right)^{n}=e^{\partial_{t} U_{t}+F\left(t, \cdot, U_{t}\right)} g d V
$$

for almost every $t \in] 0, T$. Since $\partial_{t} U$ is locally bounded and $h_{t}$ is continuous on $\partial \Omega$ for all $t \in] 0, T\left[\right.$, [Kol98] ensures that $U_{t}$ is continuous on $\bar{\Omega}$ for almost all $t \in] 0, T$. Since $U$ is locally uniformly Lipschitz in $t$ we infer that $U$ is continuous in $] 0, T[\times \bar{\Omega}$.

If $h_{0}$ is continuous on $\bar{\Omega}$ then Theorem 4.6 and the continuity of $U(t, \cdot)$ (for each $t \in] 0, T$ [ fixed) ensure that $U$ is continuous on $[0, T[\times \bar{\Omega}$.

6.3. Uniqueness. We have proved in Section 6.2 the existence of a pluripotential solution to (CMAF) which is locally uniformly semi-concave in $t$. Our next goal is to prove that this is the unique such solution :

Theorem 6.5. Let $\Phi, \Psi \in \mathcal{P}\left(\Omega_{T}\right) \cap L^{\infty}\left(\Omega_{T}\right)$ with boundary data $h_{\Phi}, h_{\Psi}$. Assume that

(1) $\Psi$ is locally uniformly semi-concave in $t \in] 0, T[$;

(2) $\Phi$ is a subsolution while $\Psi$ is a supersolution to (CMAF) in $\Omega_{T}$;

(3) $h_{\Phi}$ satisfies (6.4).

Then $h_{\Phi} \leq h_{\Psi} \Longrightarrow \Phi \leq \Psi$.

Here $h_{\Phi}, h_{\Psi}$ are Cauchy Dirichlet boundary data in $\Omega_{T}$. In particular, $h_{\Psi}(t, \cdot)$ is continuous on $\partial \Omega$, and the supersolution property of $\Psi$ implies that $\Psi$ is continuous in $] 0, T[\times \bar{\Omega}$ (see Theorem 6.4 ).

An important consequence of this comparison principle is the following uniqueness result :

Corollary 6.6. Assume that $\Phi, \Psi \in \mathcal{P}\left(\Omega_{T}\right)$ are two pluripotential solutions to (CMAF) with boundary values $h$ satisfying (6.4). If $\Phi, \Psi$ are locally uniformly semi-concave in $t \in] 0, T\left[\right.$ then $\Phi=\Psi$ in $\Omega_{T}$.

Proof. Let $U:=U_{h, g, F, \Omega_{T}}$. Then Theorem 6.4 ensures that $U$ solves (CMAF) and $U, \Phi, \Psi$ are continuous on $] 0, T[\times \bar{\Omega}$. By definition, $\Phi, \Psi \leq U$. It follows from Theorem 6.5 that $U \leq \Phi, \Psi$, hence equality.

We first establish Theorem 6.5 under extra assumptions :

Lemma 6.7. With the same assumptions as in Theorem 6.5, assume moreover that $\Phi$ is $\mathcal{C}^{1}$ in $t$, continuous on $] 0, T[\times \bar{\Omega}$, and $\Psi$ is continuous on $\left[0, T\left[\times \bar{\Omega}\right.\right.$. Then $h_{\Phi} \leq h_{\Psi} \Longrightarrow \Phi \leq \Psi$. 
The first assumption (that $\Phi$ is $\mathcal{C}^{1}$ in $t$ ) means that $(t, z) \mapsto \partial_{t} \Phi(t, z)$ exists and it is continuous on $] 0, T[\times \Omega$.

Proof. We fix $S \in] 0, T[, \varepsilon>0$ small enough, and prove that

$$
\Phi \leq \Psi+2 \varepsilon t \text { in } \Omega_{S}
$$

The function

$$
[0, S] \times \bar{\Omega} \ni(t, z) \mapsto W(t, z):=\Phi(t, z)-\Psi(t, z)-2 \varepsilon t
$$

is upper semi-continuous and bounded. We are done if the maximum is attained on $\partial_{0} \Omega_{S}$. We thus assume that $\max W$ is reached at some point $\left.\left.\left(t_{0}, z_{0}\right) \in\right] 0, S\right] \times \Omega$. We want to prove that $W\left(t_{0}, z_{0}\right) \leq 0$. Assume, by contradiction that it is not the case. Then the set

$$
K:=\left\{z \in \Omega ; W\left(t_{0}, z\right)=W\left(t_{0}, z_{0}\right)\right\}
$$

is compact and the maximum principle ensures that

$$
\partial_{t} \Phi\left(t_{0}, z\right) \geq \partial_{t}^{-} \Psi\left(t_{0}, z\right)+2 \varepsilon, \text { for all } z \in K .
$$

Since $\Psi$ is locally uniformly semi-concave in $t \in] 0, T$ [ and continuous on $\left[0, T\left[\times \bar{\Omega}\right.\right.$, the left derivative $\partial_{t}^{-} \Psi(t, z)$ exists and it is upper semi-continuous in $] 0, T[\times \Omega$. Hence we can find $r>0$ so small that

$$
\partial_{t} \Phi\left(t_{0}, z\right) \geq \partial_{t}^{-} \Psi\left(t_{0}, z\right)+\varepsilon, \text { for all } z \in B,
$$

where $B=B_{r}:=\{z \in \Omega ; \operatorname{dist}(z, K)<r\}$.

Since $\Phi$ is a subsolution (which is $\mathcal{C}^{1}$ in $t$ ) while $\Psi$ is a supersolution to (CMAF), Proposition 3.2 and Remark 3.3 ensure that

$$
\left(d d^{c} \varphi\right)^{n} \geq e^{F\left(t_{0}, z, \varphi(z)\right)-F\left(t_{0}, z, \psi(z)\right)+\varepsilon}\left(d d^{c} \psi\right)^{n},
$$

setting $\varphi:=\Phi\left(t_{0}, \cdot\right), \psi:=\Psi\left(t_{0}, \cdot\right)$. Since $\varphi$ and $\psi$ are continuous in $\Omega, F$ is increasing in $r$, and $\varphi(z) \geq \psi(z)+2 \varepsilon t_{0}$ on $K$, up to shrinking $B$ we can assume that

$$
\left(d d^{c} \varphi\right)^{n} \geq e^{\varepsilon}\left(d d^{c} \psi\right)^{n} \text { in } B .
$$

Set now $\varphi_{r}:=\varphi+m_{r}$, where $m_{r}:=\min _{\partial B}(\psi-\varphi)$. Since $\psi \geq \varphi_{r}$ on $\partial B$, the comparison principle [BT76] yields

$$
\int_{\left\{\psi<\varphi_{r}\right\} \cap B} e^{\varepsilon}\left(d d^{c} \psi\right)^{n} \leq \int_{\left\{\psi<\varphi_{r}\right\} \cap B}\left(d d^{c} \varphi_{r}\right)^{n} \leq \int_{\left\{\psi<\varphi_{r}\right\} \cap B}\left(d d^{c} \psi\right)^{n} .
$$

Therefore $\left(d d^{c} \psi\right)^{n}$ does not charge the set $\left\{z \in B ; \psi(z)<\varphi_{r}(z)\right\}$ and the domination principle (see e.g. [GLZ18, Proposition 1.2]) yields $\varphi_{r} \leq \psi$ in $B$. In particular

$$
\varphi\left(z_{0}\right)-\psi\left(z_{0}\right)+\min _{\partial B}(\psi-\varphi)=\varphi_{r}\left(z_{0}\right)-\psi\left(z_{0}\right) \leq 0 .
$$

Since $K \cap \partial B=\emptyset$, we obtain, for all $z \in \partial B, W\left(t_{0}, z\right)<W\left(t_{0}, z_{0}\right)$ hence

$$
\varphi(z)-\psi(z)<\varphi\left(z_{0}\right)-\psi\left(z_{0}\right) \leq \max _{\partial B}(\varphi-\psi),
$$

a contradiction. Thus $\Phi \leq \Psi+2 \varepsilon t$ and we conclude by letting $\varepsilon \rightarrow 0$. 
We next establish an estimate for supersolutions to (CMAF).

Lemma 6.8. Assume $\Psi \in \mathcal{P}\left(\Omega_{T}\right)$ has boundary data $h_{\Psi}$. If $\Psi$ is a pluripotential supersolution to (CMAF) then for all $(t, z) \in \Omega_{T}$,

$$
\Psi(t, z) \geq h_{\Psi}(0, z)-c(t),
$$

where $c(t)>0$ satisfies $\lim _{t \rightarrow 0^{+}} c(t)=0$.

Proof. Fix $0<S<T$. For $s>0$ small enough we set

$$
\delta(s):=\sup \left\{\left|h_{\Psi}(\tau, z)-h_{\Psi}(t, z)\right| ; z \in \partial \Omega, t, \tau \in[0, S],|t-\tau| \leq s\right\} .
$$

Since $h_{\Psi}$ is continuous on $\left[0, T\left[\times \partial \Omega\right.\right.$, we have $\lim _{s \rightarrow 0^{+}} \delta(s)=0$.

Fix $s \in] 0,(T-S) / 2[$. We are going to prove that

$$
\Psi(s, z) \geq h_{\Psi}(0, z)-\delta(s)+s(\rho(z)-C)+n(s \log (s / T)-s),
$$

where $\rho$ is defined in (0.7) and $C$ is a uniform constant.

Fix $\varepsilon \in] 0, s]$ and let $h^{\varepsilon}$ denote the restriction of $(t, z) \mapsto \Psi(t+\varepsilon, z)$ on $\partial_{0} \Omega_{s}$. Then $h^{\varepsilon}$ is a continuous boundary data on $\Omega_{s}$. Set, for $(t, z) \in \Omega_{s}$,

$$
u^{\varepsilon}(t, z):=\Psi(\varepsilon, z)-\delta(s)+t\left(\rho(z)-C_{1}\right)+n(t \log (t / T)-t),
$$

where $C_{1}$ is a positive constant. By definition of $\delta(s)$ we have

$$
u^{\varepsilon}(t, z) \leq \Psi(t+\varepsilon, z)=h^{\varepsilon}(t, z) \text {, for all }(t, z) \in \partial_{0} \Omega_{s} .
$$

Arguing as in the proof of Lemma 3.8 we see that for $C_{1}>0$ big enough (depending on $M_{F}$ ), $u^{\varepsilon}$ is a pluripotential subsolution to (CMAF) in $\Omega_{s}$. Moreover, $u_{\varepsilon}$ is of class $\mathcal{C}^{1}$ in $t \in[0, s]$. On the other hand, a direct computation shows that, for $C_{2}>0$ large enough and under control (depending on $\kappa_{F}$ ), the function

$$
[0, s] \times \Omega \ni(t, z) \mapsto w^{\varepsilon}(t, z):=\Psi(t+\varepsilon, z)+C_{2} \varepsilon t
$$

is a pluripotential supersolution to (CMAF) and $w^{\varepsilon} \geq h^{\varepsilon}$ on $\partial_{0} \Omega_{s}$. By assumption on $\Psi, w^{\varepsilon}$ is continuous on $[0, s] \times \bar{\Omega}$. It thus follows from Lemma 6.7 that $w^{\varepsilon} \geq u^{\varepsilon}$ on $[0, s] \times \Omega$. We conclude by letting $\varepsilon \rightarrow 0$.

We next remove the continuity assumption on $\Psi$ in Lemma 6.7.

Lemma 6.9. With the same assumptions as in Theorem 6.5, assume moreover that $\Phi$ is $\mathcal{C}^{1}$ in $t$ and continuous on $] 0, T[\times \bar{\Omega}$. Then

$$
h_{\Phi} \leq h_{\Psi} \Longrightarrow \Phi \leq \Psi \text {. }
$$

Proof. Since $h_{\Psi}$ is continuous on $[0, T[\times \partial \Omega$, the proof of Theorem 6.4 shows that $\Psi$ is continuous in $] 0, T[\times \bar{\Omega}$, but it may not be continuous on $[0, T[\times \bar{\Omega}$. We use an idea in [DL17], exploiting the regularity of $\Psi$ at positive times close to zero. We fix $S \in] 0, T$ [ and prove that $\Phi \leq \Psi$ on $\Omega_{S}$.

Fix $s \in] 0,(T-S) / 2[$ and set, for $(t, z) \in[0, S] \times \bar{\Omega}$,

$$
v(t, z):=\Psi(t+s, z)+c(s)+\delta(s)+\text { Ast, }
$$

where $\delta(s)$ is defined in (6.5), $A>0$ is a constant, and $c(s)>0$ is as in Lemma 6.8 (which ensures $\Psi(s, z) \geq h_{\Psi}(0, z)-c(s)$ ). 
From the definition of $\delta(s)$ it follows that $v(t, z) \geq \Psi(t, z)=h_{\Psi}(t, z)$ on $[0, S] \times \partial \Omega$. For $A>0$ large enough (depending on $\kappa_{F}$ ), a direct computation shows that $v$ is a supersolution to (CMAF). Since $v$ is continuous on $[0, S] \times$ $\bar{\Omega}$, Lemma 6.7 then applies and yields $\Phi(t, z) \leq v(t, z)$ on $[0, S] \times \Omega$. We conclude by letting $s \rightarrow 0$.

We are now ready to prove the comparison principle.

Proof of Theorem 6.5. We can assume without loss of generality that $\Phi=$ $U_{h_{\Phi}, g, F}$. From assumption (3) and Theorem 6.4 we deduce that $U_{h_{\Phi}, g, F}$ is continuous on $] 0, T[\times \bar{\Omega}$. We would like to apply Lemma 6.9 but $\Phi$ is a priori not $\mathcal{C}^{1}$ in $t$. We are going to regularize $\Phi$ by taking convolution in $t$.

Fix $0<S<T$. For $s>0$ near 1 we set, for $(t, z) \in \Omega_{S}$,

$$
\left.W^{s}(t, z):=s^{-1} \Phi(s t), z\right)-C|s-1|(t+1) .
$$

If $C>0$ is large enough, the proof of Theorem 4.2 ensures that $W^{s} \in$ $\mathcal{S}_{h_{\Phi}, g, F}\left(\Omega_{S}\right)$. Let $\left\{\chi_{\varepsilon}\right\}_{\varepsilon>0}$ be a family of smoothing kernels in $\mathbb{R}$ approximating the Dirac mass $\delta_{0}$. For $\varepsilon>0$ small enough we define

$$
\Phi^{\varepsilon}(t, z):=\int_{\mathbb{R}} W^{s}(t, z) \chi_{\varepsilon}(s) d s .
$$

We are going to prove that $\Phi^{\varepsilon}$ (or $\Phi^{\varepsilon}-O(\varepsilon)$ ) is again a subsolution and use the previous step to conclude.

Let $\mathcal{H}$ denote the space of hermitian positive definite matrix $H$ that are normalized by $\operatorname{det} H=1$, and let $\Delta_{H}$ denote the Laplace operator

$$
\Delta_{H} \varphi:=\frac{1}{n} \sum_{j, k=1}^{n} h_{j k} \frac{\partial^{2} \varphi}{\partial z_{j} \partial \bar{z}_{k}} .
$$

Fix $H \in \mathcal{H}$. Since $W^{s} \in \mathcal{S}_{h_{\Phi}, g, F}\left(\Omega_{T}\right)$, Proposition 3.2 and [GLZ17, Main Theorem] yield

$$
\Delta_{H} W^{s}(t, z) \geq \exp \left(\frac{\partial_{t} W^{s}(t, z)+F\left(t, z, W^{s}(t, z)\right)}{n}\right) g(z)^{1 / n} .
$$

By definition of $\Phi^{\varepsilon}$ we obtain, using the convexity of the exponential,

$$
\begin{aligned}
& \Delta_{H} \Phi^{\varepsilon}(t, z)=\int_{\mathbb{R}} \Delta_{H} W^{s}(t, z) \chi_{\varepsilon}(s) d s \\
& \geq g(z)^{1 / n} \int_{\mathbb{R}} \exp \left(\frac{\partial_{t} W^{s}(t, z)+F\left(t, z, W^{s}(t, z)\right)}{n}\right) \chi_{\varepsilon}(s) d s \\
& \geq g(z)^{1 / n} \exp \left(\frac{1}{n}\left(\int_{\mathbb{R}}\left(\partial_{t} W^{s}(t, z)+F\left(t, z, W^{s}(t, z)\right)\right) \chi_{\varepsilon}(s) d s\right)\right) .
\end{aligned}
$$


Step 1. To simplify we first treat the case when $F$ is convex in $r$. Thus

$$
\begin{aligned}
& \Delta_{H} \Phi^{\varepsilon}(t, z)=\int_{\mathbb{R}} \Delta_{H} W^{s}(t, z) \chi_{\varepsilon}(s) d s \\
& \geq g(z)^{1 / n} \exp \left(\frac{1}{n}\left(\partial_{t} \Phi^{\varepsilon}(t, z)+F\left(t, z, \int_{\mathbb{R}} W^{s}(t, z) \chi_{\varepsilon}(s) d s\right)\right)\right) \\
& =g(z)^{1 / n} \exp \left(\frac{1}{n}\left(\partial_{t} \Phi^{\varepsilon}(t, z)+F\left(t, z, \Phi^{\varepsilon}(t, z)\right)\right)\right) .
\end{aligned}
$$

Using Proposition 3.2 and [GLZ17, Main Theorem] again, we infer that $\Phi^{\varepsilon}$ is a subsolution to (CMAF) in $\Omega_{S}$.

We now check that $\left(\Phi^{\varepsilon}\right)^{*}-O(\varepsilon) \leq h_{\Phi}$ on $\partial_{0} \Omega_{S}$. Indeed, for $z \in \partial \Omega$ we have $W^{s}(t, z) \leq h_{\Phi}(t, z)$, for all $s$, thus $\Phi^{\varepsilon}(t, z) \leq h_{\Phi}(t, z)$ for all $(t, z) \in$ $[0, S] \times \partial \Omega$. It remains to check that $\left(\Phi^{\varepsilon}\right)^{*}(0, z) \leq h_{\Phi}(0, z)$, for all $z \in \Omega$. It follows from Theorem 6.4 that $U_{h_{\Phi}, g, F, \Omega_{T}}$ has boundary value $h_{\Phi}$, hence, for $C$ large enough

$$
\lim _{t \rightarrow 0} W^{s}(t, z) \leq h_{\Phi}(0, z)-C|s-1|, \text { for all } z \in \bar{\Omega} .
$$

From the definition of $\Phi^{\varepsilon}$ in (6.6) it follows that

$$
\lim _{t \rightarrow 0} \Phi^{\varepsilon}(t, z) \leq h_{\Phi}(0, z), \forall z \in \bar{\Omega} .
$$

Hence $\Phi^{\varepsilon}-O(\varepsilon) t \in \mathcal{S}_{h_{\Phi}, g, F}\left(\Omega_{S}\right)$. Moreover, $\Phi^{\varepsilon}$ is of class $\mathcal{C}^{1}$ in $\left.t \in\right] 0, S[$ and $\Phi^{\varepsilon}$ converges pointwise to $\Phi$ as $\varepsilon \rightarrow 0$. Using Lemma 6.9 we obtain $\Phi^{\varepsilon} \leq \Psi$ in $\Omega_{S}$. The conclusion follows by letting $\varepsilon \rightarrow 0$.

Step 2. We now treat the case when $F$ is merely uniformly semi-convex in $r$. It follows from (4.2) and Theorem 4.2 that the functions $s \mapsto W^{s}(t, z)$, $(t, z) \in \Omega_{S}$, are uniformly Lipschitz in $[1 / 2,3 / 2]$. Thus for all $(t, z) \in \Omega_{S}$,

$$
\left|\Phi^{\varepsilon}(t, z)-\Phi(t, z)\right| \leq C \varepsilon
$$

for some uniform constant $C$, hence

$$
\int_{\mathbb{R}}\left(W^{s}\right)^{2}(t, z) \chi_{\varepsilon}(s) d s-\left(\int_{\mathbb{R}} W^{s}(t, z) \chi_{\varepsilon}(s) d s\right)^{2}=O(\varepsilon) .
$$

Recall (assumption (0.5)) that the function $r \mapsto F(t, z, r)+C_{F} r^{2}$ is convex in a large interval $J \Subset \mathbb{R}$, for fixed $(t, z) \in \Omega_{S}$. Jensen's inequality yields

$$
\begin{aligned}
& \int_{\mathbb{R}}\left(F\left(t, z, W^{s}(t, z)\right)+C_{F}\left(W^{s}(t, z)\right)^{2}\right) \chi_{\varepsilon}(s) d s \\
& \quad \geq F\left(t, z, \int_{\mathbb{R}} W^{s}(t, z) \chi_{\varepsilon}(s) d s\right)+C_{F}\left(\int_{\mathbb{R}} W^{s}(t, z) \chi_{\varepsilon}(s) d s\right)^{2} .
\end{aligned}
$$

Using this and (6.7) we obtain

$$
\int_{\mathbb{R}} F\left(t, z, W^{s}(t, z)\right) \chi_{\varepsilon}(s) d s-F\left(t, z, \int_{\mathbb{R}} W^{s}(t, z) \chi_{\varepsilon}(s) d s\right) \geq O(\varepsilon) .
$$

We repeat the previous step to conclude that $\Phi^{\varepsilon}-O(\varepsilon) t \in \mathcal{S}_{h_{\Phi}, g, F}\left(\Omega_{S}\right)$. 


\section{REFERENCES}

[BT76] E. Bedford and B. A. Taylor, The Dirichlet problem for a complex Monge-Ampère equation, Invent. Math. 37 (1976), no. 1, 1-44.

[BT82] E. Bedford and B. A. Taylor, A new capacity for plurisubharmonic functions, Acta Math. 149 (1982), no. 1-2, 1-40.

[Bło05] Z. Błocki, Weak solutions to the complex Hessian equation, Ann. Inst. Fourier, Grenoble 55, 5 (2005), 1735-1756.

[CT15] T. Collins, V. Tosatti, Kähler currents and null loci. Invent. Math. 202 (2015), no. 3, 1167-1198.

[Dem91] J. P. Demailly, Potential Theory in Several Complex Variables. Course available on the author's webpage.

[Din09] S. Dinew, An inequality for mixed monge-ampere measures, Mathematische Zeitschrift 262 (2009), no. 1, 1-15.

[DK14] S. Dinew, S. Kołodziej, A priori estimates for complex Hessian equations. Anal. PDE 7 (2014), no. 1, 227-244.

[DL17] E. Di Nezza and C. H. Lu, Uniqueness and short time regularity of the weak Kähler-Ricci flow, Adv. Math. 305 (2017), 953-993.

[Do17] Hoang-Son Do, Weak solution of Parabolic complex Monge-Ampère equation, Indiana Univ. Math. J. 66 (2017), no. 6, 1949-1979.

[Do16] Hoang-Son Do, Weak solution of parabolic complex Monge-Ampère equation II, Internat. J. Math. 27 (2016), no. 12, 1650098.

[EGZ11] P. Eyssidieux, V. Guedj, A. Zeriahi, Viscosity solutions to Degenerate Complex Monge-Ampère Equations. Comm. Pure Appl. Math. 64 (2011), no. 8, 1059-1094.

[EGZ15] P. Eyssidieux, V. Guedj, and A. Zeriahi, Weak solutions to degenerate complex Monge-Ampère flows I, Math. Ann. 362 (2015), no. 3-4, 931-963.

[EGZ18] P. Eyssidieux, V. Guedj, A. Zeriahi, Convergence of weak Kähler-Ricci flows on minimal models of positive Kodaira dimension. C.M.P. 357 (2018), no. 3, 1179-1214.

[GT01] D. Gilbarg, N.S. Trudinger, Elliptic Partial Differential Equations of Second Order, Springer-Verlag Berlin Heidelberg, Edition 2, pages XIII, 518.

[GLZ17] V. Guedj, C. H. Lu, A. Zeriahi, Weak subsolutions to complex Monge-Ampère equations, arXiv:1703.06728 (2017). Journal of the Math. Soc. Japan. to appear.

[GLZ18] V. Guedj, C. H. Lu, A. Zeriahi, Stability of solutions to complex Monge-Ampère flows, Ann. Inst. Fourier, to appear.

[GLZ2] V. Guedj, C. H. Lu, A. Zeriahi, Pluripotential Kähler-Ricci flows, preprint (2018).

[GLZ3] V. Guedj, C.H .Lu, A. Zeriahi, Viscosity vs Pluripotential solutions to complex Monge-Ampère flows. In preparation.

[GZ] V. Guedj, A. Zeriahi, Degenerate complex Monge-Ampère equations, EMS Tracts in Mathematics, vol. 26, European Mathematical Society (EMS), Zürich, 2017.

[Ham82] R.Hamilton, Three-manifolds with positive Ricci curvature. J. Diff. Geom. 17 (2) (1982), 255-306.

[HL11] F. R. Harvey, H. B. Lawson Jr, The Dirichlet duality and the nonlinear Dirichlet problem on Riemannian manifolds, J. Diff. Geom. 88 no. 3 (2011), 395-482.

[HL13] F. R. Harvey, H. B. Lawson Jr, The equivalence of viscosity and distributional subsolutions for convex subequations - a strong Bellman principle. Bull. Brazilian Math. Soc. 44 no. 4 (2013), 621-652.

[Hörm] L. Hörmander, Notions of convexity, Modern Birkhäuser Boston, Inc., Boston, MA, 2007, Reprint of the 1994 edition.

[Klim] M. Klimek, Pluripotential theory, London Mathematical Society Monographs. New Series, vol. 6, The Clarendon Press, Oxford University Press, New York, 1991.

[Koł95] S. Kołodziej, The range of the complex Monge-Ampère operator. II, Indiana Univ. Math. J. 44 (1995), no. 3, 765-782. 
[Kol96] S. Kołodziej, Some sufficient conditions for solvability of the Dirichlet problem for the complex Monge-Ampère operator. Ann. Polon. Math. 65 (1996), no. 1, 11-21.

[Koł98] S. Kołodziej, The complex Monge-Ampère equation, Acta Math. 180 (1998), no. 1, 69-117.

[Koł03] S. Kołodziej, The Monge-Ampère equation on compact Kähler manifolds, Indiana Univ. Math. J. 52 (2003), no. 3, 667-686.

[ST17] J. Song and G. Tian, The Kähler-Ricci flow through singularities, Invent. Math. 207 (2017), no. 2, 519-595.

[SW13] J. Song, B.Weinkove, Lecture notes on the Kähler-Ricci flow. "Introduction to the Kähler-Ricci flow", eds S. Boucksom, P. Eyssidieux, V. Guedj, L.N.M. 2086 (2013).

[Wal69] J. B. Walsh, Continuity of envelopes of plurisubharmonic functions, J. Math. Mech. 18 (1968/1969), 143-148.

[Yau78] S.-T. Yau, On the Ricci curvature of a compact Kähler manifold and the complex Monge-Ampère equation. I, Comm. Pure Appl. Math. 31 (1978), no. 3, 339-411.

Vincent Guedu, Institut de Mathématiques de Toulouse, Université de Toulouse, CNRS, UPS, 118 route de Narbonne, 31062 Toulouse cedex 09, France

E-mail address: vincent.guedj@math.univ-toulouse.fr

Hoang-Chinh Lu, Laboratoire de Mathématiques d'Orsay, Univ. Paris-Sud, CNRS, Université PARIS-SAClay, 91405 Orsay, France

E-mail address: hoang-chinh.lu@math.u-psud.fr

Ahmed Zeriahi, Institut de Mathématiques de Toulouse,, Université de Toulouse, CNRS, UPS, 118 route de Narbonne, 31062 Toulouse cedex 09, France

E-mail address: ahmed.zeriahi@math.univ-toulouse.fr 